\title{
SIGNATURES OF A COMPANION STAR IN TYPE IA SUPERNOVAE
}

AUTHOR(S):

Maeda, Keiichi; Kutsuna, Masamichi; Shigeyama, Toshikazu

\section{CITATION:}

Maeda, Keiichi ... [et al]. SIGNATURES OF A COMPANION STAR IN TYPE IA SUPERNOVAE. The Astrophysical Journal 2014, 794(1): 37.

\section{ISSUE DATE:}

2014-10-10

URL:

http://hdl.handle.net/2433/191103

RIGHT:

(C) 2014. The American Astronomical Society. 


\title{
SIGNATURES OF A COMPANION STAR IN TYPE IA SUPERNOVAE
}

\author{
Keitchi Maeda ${ }^{1,2}$, Masamichi Kutsuna ${ }^{3}$, and Toshikazu Shigeyama ${ }^{3}$ \\ ${ }^{1}$ Department of Astronomy, Kyoto University, Kitashirakawa-Oiwake-cho, Sakyo-ku, Kyoto 606-8502, Japan; keiichi.maeda@ kusastro.kyoto-u.ac.jp \\ ${ }^{2}$ Kavli Institute for the Physics and Mathematics of the Universe (WPI), Todai Institutes for Advanced Study, \\ University of Tokyo, 5-1-5 Kashiwanoha, Kashiwa, Chiba 277-8583, Japan \\ ${ }^{3}$ Research Center for the Early Universe, School of Science, University of Tokyo, 7-3-1 Hongo, Bunkyo-ku, Tokyo 113-0033, Japan \\ Received 2013 December 26; accepted 2014 August 15; published 2014 September 22
}

\begin{abstract}
Although type Ia supernovae (SNe Ia) have been used as precise cosmological distance indicators, their progenitor systems remain unresolved. One of the key questions is whether there is a nondegenerate companion star at the time of a thermonuclear explosion of a white dwarf. In this paper, we investigate whether an interaction between the SN ejecta and the companion star may result in observable footprints around the maximum brightness and thereafter, by performing multidimensional radiation transfer simulations based on hydrodynamic simulations of the interaction. We find that such systems result in variations in various observational characteristics due to different viewing directions, and the predicted behaviors (redder and fainter for the companion direction) are the opposite of what were suggested by the previous study. The variations are generally modest and within observed scatters. However, the model predicts trends between some observables different from those observationally derived, so a large sample of SNe Ia with small calibration errors may be used to constrain the existence of such a companion star. The variations in different colors in optical band passes can be mimicked by external extinctions, so such an effect could be a source of scatter in the peak luminosity and derived distance. After the peak, hydrogen-rich materials expelled from the companion will manifest themselves in hydrogen lines, but $\mathrm{H} \alpha$ is extremely difficult to identify. Alternatively, we find that $\mathrm{P}_{\beta}$ in postmaximum near-infrared spectra can potentially provide a powerful diagnostic.
\end{abstract}

Key words: distance scale - radiative transfer - supernovae: general

Online-only material: color figures

\section{INTRODUCTION}

Type Ia supernovae ( $\mathrm{SNe}$ Ia) are mature standardized candles and have been playing a key role in observational cosmology (Riess et al. 1998; Permutter et al. 1999). The SN Ia cosmology relies on an empirically derived relation between the peak luminosity and the light curve decline rate (e.g., $\Delta m_{15}$, defined as a magnitude decrease from the peak to 15 days after), the so-called Phillips relation (Phillips et al. 1999). This is also complemented by further relations between the luminosity (or decline rate) and the intrinsic colors (most frequently $B-V$ ) as essential in calibrating the external extinction (e.g., Folatelli et al. 2010 and references therein).

However, the progenitors and explosion of $\mathrm{SNe} \mathrm{Ia}$ are not yet fully understood. There has been a long debate about the progenitor systems. The proposed systems are largely divided into two categories. One is called the single degenerate (SD) scenario, where a white dwarf (WD) accretes materials from its binary nondegenerate companion star, either a red giant (RG) or a main sequence (MS), to increase its mass (nearly) to the Chandrasekhar limit and ignites carbon near the center (e.g., Whelan \& Iben 1973; Nomoto 1982; Hachisu et al. 1999). The companion stars could even be of different types (e.g., Wang et al. 2009; Wheeler 2012; Liu et al. 2013), but in this paper we mainly focus on RG and MS cases. The other scenario involves a merger of two WDs and is called the double-degenerate (DD) scenario (e.g., Iben \& Tutukov 1984; Webbink 1984). The merger of WDs may result in a prompt explosion (Pakmor et al. 2010), or it may resemble the final stage of the SD scenario with a larger accretion rate: a WD with a (nearly) Chandrasekhar mass may evolve hydrostatically toward the central ignition (Yoon et al. 2007), or such a system may lead to an accretioninduced collapse rather than SNe Ia (Saio \& Nomoto 1985).
Different scenarios will lead to different explosion mechanisms, and thus understanding the origin of the diversity and relations in SN Ia luminosity and other observational properties relies on understanding the progenitor and evolution scenarios. Different scenarios may well predict a different evolution of SN properties as a function of the redshift, so this is also a critical question in the SN Ia cosmology.

New developments on this issue have been achieved in the last few years from observational viewpoints. So far, the strongest constraints have been placed by direct searches for surviving nondegenerate companion stars. A supernova remnant (SNR) 0509-67.5 in the Large Magellanic Cloud (LMC) was found to have no point sources down to $M_{V} \sim 8.4$ in the central region, ruling out both RG and MS surviving companions for this particular SN (Schaefer \& Pagnotta 2010). It has been reported that a giant companion star is absent in SNR 1006 (González Hernández et al. 2012) and SN 2011fe in M101 (Li et al. 2011).

Somewhat model-dependent but still useful constraints on a possible companion star have been inferred from properties of nearby SNe Ia as well. Thermal energy deposited by a collision between the $\mathrm{SN}$ ejecta and the companion is predicted to produce a detectable blue emission in the premaximum rising phase, especially for the RG companion case (Kasen 2010), but such an effect has not been clearly seen in a large sample of SNe Ia (Hyden et al. 2010). In addition to the sign in the light curve, this collision is suggested to leave its sign in the spectrum. The hydrogen-rich envelope of the companion star is expected to be stripped away by the collision, being embedded mostly in the innermost, low-velocity part of the SN ejecta (Marietta et al. 2000). Such hydrogen-rich material is suggested to produce $\mathrm{H}_{\alpha}$ emission in the late phase (Mattila et al. 2005), but so far there has been no sign of $\mathrm{H}_{\alpha}$ in nearby $\mathrm{SNe}$ Ia (Mattila 
et al. 2005; Leonard 2007; Shappee et al. 2013; Lundqvist et al. 2013).

However, there are also observational indications for the SD scenario for at least some $\mathrm{SNe}$ Ia. There is a strong candidate for a surviving G-type dwarf in Tycho's SNR (Ruiz-Lapuente et al. 2004; Bedin et al. 2014) (but see also Ihara et al. 2007; Kerzendorf et al. 2009). The discovery of strongly interacting $\mathrm{SNe}$ Ia (SNe Ia exploding within dense circumstellar matter (CSM)) favors the SD scenario for these SNe, specifically systems in which the companion is still in the nondegenerate phase at the time of the explosion (Hamuy et al. 2003; Aldering et al. 2006; Dilday et al. 2012). In particular, Dilday et al. (2012) discovered evidence for the traces of nova explosions preceding the SN, which had been predicted by the SD scenario. From this we expect that there are also cases where a WD with a nondegenerate companion explodes but without showing strong CSM interaction signals (Hachisu et al. 2012). In sum, the issue is still controversial, and further study is required. In particular, the arguments based on SN properties can be model dependent (e.g., see Kutsuna \& Shigeyama 2013; Kutsuna 2013, for uncertainties of the collision-induced emission in the premaximum stage), and thus different ideas based on different physical processes and different observational strategies are quite useful. In this respect, we investigate the issue of how the maximum and postmaximum phases are affected by the existence of a companion star. While these are the most easily accessible observations, indeed the model predictions so far are mostly restricted to the early rising phase (a few days after the explosion) or the late-time nebular phase (about an year after the explosion). There has been only one study on this issue, by Kasen et al. (2004). They predicted that the maximum spectrum is generally not sensitive to the viewing angle, whereas the SN looks blue and peculiar (i.e., 1991Tlike) when viewed from the direction of the hole created by the ejecta-companion interaction. There were however some limitations in their study: (1) it has not been clarified whether the overall (or angle-averaged) properties are affected as compared to the noninteraction case; (2) the prediction was made only for the maximum spectra, so there were no specific features predicted for the postmaximum spectra, spectral evolution, or the multiband light curves; and finally, (3) the model was based on a toy model, which might be missing some ingredients important in hydrodynamics.

In this paper, we explore the maximum and postmaximum properties of SNe in the optical through near-infrared (NIR) wavelength ranges as a result of interaction between ejecta and a nondegenerate companion star. In Section 2, we summarize our hydrodynamic models and methods for radiation transfer simulations. In Section 3, we discuss overall properties in spectra and multiband light curves. In Section 4, we discuss details on individual spectral features, colors, and their mutual relations predicted by the simulations. In Section 5, we investigate a possibility to detect hydrogen lines as diagnostics of the nondegenerate companion star. The paper is closed in Section 6 with conclusions and discussion. We describe details of the radiation transfer simulation methodology in the appendixes.

\section{METHOD AND MODELS}

\subsection{Hydrodynamic Models}

Our input models for the radiation transfer simulations are taken from Kutsuna \& Shigeyama (2013; see also Kutsuna 2013). In this section, we summarize the main features of the

\begin{tabular}{lccc}
\multicolumn{3}{c}{$\begin{array}{c}\text { Table 1 } \\
\text { Models }\end{array}$} \\
\hline \hline Model & $\begin{array}{c}M_{2}{ }^{\mathrm{a}} \\
\left(M_{\odot}\right)\end{array}$ & $R_{2}\left(10^{13} \mathrm{~cm}\right)^{\mathrm{b}}$ & $A\left(10^{13} \mathrm{~cm}\right)^{\mathrm{c}}$ \\
\hline MS & 1 & 0.01 & 0.03 \\
RGa & 1 & 0.7 & 2 \\
RGb & 1 & 1 & 3 \\
\hline
\end{tabular}

Notes.

${ }^{a}$ The mass of the companion star.

${ }^{b}$ The radius of the companion star.

${ }^{\mathrm{c}}$ The binary separation.

models, and we refer to Kutsuna \& Shigeyama (2013) for further details of the models.

These models are results of radiation hydrodynamic simulations of the collision between the expanding SN ejecta and a nondegenerate companion star. Thus, the initial configuration is specified by a few binary parameters, namely the type of the companion star and the separation between the WD and the companion. We take three models from their simulations, named Models MS, RGa, and RGb. Basic features of these models are summarized in Table 1 . Model RGa represents a close binary system with an RG companion. The RG has $0.4 M_{\odot}$ of the He core and $0.6 M_{\odot}$ of the convective H-rich envelope. The separation is set to be $2 \times 10^{13} \mathrm{~cm}$. Model RGb is the same as RGa except for the separation being $3 \times 10^{13} \mathrm{~cm}$. Model MS represents a close binary system with an MS companion. The companion MS mass is $1 M_{\odot}$ and the separation is $3 \times 10^{11} \mathrm{~cm}$. The composition in the $\mathrm{H}$ envelope is set as $75 \%$ in $\mathrm{H}$ and $25 \%$ in He. We ignore the metal content in the hydrogen envelope (see Section 6). The radius of the companion is determined by a requirement that the companion star filled the Roche lobe just before the explosion.

Figure 1 shows the ejecta structure of Model RGa in the homologous expansion phase. The other models in the same phase are qualitatively similar to Model RGa, with only slight differences in details (e.g., in the opening angle of the hole created by the interaction). For details of the hydrodynamic behaviors, see Kutsuna \& Shigeyama (2013). The H-rich envelope mass stripped by the interaction is $\sim 0.4 M_{\odot}$ in all three models. We note here that while the hydrodynamic behaviors are generally consistent with previous studies (e.g., Marietta et al. 2000), the companion star in Model MS suffers from a large amount of hydrogen stripping. This is likely due to an insufficient computational resolution (Pakmor et al. 2008), so the amount of hydrogen in Model MS should be regarded as being overestimated (Kutsuna \& Shigeyama 2013; Kutsuna 2013). We note however that how much hydrogen is stripped away in the WD-MS system is still under debate (Liu et al. 2012).

The W7 model (Nomoto et al. 1984) was used for the SN ejecta model. Accidentally, in Kutsuna \& Shigeyama (2013; see also Kutsuna 2013), the innermost stable Ni was counted as radioactive ${ }^{56} \mathrm{Ni}$ in preparing the input model, so this model has $\sim 0.8 M_{\odot}$ of ${ }^{56} \mathrm{Ni}$, which is larger than in the original $\mathrm{W} 7$ model. After mapping onto our numerical grids for the radiation transfer, the amount of ${ }^{56} \mathrm{Ni}$ is $0.81 M_{\odot}$ in our ejecta model and $0.59 M_{\odot}$ in the original $\mathrm{W} 7$ model. In any case, because the exact explosion mechanism is not yet clarified and $M\left({ }^{56} \mathrm{Ni}\right)$ is within the observationally derived range of Branch-normal $\mathrm{SNe}$ Ia (Branch et al. 2006), we take this modified W7 model as our reference model. Note that we are mainly aiming at investigating differences between the SN Ia with and without a nondegenerate 

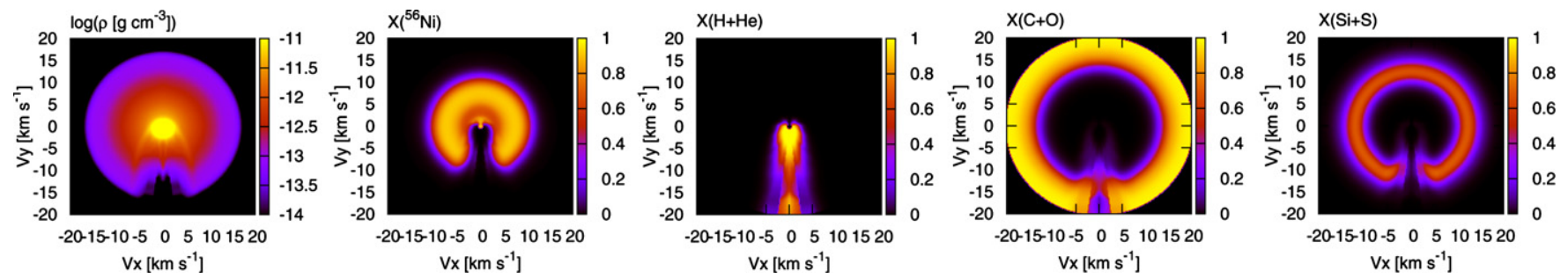

Figure 1. Ejecta structure of Model RGa. The density is scaled to the value at 10 days after the explosion (when the ejecta are already in a homologous expansion), The companion was initially on the $-y$ direction (i.e., toward the bottom). The viewing angle $\theta$ is defined to be $\theta=0$ in the companion direction ( $-y$ in this figure), and $\theta=\pi$ in the opposite direction $(+y)$.

(A color version of this figure is available in the online journal.)

companion and variations from different viewing directions, so details of the reference model are not important.

In Kutsuna \& Shigeyama (2013), the collision between the SN ejecta and the companion star was simulated by twodimensional radiation hydrodynamic simulations, with a few simplifications in the radiation transfer scheme (e.g., gray transfer, ignoring the bound-bound transition, a simplified $\gamma$-ray deposition scheme, flux-limited diffusion approximation). After the collision, the expanding ejecta (affected by the impact to the companion) reach the homologous expansion quickly, and the density structure in the homologous phase is little affected by the radiation transfer effect. Thus we adopt the density and composition structures at 35 days after the explosion as our reference and set the ejecta structure according to the homologous expansion to the initial time for the detailed radiation transfer simulations (typically 10 days after the explosion). Although the impact dissipates the kinetic energy (Kasen 2010; Kutsuna \& Shigeyama 2013), the resultant thermal energy is lost in a few days mostly due to adiabatic loss. As such, in the few days after the explosion, the thermal condition is determined by the radioactive input. Thus, we neglect the thermal energy content that is due to the impact in our radiation simulations.

In addition to these companion-interaction models, we also perform the radiation transfer simulations for the original W7 model and our own one-dimensional (1D) reference model without interaction. The reference 1D model is constructed as follows. We extract the radial information from Model MS in the direction opposite from the companion, and this radial structure is mapped into all of the directions in three-dimensional (3D) space. This model represents the SN ejecta model without the interaction. This model is used for a fair comparison to the interaction models, better than the original W7 model. The reasons for this are (1) the SN ejecta models used for the interaction simulations have the distribution and mass of ${ }^{56} \mathrm{Ni}$ slightly different from the original W7 model (see above), and (2) this is computed through the same hydrodynamic code with the interaction models, so a possible numerical diffusion is taken into account in this reference model in the same manner as in the interaction models.

\subsection{Radiation Transfer}

We have performed radiation transfer simulations for the input models described in Section 2.1, mapped onto the twodimensional (2D) axisymmetric coordinates with grid points $\left(n_{r}, n_{\theta}\right)=(50,50)$, where $r$ and $\theta$ represent radial and polar angle coordinates. This spatial resolution is enough to resolve the major features in the ejecta structure arising from the interaction (Figure 1) and also sufficient to resolve the spectral features arising from the photon Doppler shift (i.e., the radial spatial resolution corresponds to the photon Doppler shift $\sim 300 \mathrm{~km} \mathrm{~s}^{-1}$ finer than the typical spectral resolution in observations). We used a multidimensional/frequency/epoch radiation transfer code developed by us: HEIMDALL (Handling Emission In Multi-Dimension for spectrAL and Light curve calculations). The full details of the code are presented in Appendix A, and in this section we will provide a summary of the simulation method and description specific to simulations in this paper.

The code largely adopts the prescriptions presented by Lucy (2005), Kasen et al. (2006), and Kromer \& Sim (2009). The code solves radiation transfer for density and composition (taking into account radioactive decays) structures as a function of time given as an input model $\left[\rho(\boldsymbol{r}, t), X_{i}(\boldsymbol{r}, t)\right]$. The radiation field is solved with the Monte Carlo (MC) method, where the radiation field is discretized into photon packets and the interactions between the radiation and matter are treated as individual microscopic events in the comoving frame (Lucy 2005 and references therein). To solve the radiation transfer, a mixedframe approach is adopted, where the transformation from the comoving to the rest frames, and vice versa, automatically takes into account the Doppler shift of radiation with respect to the matter. This is essential in the $\mathrm{SN}$ radiation transfer because the large velocity gradient results in the wavelength shift of photons in the comoving frame even without interaction, and this wavelength shift can be much larger than the typical separations of the bound-bound transitions in the frequency space. The temperature at each position and time $[T(\boldsymbol{r}, t)]$ is iteratively solved with a radiation field (both in optical-NIR and $\gamma$-rays) within a time step, under the assumption of radiative equilibrium. Ionization and level populations are computed under the assumption of local thermodynamic equilibrium (LTE). The new temperature is then used for an initial guess of the temperature in the next time step, and the radiation field at the end of a given time step is used as the initial radiation field at the beginning of the next time step.

At the beginning of the simulation, MC packets representing $\gamma$-rays are created. They are assigned the frequency, energy, spatial position, and emission epoch, following the radioactive decay chain of ${ }^{56} \mathrm{Ni} \rightarrow{ }^{56} \mathrm{Co} \rightarrow{ }^{56} \mathrm{Fe}$. The transfer of $\gamma$-rays is then followed with the prescriptions given by Maeda (2006). The interactions include Compton scattering, photoelectric absorption, and pair creation. As a result, the energy deposition rate by $\gamma$-rays is obtained as a function of position and time. Together with the positron energy input, which is assumed to take place in situ, the heating/creation rate of optical (or thermal) photons is obtained.

Using the energy deposition rate obtained through the $\gamma$-ray transfer, thermal photon packets are created. These are then followed by the MC simulation as described above. For the opacity to thermal (UV through NIR) photons, we adopt 

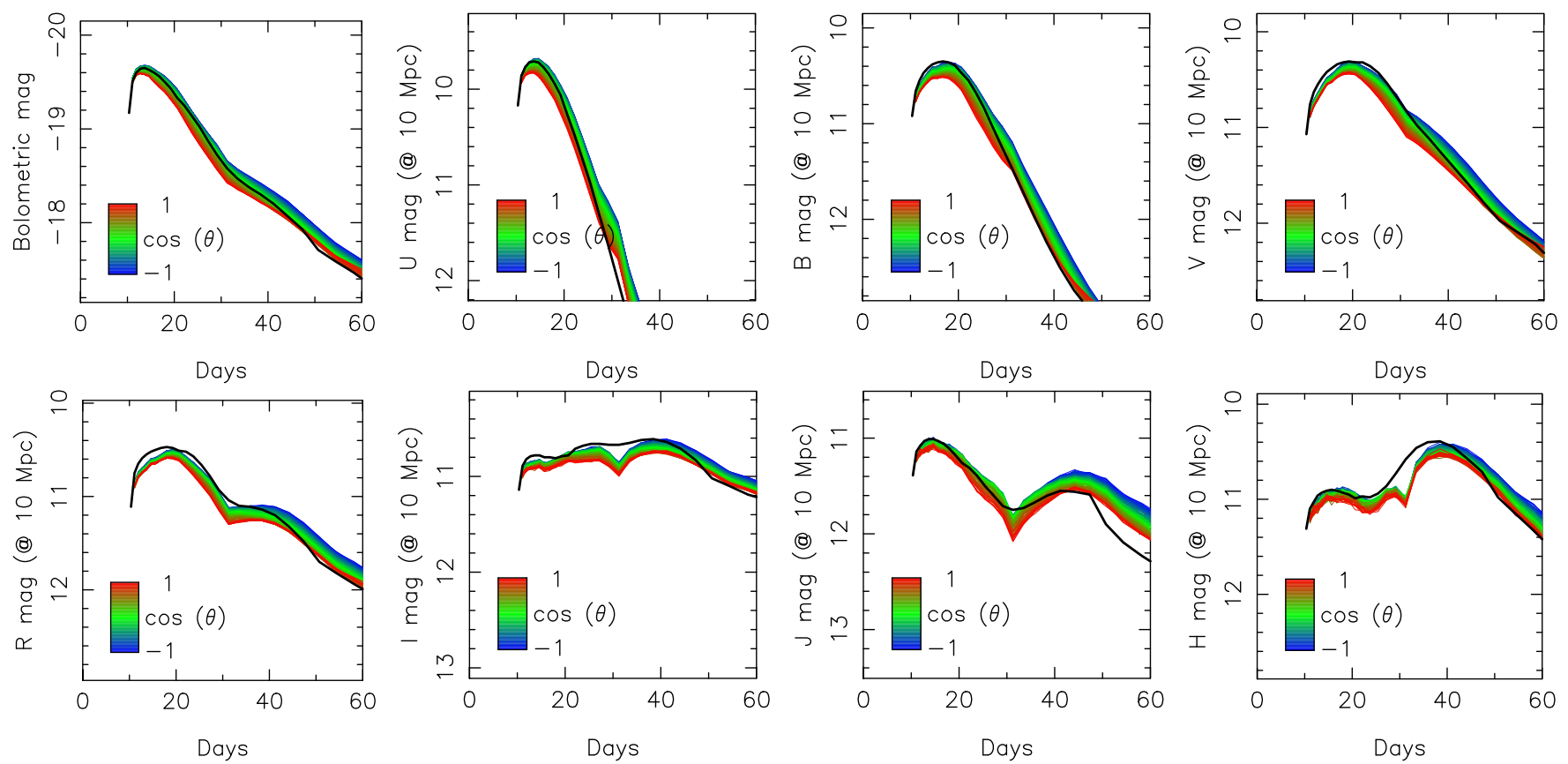

Figure 2. Simulated multiband light curves for Model RGa. The color coordinates indicate the light curves from different viewing directions (red for $\theta=0$ and blue for $\theta=\pi$ ). The reference model curve is shown by the solid black curve. The variation due to different viewing directions is modest, at the 0.1 mag level in all of the bands. Also, it is fainter if viewed from the companion direction $(\theta=0)$.

(A color version of this figure is available in the online journal.)

a standard set of opacities largely used in radiation transfer simulations in the expanding SN ejecta: electron scattering, free-free, bound-free, and bound-bound transitions. In this paper, we adopt the expansion opacity prescription and two-level approximation for the discrete transitions. This introduces one parameter in dealing with the discrete transitions, called the thermalization parameter $\epsilon$. We adopt $\epsilon=0.3$ in our simulations (see Appendix A).

Throughout the simulation, frequency, energy, and directions of photon packets are recorded for those escaping the simulation region (i.e., SN ejecta) within the simulated time interval, together with the time of the escape. This information is summed up at the end of the simulations to reconstruct the escaping radiative flux from the system. In this process, the photons are binned into 100 polar directions from 0 to 180 degrees spaced equally in the solid angle and into wavelength bins that are discretized into 3000 bins logarithmically spaced in the frequency space from $100 \AA$ to $20000 \AA$. The time bins are divided into 30 from 10 days to 80 days, equally spaced in a logarithmic scale. In practice, we performed the first MC transfer simulations with a smaller number of photon packets $\left(\sim 10^{8}\right.$ in total) to converge thermal conditions in the ejecta. Then adopting this thermal structure, we performed the final MC transfer with a larger number of photon packets $\left(\sim 10^{9}\right)$ to obtain a sufficiently high signal-to-noise $(\mathrm{S} / \mathrm{N})$ ratio in the resulting angle- and time-dependent spectra. This large number of photon packets allows us to extract smooth spectra with high $\mathrm{S} / \mathrm{N}$; namely, on average the number of photons in each timewavelength-angle bin is $\gtrsim 100$.

\section{LIGHT CURVES AND SPECTRA}

Here we present the results of our simulations. We frequently comment on the viewing angle to an observer. In the following sections, we denote the viewing angle by $\theta$. The viewing angle $\theta$ is defined to be a polar angle as measured from the direction toward the companion star (or the hole). Namely, $\theta=0$ for an observer viewing the $\mathrm{SN}$ from the companion direction, and $\theta=\pi$ for one viewing it from the opposite direction.

Figures 2-4 show synthetic multiband light curves of Models $\mathrm{RGa}, \mathrm{RGb}, \mathrm{MS}$, respectively. Those for our reference noninteraction model are also shown for comparison. Despite the large asymmetry in the ejecta structure (Figure 1), the light curves of the interaction models are found to be very similar to those without interaction. The difference between different companion types is even smaller, and there is virtually no difference visible down to the resolution in our simulations. We note that the deviation of the noninteraction model light curve in the $J$ band from the interaction models is presumably a numerical artifact, as is a spiky feature in the light curves of Model RGa around 30 days. We note that this spiky feature (apparently a nonconverged temperature at this epoch) would not affect the later evolution because we solve the temperature convergence at every time step.

A close inspection shows that around the peak the noninteraction model is almost identical to the interaction model viewed at $\theta \sim \pi$ (opposite to a companion or a hole). In the later phase, the noninteraction model is fainter than the angle-averaged mean light curves from the interaction model. This is consistent with the expectation: early on, the noninteracting side should look like the noninteraction model because one would not see the other side. Later on as the photon diffuses out, one will eventually see effects of the interaction, and the bolometric luminosity should eventually follow the $\gamma$-ray deposition rate as the ejecta become transparent to optical photons. At this moment, the bolometric luminosity is approximated by a simple gamma-ray deposition rate (see, e.g., Maeda et al. 2003), which is given as $L \propto M_{\mathrm{ej}}^{2} / E_{K}$ (where $L$ is the bolometric luminosity and $M_{\mathrm{ej}}$ and $E_{K}$ are the ejecta mass and the kinetic energy, respectively). Here, while $E_{K}$ should be unaffected by the interaction as the total energy is conserved, the ejecta mass is larger for the interaction case than for the noninteraction case because of the 

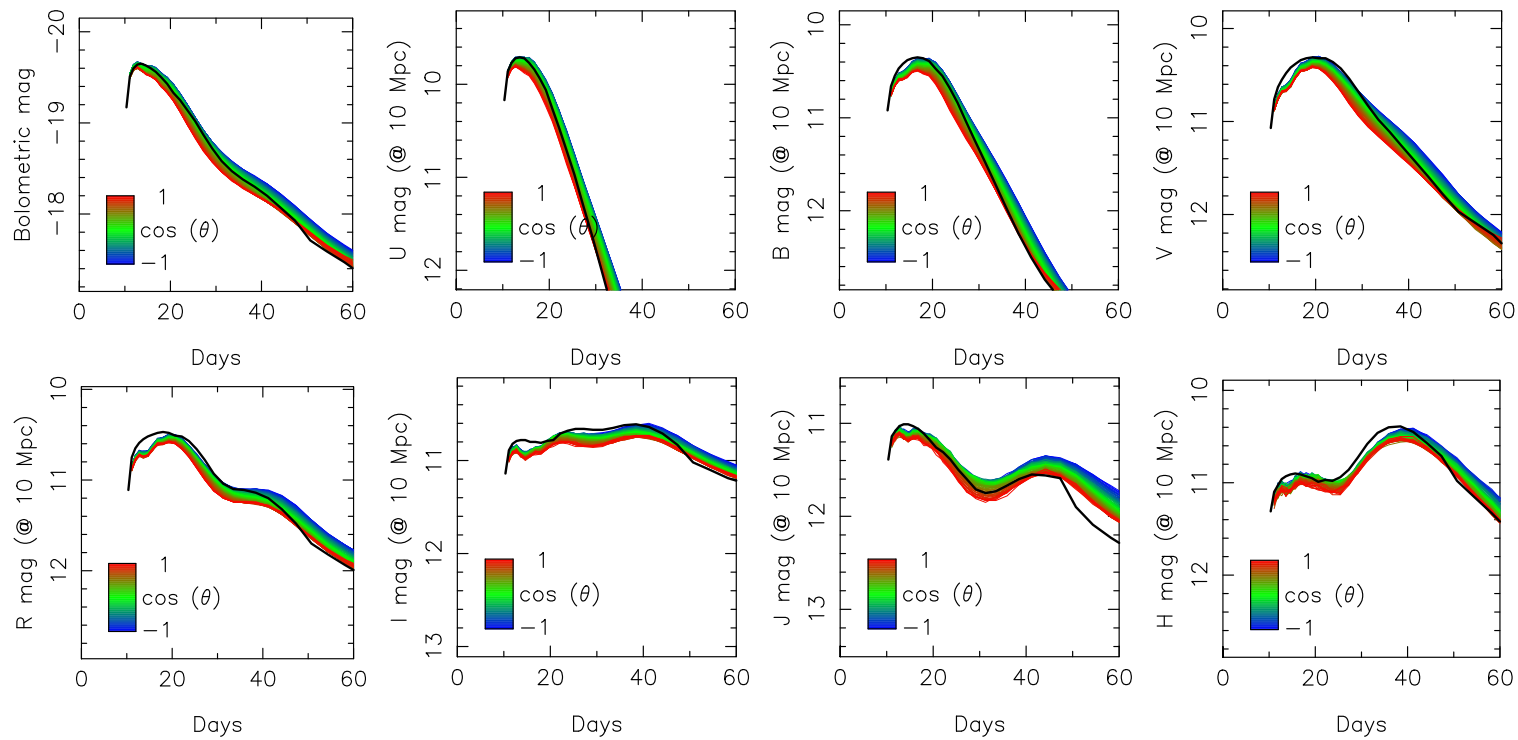

Figure 3. Simulated multiband light curves for Model RGb.

(A color version of this figure is available in the online journal.)
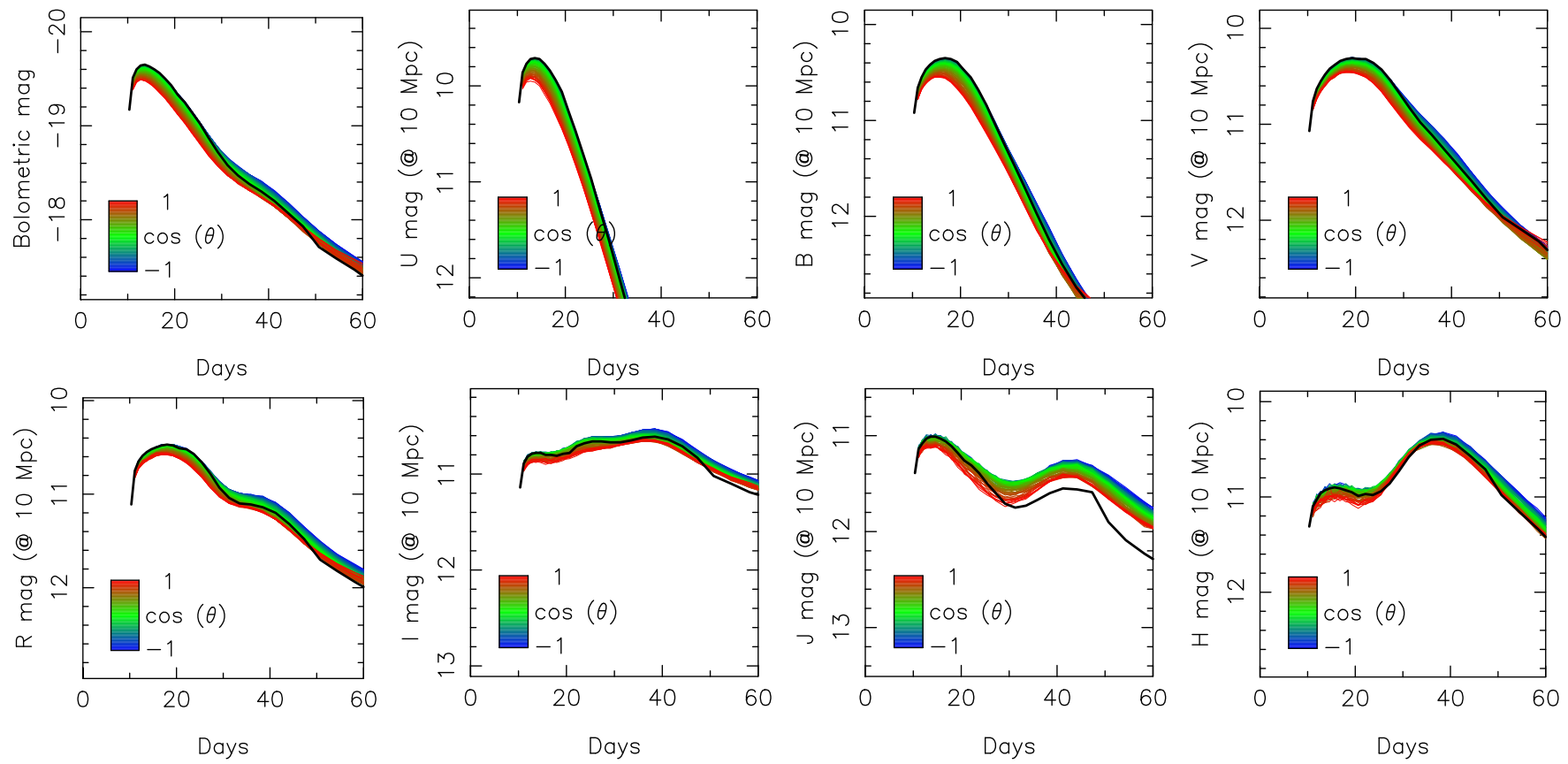

Figure 4. Simulated multiband light curves for Model MS.

(A color version of this figure is available in the online journal.)

addition of the envelope mass stripped from the companion. Namely, there is a larger amount of material in the interaction case to absorb more $\gamma$-rays than the original ejecta without interaction. Naively, one would expect that the $\gamma$-ray deposition efficiency would be larger in the interaction model $\left(M_{\mathrm{ej}} \sim 1.8 M_{\odot}\right)$ than in the noninteracting model $\left(\sim 1.4 M_{\odot}\right)$ by $\sim 60 \%$ if the deposition rate is simply scaled by the ejecta mass. This would lead to a late-time bolometric luminosity of the interaction model larger than the original by $\sim 0.5 \mathrm{mag}$. This is enough to explain the difference in the late-time luminosity at the $\sim 0.1-0.2 \mathrm{mag}$ level in the two models. Note that a difference as large as $0.5 \mathrm{mag}$ is the maximum difference we expect because the asymmetry in the ejecta in the interaction model is expected to lead to more effectively escaping $\gamma$-rays than in a spherical model with the same ejecta mass.
The variation arising from different viewing directions is at the level of $0.1 \mathrm{mag}$, and an SN looks generally fainter for directions closer to the companion/hole $(\theta \sim 0)$ in all of the bands. Because we have found little difference for different models, in the rest of the paper we mainly focus on Model RGa.

Figures 5 and 6 show the synthetic spectral time sequence in the optical wavelengths and in the NIR wavelengths, respectively. As expected from the light curves, the interaction with the companion is also found not to create any dramatic effects in the spectra. Namely, existence of a close binary nondegenerate companion does not leave detectable features in overall spectra around the maximum light and thereafter (i.e., 10-80 days after the explosion), and, in other words, this does not conflict with the observed uniformity of SN Ia spectra in these epochs. 

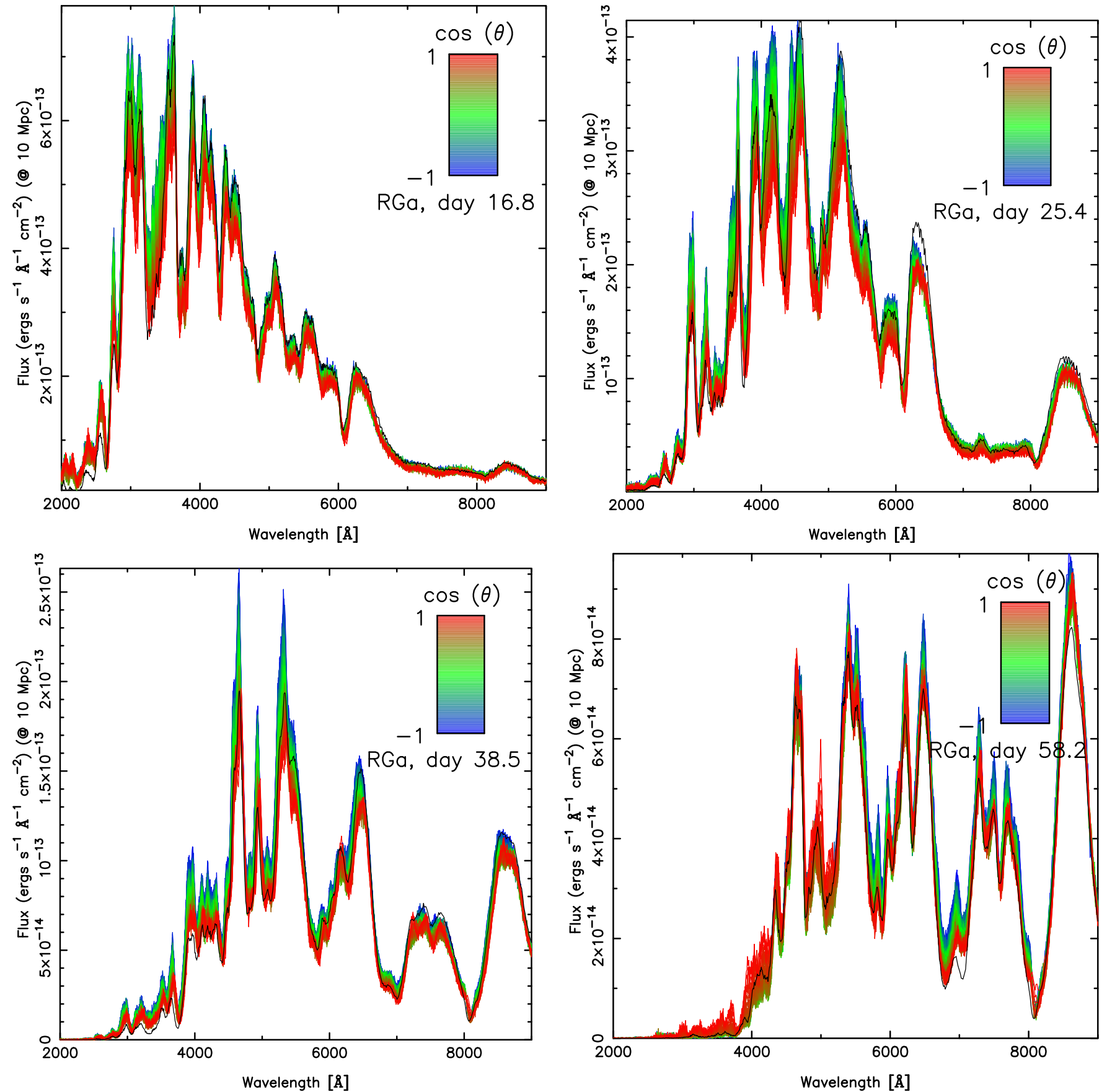

Figure 5. Simulated spectra in optical wavelengths for Model RGa. The color coordinates indicate the spectra from different viewing directions (red for $\theta=0$ and blue for $\theta=\pi$ ). When viewed from the companion (hole) direction $(\theta=0)$, the spectra are redder, with the smaller flux especially in the blue, than viewed by the opposite-side observer $(\theta=\pi)$. The difference is at a moderate level, so the spectra would not be classified as peculiar.

(A color version of this figure is available in the online journal.)

Still, there is a difference. When viewed from the companion (hole) direction $(\theta=0)$, the spectra are redder, with the smaller flux especially in the blue, than as viewed by the opposite-side observer $(\theta=\pi)$. The difference is at a moderate level, so the spectra would not be classified as peculiar, and this SN would be classified into the same class irrespective of the viewing directions. Rather, this would create diversity, especially in the intrinsic color, within the same classification.

The temperature and ionization structures are shown in Figures 7 and 8, respectively. Overall, the temperature is lower on the side of the companion due to a smaller amount of the heating source $\left({ }^{56} \mathrm{Ni}\right)$ and also due to a smaller amount of absorbing matter (heavy elements, especially Fe-peak elements) on this side than the others.

We note that our results are qualitatively different from those found by Kasen et al. (2004), who predicted that the SN is bluer and brighter (especially in the shorter wavelengths) when viewed at $\theta \sim 0$. The situation that an observer at $\theta \sim 0$ views directly in the ${ }^{56} \mathrm{Ni}$-rich region was a main cause of the predicted behavior by Kasen et al. (2004). Kasen et al. (2004) performed a snapshot spectral synthesis for a maximum spectrum based on a toy model. In their model, they mimicked the outcome of the interaction as the SN ejecta with a hole represented by a (nearly) constant opening angle. We note that 

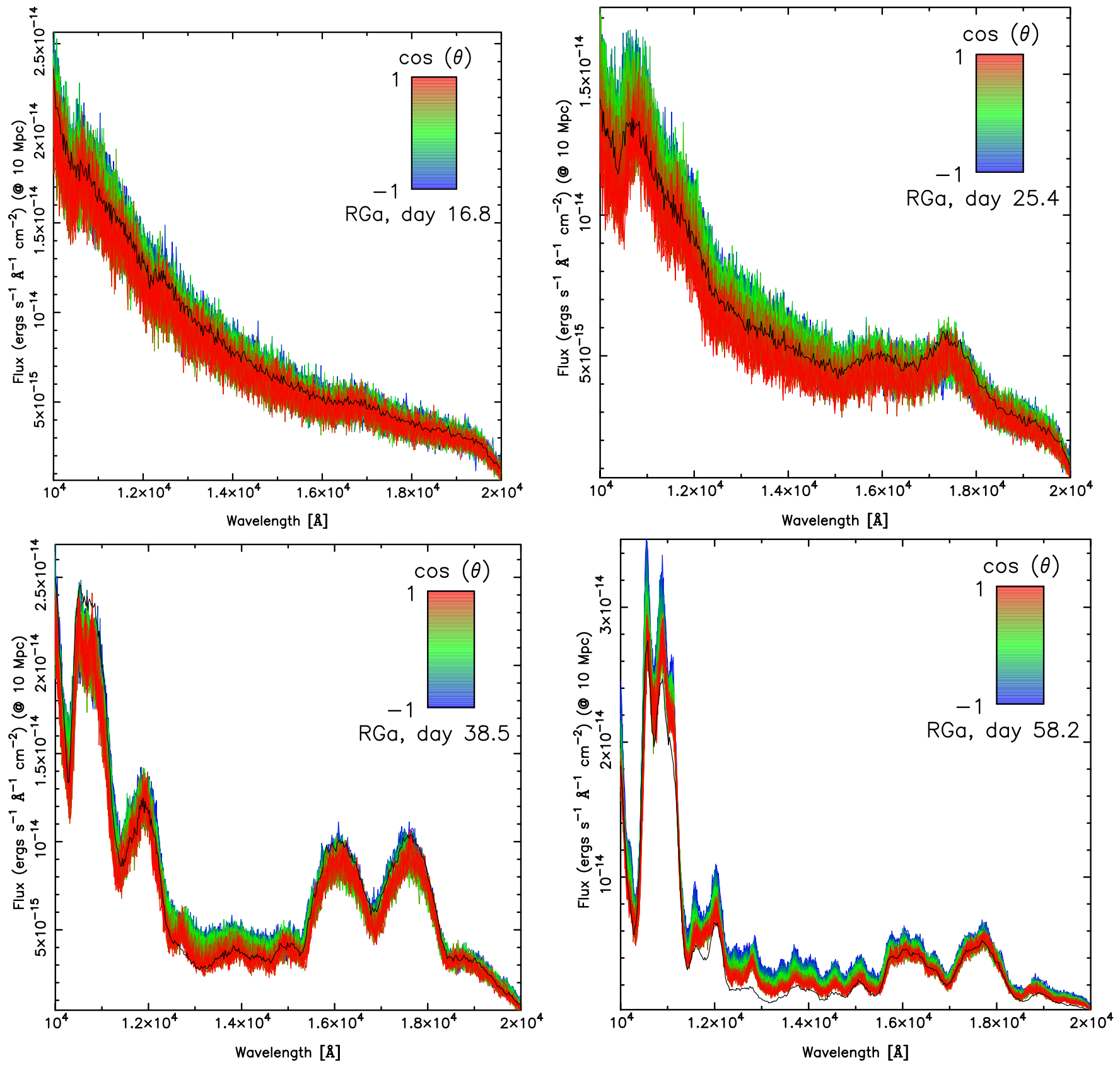

Figure 6. Simulated spectra in NIR wavelengths for Model RGa. (A color version of this figure is available in the online journal.)
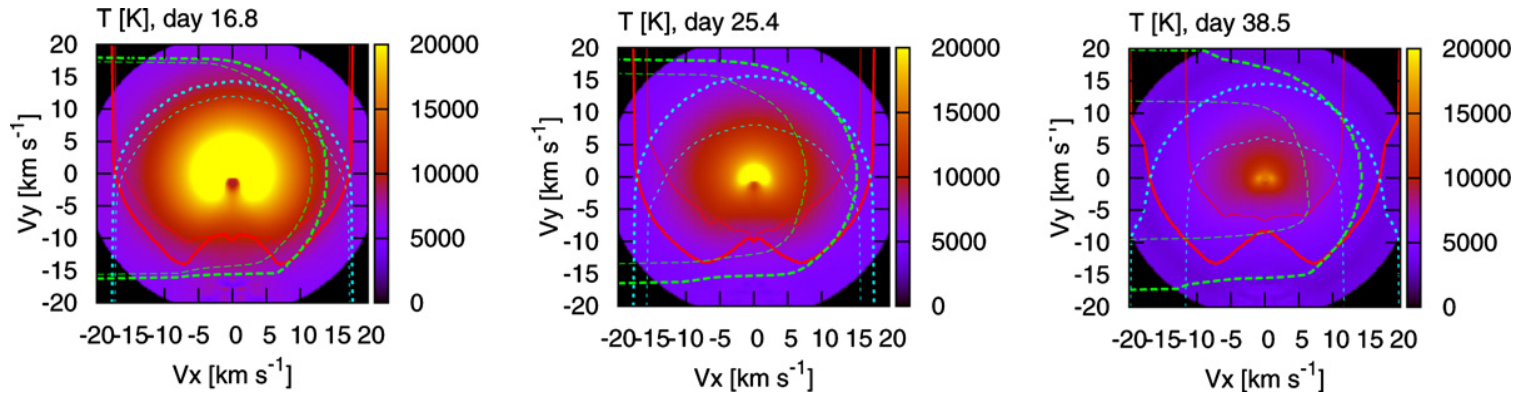

Figure 7. Temperature distribution of Model RGa. Also shown here are the $U$-band (thick) and $R$-band (thin) photosphere positions (as defined by $\tau=2 / 3$ ). The photosphere is shown for an observer at $\theta=0$ (red), $\theta=\pi / 2$ (green), and $\theta=\pi$ (blue).

(A color version of this figure is available in the online journal.) 

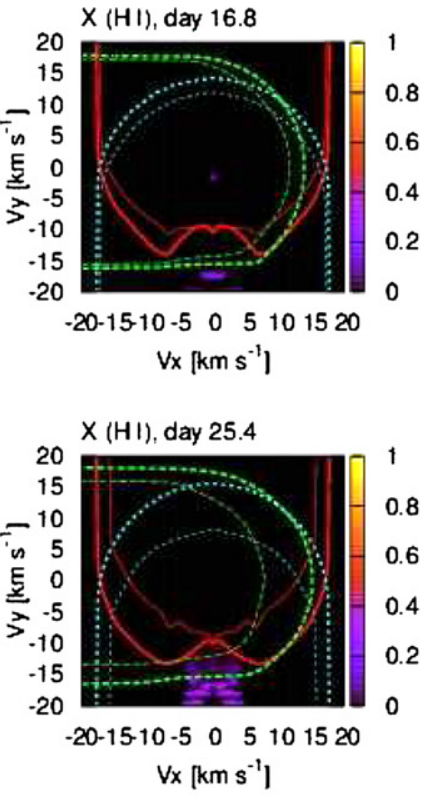

$\mathrm{X}(\mathrm{HI})$, day 38.5

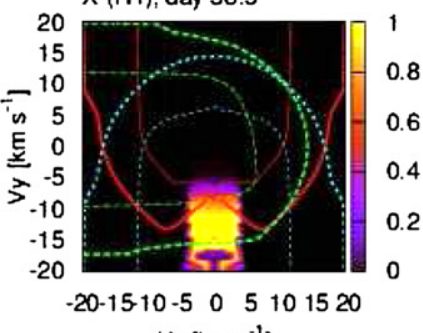

$\mathrm{Vx}\left[\mathrm{km} \mathrm{s}^{-1}\right]$
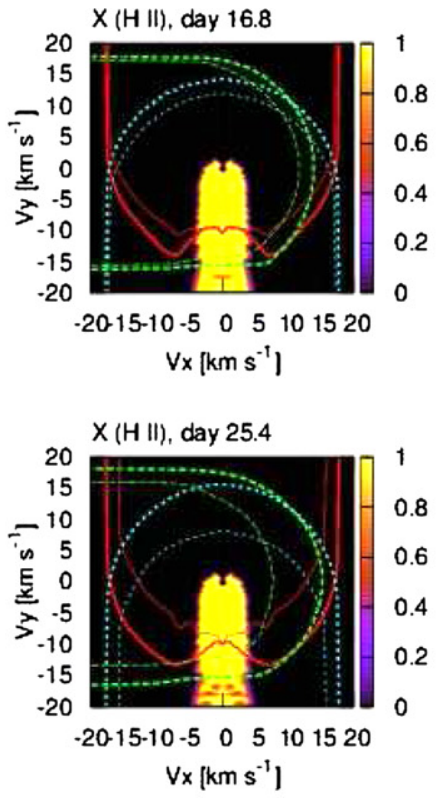

$X(\mathrm{H}$ II), day 38.5

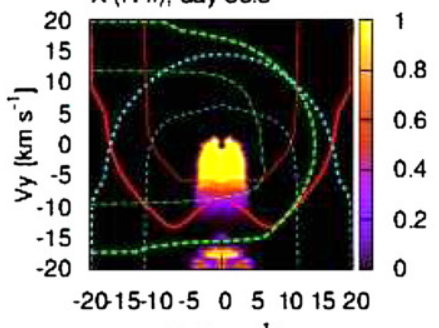

$\mathrm{V} \times\left[\mathrm{km} \mathrm{s}^{-1}\right]$
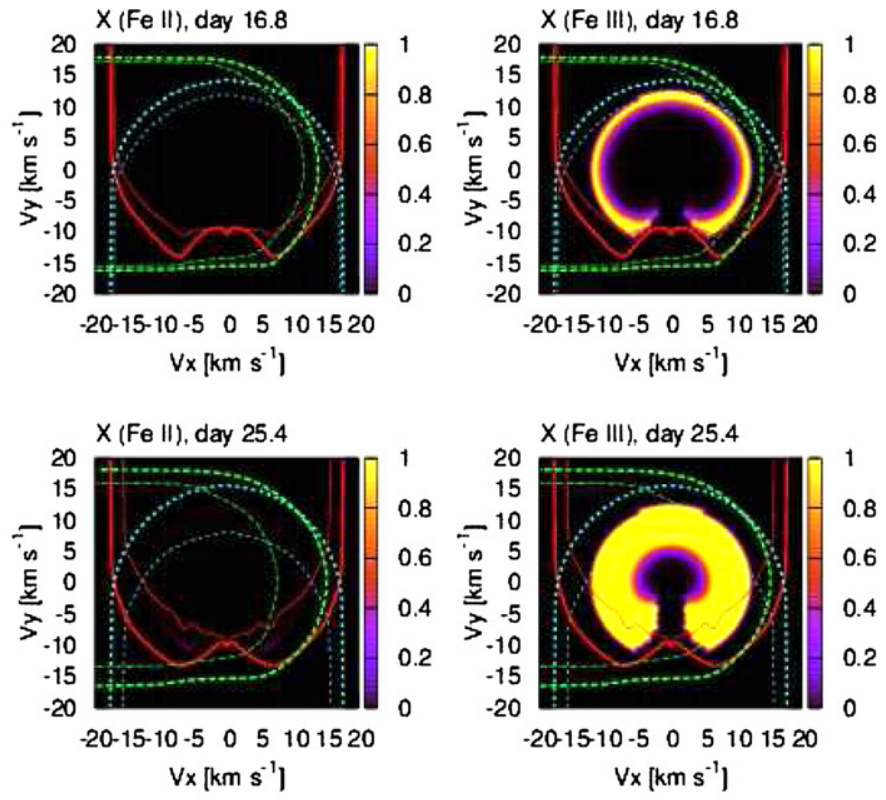

$X(\mathrm{Fe} I 1)$, day 38.5

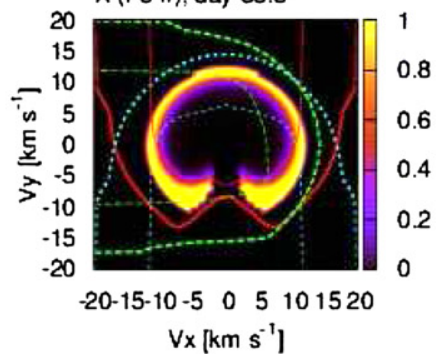

$X(\mathrm{Fe}$ III), day 16.8

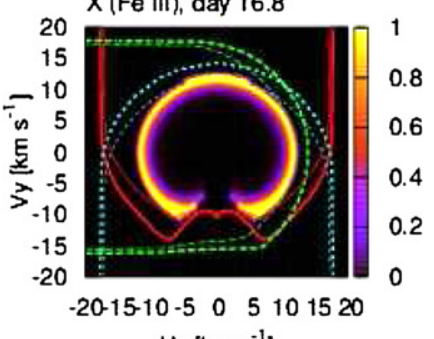

$\mathrm{Vx}\left[\mathrm{km} \mathrm{s}^{-1}\right]$

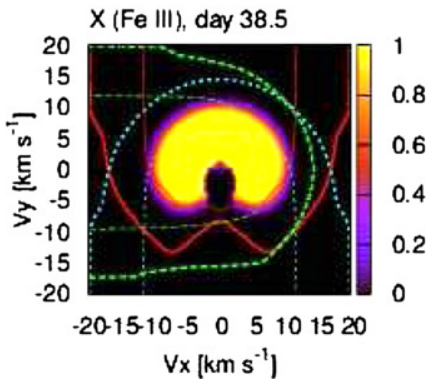

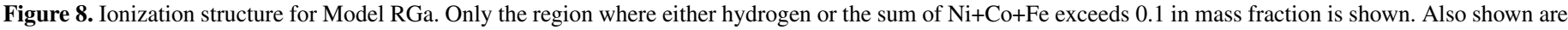
the positions of the $U$-band (thick) and $R$-band photospheres (see the caption of Figure 7).

(A color version of this figure is available in the online journal.)

their model underestimates the amount of material in the hole: a large amount of the $\mathrm{SN}$ ejecta, either a $\mathrm{C}+\mathrm{O}$ or $\mathrm{Si}$-rich layer, fills up the hole left by the interaction, and also the H-rich companion materials are naturally filling the hole as well. Thus the hole is not really a vacuum. The existence of these materials does not allow the photosphere to quickly recede to the bottom of the hole (i.e., the ${ }^{56} \mathrm{Ni}$-rich central region). Even with only the H-rich envelope from the companion intruding into this region, electron scattering can become significant (especially for the RG case) to clip the photosphere at a relatively high velocity; with $\sim 0.5 M_{\odot}$ of fully ionized hydrogen materials (with 70\% mass fraction) confined within a sphere below $1000 \mathrm{~km} \mathrm{~s}^{-1}$, the electron scattering optical depth is estimated to be $\tau \sim 2700(t / 20 \text { days })^{-2}$. Thus, this will hide the ${ }^{56} \mathrm{Ni}$-rich region from the line of sight of an observer at $\theta \sim 0$ until the hydrogen recombines. The hydrogen is kept nearly fully ionized during the epochs of interest in this paper (Figure 8), especially in the low-velocity region, where $\gamma$-rays and optical photons are absorbed efficiently due to the large density.

In our situation, based on the hydrodynamic model, the temperature at the photosphere at $\theta \sim 0$ tends to be lower than that at $\theta \sim \pi$ because the companion direction $(\theta \sim 0)$ is blocked by the ${ }^{56} \mathrm{Ni}$-free materials. This direction lacks Fe-peak elements, and accordingly the photosphere at the $U$ band is at a low velocity. The position of the photospheres at the $R$ band is not extremely sensitive to the viewing direction, supporting the interpretation that the main difference in the photosphere in the $U$ band is caused by the different amount of Fe-peak elements that are sources of opacity, especially in blue bands. The $U$-band photosphere does not quickly recede in the velocity space as compared to the $R$ band, for observers viewing from any direction, which reflects the increasing opacity in the blue at the later epochs due to recombination of Fe-peak elements (Figure 8).

Figure 9 shows the evolution of the spectral region around Si II 6355. Around the peak luminosity (i.e., $\sim 15$ days after the explosion), the absorption minimum (and the emission peak) is at a longer wavelength (i.e., lower velocity) for observers at smaller $\theta$, as is consistent with the result by Kasen et al. (2004). We find that the predicted temporal evolution is also different for different viewing directions (see also Kutsuna \& Shigeyama 2013; Kutsuna 2013). Indeed, at $\sim 10$ days after the explosion (i.e., about a week before the $B$-band maximum), the line profiles are not sensitively dependent on the viewing direction. After that, the line velocity decreases more quickly for an observer at $\theta \sim 0$, thus leading to progressively lower velocity for this direction. This temporal behavior is also different from that predicted by Kutsuna \& Shigeyama (2013), who predicted the lower velocity of the Si II for $\theta \sim 0$ than for $\theta \sim \pi$ already well before the maximum. Figure 10 demonstrates how the line profiles are different for different viewing directions at 16.8 and 38.5 days after the explosion. At 38.5 days, it is not easy to identify the $\mathrm{Si}$ II in the spectra. We note that the line profile at $\sim 6150-6200 \AA$ is different for different viewing directions, showing a small peak for $\theta \sim 0$ but not for $\theta \sim \pi$. This wavelength is influenced by the material moving at the velocity 

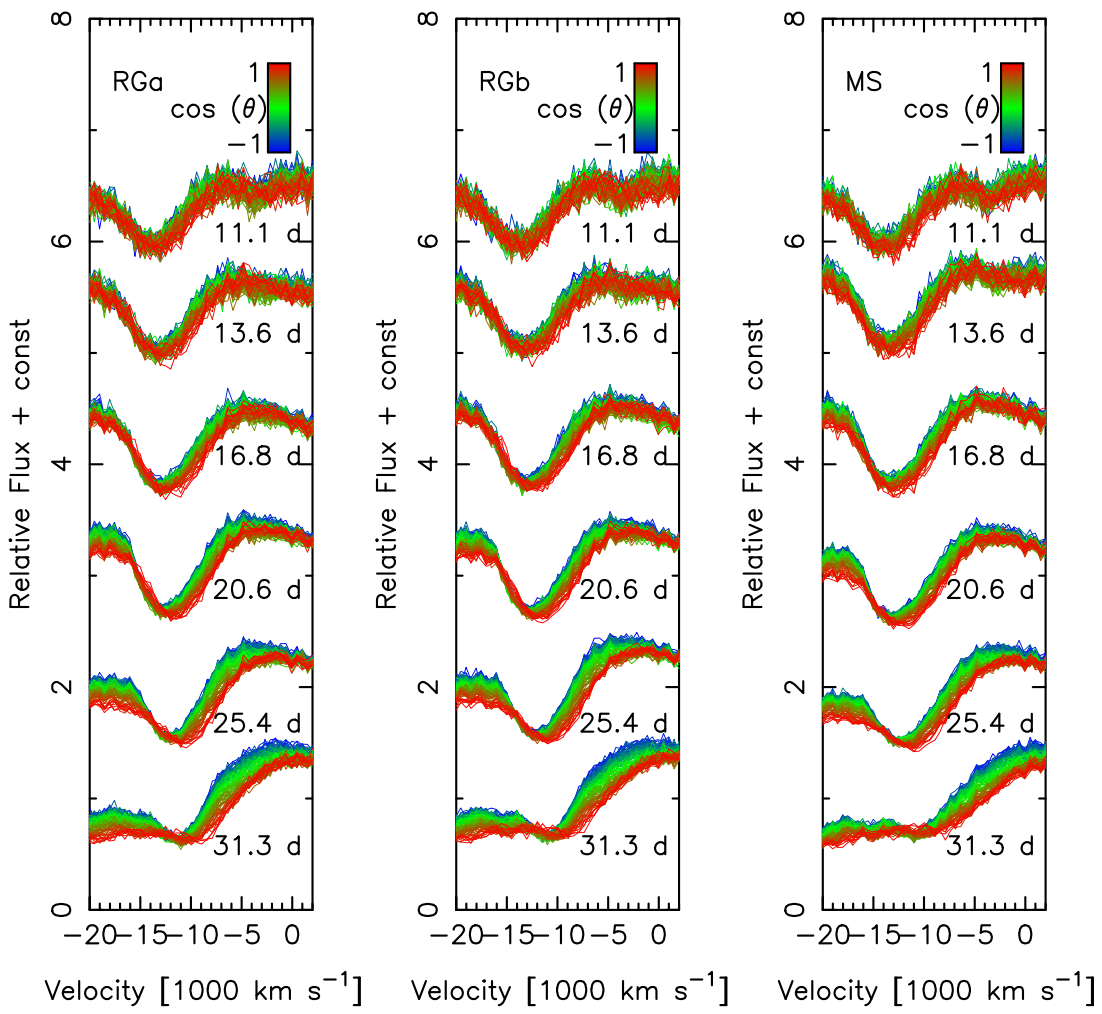

Figure 9. Si II 6355 in the simulated spectra. The color coordinates indicate the spectra as viewed from different viewing directions (red for $\theta=0$ and blue for $\theta=\pi$ ). Initially at $\sim 10$ days the Si II profile is similar for observers at any direction, and then observers at smaller $\theta$ (toward the companion direction) will observe the Si II at progressively lower velocity than in the opposite direction.

(A color version of this figure is available in the online journal.)
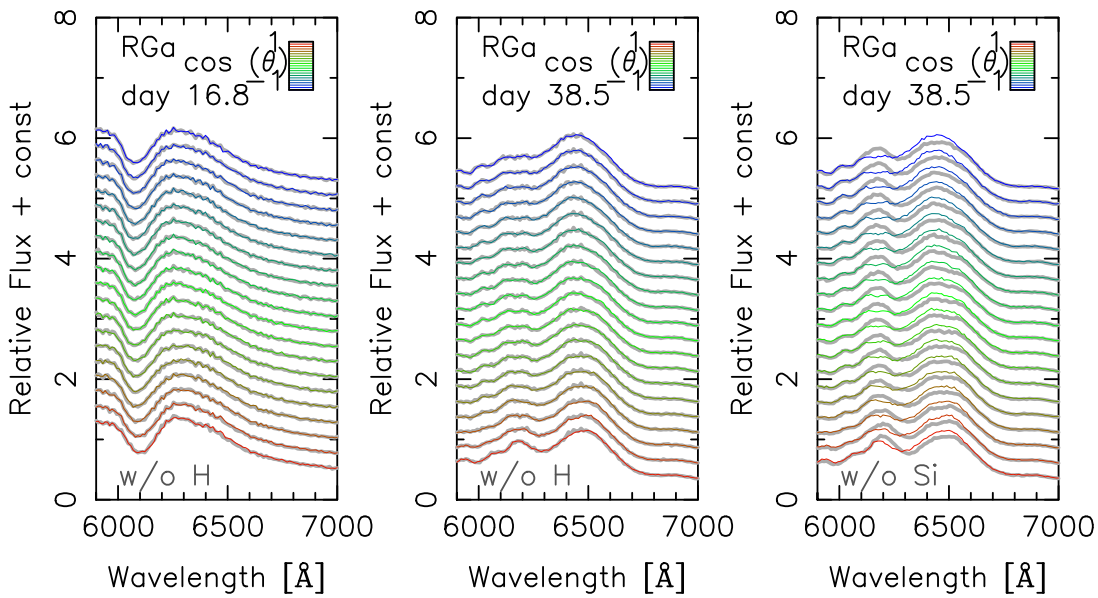

Figure 10. Simulated spectra around Si II 6355. The color coordinates indicate the prediction for different viewing directions (red for $\theta=0$ and blue for $\theta=\pi$ ). In this figure, the spectra for observers at different directions are added with an additional offset $(\theta=0$ to $\pi$, from bottom to top). In the left two panels, the color curves are the model curves with hydrogen, and the gray curves are without hydrogen. The color curves shown in the right panel are identical to the one in the middle (i.e., Model RGa at day 38.5 after the explosion), and the gray curves are without silicon. $\mathrm{H}_{\alpha}$ is observationally not detectable in both epochs. The viewing angle dependence is small around the $B$-band maximum, and the variation arising from Si II 6355 becomes visible in the later epoch.

(A color version of this figure is available in the online journal.)

of $\sim 7000-10,000 \mathrm{~km} \mathrm{~s}^{-1}$. Figure 10 (right panel) shows that $\mathrm{Si}$ iI 6355 affects this wavelength range differently for different viewing directions.

Figure 11 shows the Sobolev optical depth of Si II together with the $R$-band photosphere. Around the peak luminosity, the peak in the opacity distribution is on average at a lower line-of-sight velocity for $\theta=0$ because the highest velocity materials are missing in this direction. At the epoch of the peak luminosity (at day 16.8), this particular optical depth is larger at $\theta=0$ because the lower temperature there prefers $\mathrm{Si}$ II over Si III. As time goes by, the temperature decrease induces the recombination of $\mathrm{Si}$ II to $\mathrm{Si}$ I. The ejecta on the companion side $(\theta \sim 0)$ do not keep the high optical depth of Si II at day 38.5, whereas the ejecta on the opposite side $(\theta \sim \pi)$ still maintain a sufficient amount of $\mathrm{Si}$ II to keep the line velocity as high as $\sim 8000 \mathrm{~km} \mathrm{~s}^{-1}$. As a result, at this epoch the flux around $6200 \AA$ is suppressed for $\theta \sim \pi$ but not for $\theta \sim 0$. In principle, this small but varying feature in Si II 6355 could be a powerful 

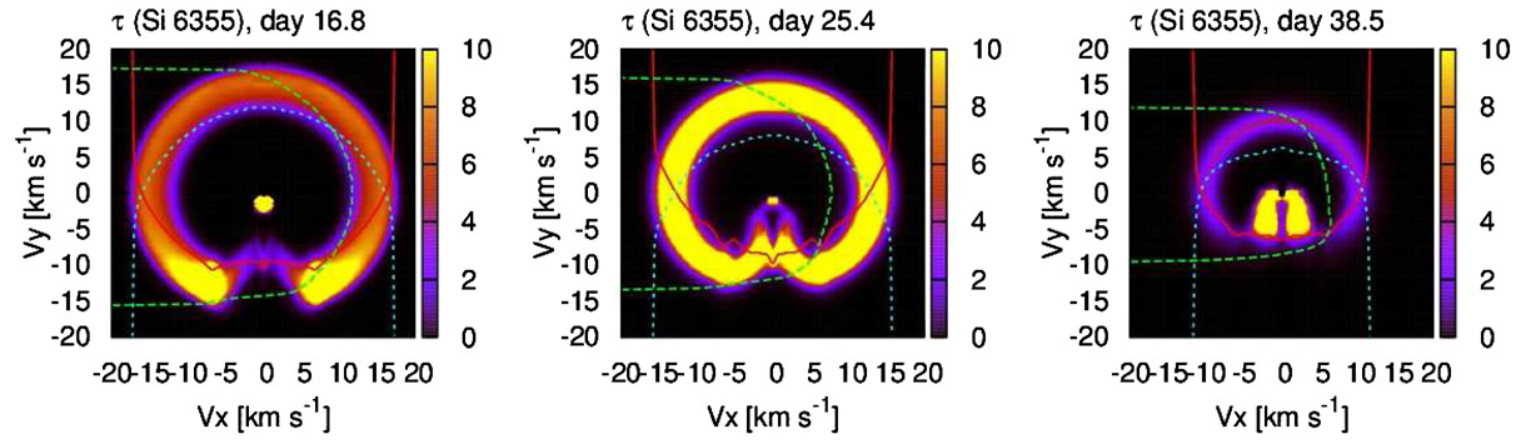

Figure 11. Sobolev optical depth of Si II 6355 for Model RGa. Shown here is the $R$-band photosphere $(\tau=2 / 3$ ) for an observer at $\theta=0$ (red), $\theta=\pi / 2$ (green), and $\theta=\pi$ (blue).

(A color version of this figure is available in the online journal.)
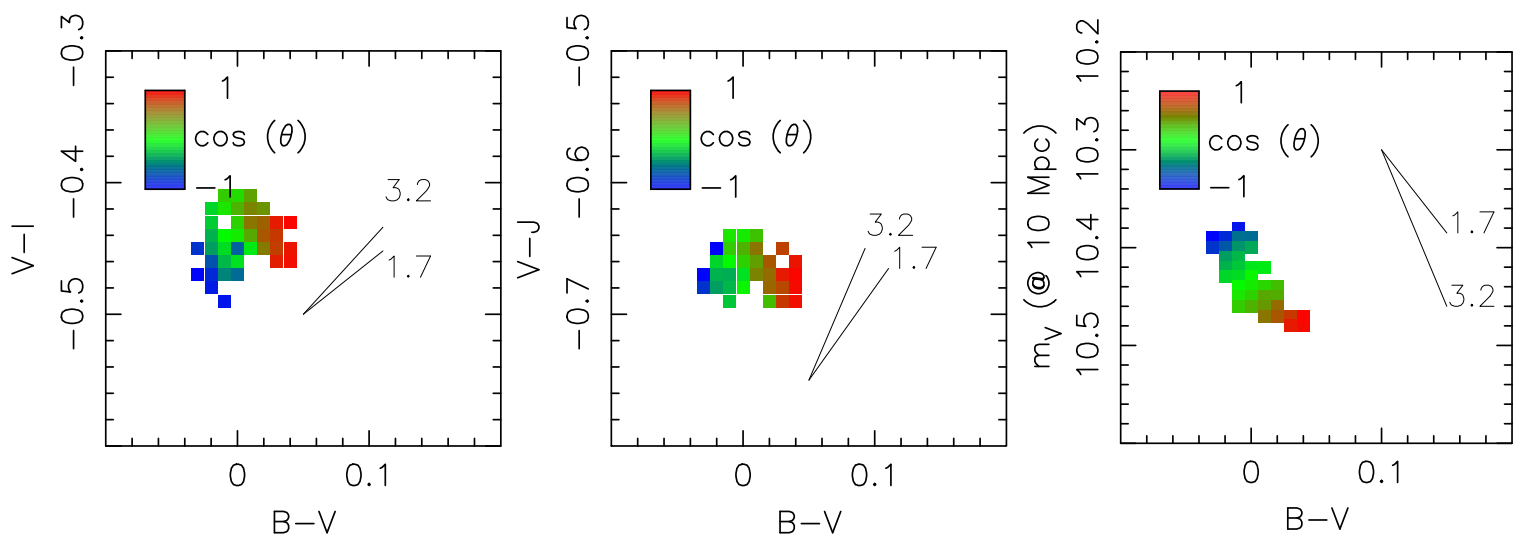

Figure 12. Predicted relations in photometric properties for Model RGa. The color coordinates indicate the prediction for different viewing directions (red for $\theta=0$ and blue for $\theta=\pi$ ). The external-extinction vector is shown for $R_{V}=3.2$ and 1.7. The variation in each quantity is at most at the level of $0.1 \mathrm{mag}$. The trend seen in the optical properties is similar to the effect of external extinction, but it is not the case in the NIR.

(A color version of this figure is available in the online journal.)

signature of the presence of a companion star, and this is further discussed in Section 6.

\section{SPECTRAL FEATURES AND COLOR}

In Section 3, we showed that the overall light curve and spectral behaviors are not much affected by the existence of a nondegenerate companion star. Still, we find a small variation for different viewing directions, and in this section we investigate more details on this issue.

Figure 12 shows the variations in the colors and the $V$-band magnitude and their relations to the $B-V$ color obtained for Model RGa. The variation in each quantity is at the level of $0.1 \mathrm{mag}$ or even smaller, so such an effect is difficult to see in current observations, and it is practically impossible to disentangle this effect from other possible sources of the scatter. Relations between different observables, however, have interesting implications. Within the optical range, the relations between different colors are indeed similar to the effect of external extinction. This is also true for relations between color and absolute magnitude. This means that the viewing angle variation can mimic the external extinction and could be a part of the origins of the scatter in the luminosity calibration (see, e.g., Maeda et al. 2011). This degeneracy could be solved by adding the NIR information because the optical-NIR colors are found not to follow the extinction vector.

The possible effect in the extinction estimate is demonstrated in Figure 13. Here, we show how the intrinsic color dispersion

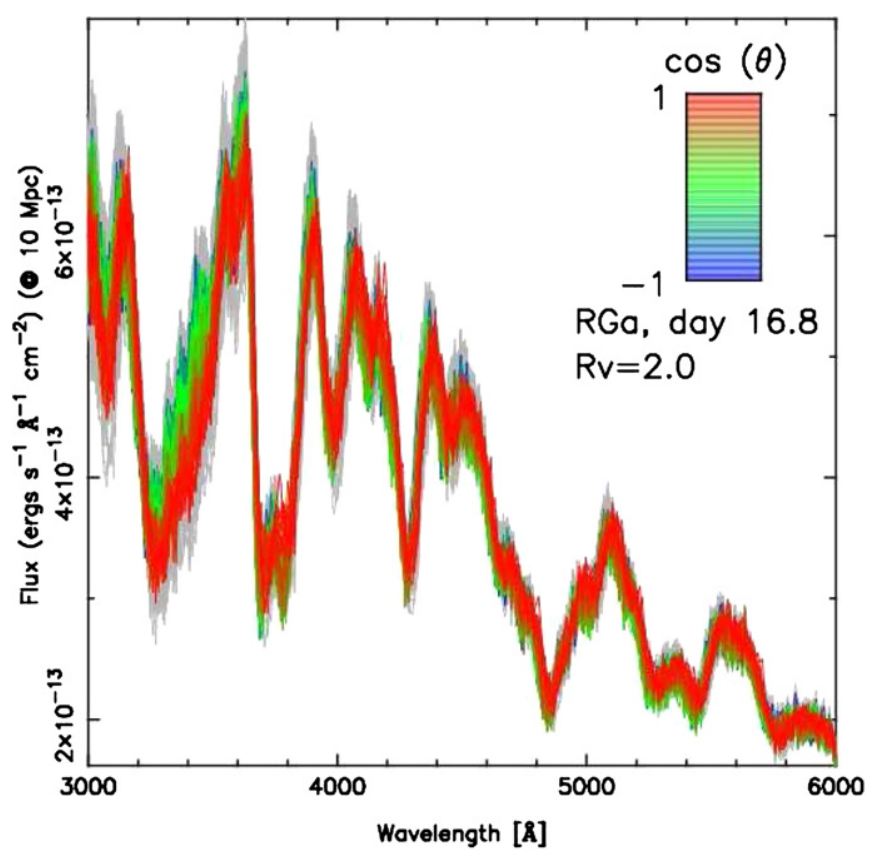

Figure 13. Demonstration of how the intrinsic color difference of Model RGa as arising from different viewing directions can be mimicked by the external extinction. The original spectra for various viewing directions are shown in gray, and the ones corrected for the hypothesized extinction are shown in colors.

(A color version of this figure is available in the online journal.) 

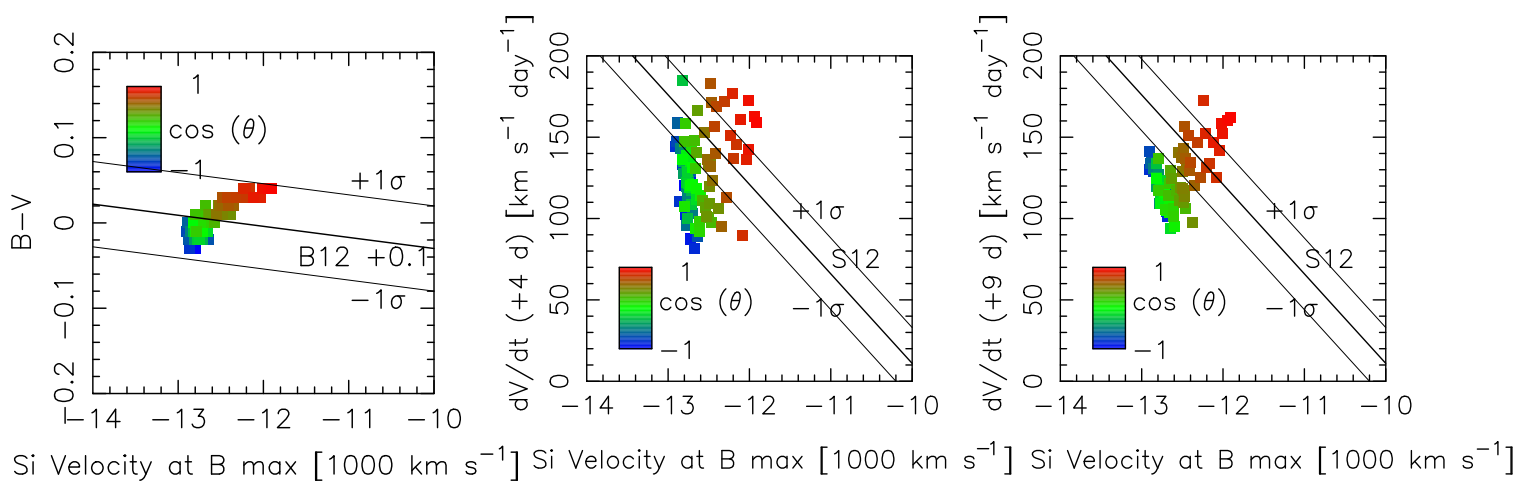

Figure 14. Predicted relations in spectroscopic properties for Model RGa. The color coordinates indicate the prediction for different viewing directions (red for $\theta=0$ and blue for $\theta=\pi$ ). The solid lines are for the observationally derived relations with the $1 \sigma$ statistical errors for the velocity-color (with an additional offset; Blondin et al. 2012, hereafter B12) and for the velocity-velocity gradient (Silverman et al. 2012, hereafter S12). The velocity gradient in the model is defined as the gradient in two epochs, where the first epoch is set at the $B$-band maximum and the second epoch is set either at four days or nine days after the $B$-band maximum. The relations due to the viewing angle diversity do not follow the observed relations. The expected diversities are within the observed scatters at the $1-2 \sigma$ level.

(A color version of this figure is available in the online journal.)

predicted for Model RGa can be mimicked by the external extinction. We hypothesized here (even though we know it is not the case) that the color variation here is entirely due to a different amount of the extinction, and we convolve the external extinction associated with the model $B-V$ color assuming $R_{V}=2.0$, as is typically derived for SNe Ia. Figure 13 shows that by doing it this way the dispersion in the optical spectra is reduced: if one observes Model RGa from various directions, one would associate the diversity in color to the external extinction. This would introduce a systematic error in the derived absolute magnitude at the $0.1 \mathrm{mag}$ level.

One of the biggest effects of the interaction is on the predicted velocity of absorption lines (Section 3). Indeed, the predicted relation between the velocity at the maximum light and the $B-V$ color and the one between the velocity and the velocity gradients are different from the observationally found relation. It has been reported that the absorption velocity around the maximum is correlated with $B-V$ in a way that the higher velocity SNe have redder intrinsic color (Foley \& Kasen 2011; Maeda et al. 2011; Blondin et al. 2012). Figure 14 shows the predicted relation between the Si II velocity and $B-V$ color, overlapped with the observed trend from Blondin et al. (2012) but with an added offset of $0.1 \mathrm{mag}$. Note that observationally the zero point in the intrinsic color is dependent on the determination of the reddening and also on the sample because the color is also dependent on $\Delta m_{15}$, and theoretically the absolute scale can be more sensitive to numerical details than the relative values (see Appendix B). Here we are interested in the trend and diversity. Figure 14 shows that the predicted trend is different from the observed trend, while the variation is still within the $1 \sigma$ scatter of the observationally derived relation.

There is also an observationally found relation between the velocity and the velocity gradient. The velocity gradient is a measure of how quickly the absorption velocity decreases as a function of time (Benetti et al. 2004, 2005). The velocity gradient is larger (i.e., the velocity decreases more quickly) for $\mathrm{SNe}$ with larger velocity at maximum (e.g., Silverman et al. 2012). Figure 14 shows the relation predicted for Model RGa as compared to the observationally derived relation from Silverman et al. (2012). Observationally, the velocity gradient is defined by a linear or higher order fit to the velocity evolution just after the $B$-band maximum (Benetti et al. 2004, 2005; Blondin et al. 2012). The absolute values of the velocity gradient for specific objects are dependent on the time interval for the fit as well as the fitting function, while the overall tendency to larger gradients for higher velocity $\mathrm{SNe}$ is not sensitive to these choices (e.g., Blondin et al. 2012). Here, the velocity gradient in the model is defined as the gradient in two epochs for the sake of simplicity, where the first epoch is set at the $B$-band maximum. To check the robustness of the result, we vary the second epoch, and it is set either at four days or nine days after the $B$-band maximum in Figure 14. Although we are forced to adopt the second epoch, which is not very late (because the model does not reproduce the observed Si II profile around day 25 and thereafter; see Appendix A), we see that our results are not affected much by the definition of the velocity gradient (see Figure 14).

The velocity predicted for Model RGa is as high as those of the high-velocity (or high-velocity-gradient) $\mathrm{SNe}$, and the dispersion arising from different viewing angles alone does not explain the observed range of the $\mathrm{Si}$ II velocity (i.e., $10,000-12,000 \mathrm{~km} \mathrm{~s}^{-1}$ for the low-velocity SNe and reaching $\sim 16,000 \mathrm{~km} \mathrm{~s}^{-1}$ for the high-velocity $\mathrm{SNe}$ ). Specifically, the viewing angle effect arising from the asymmetry introduced by the ejecta-companion interaction is not a main cause of the diversity in the velocity (see, e.g., Maeda et al. 2010, for a possible origin of the diversity arising from the asymmetry in the explosion itself). Moreover, the viewing-angle effect here predicts a trend different from the observed one (Figure 14). As such, the observed relation can, in principle, be used as a constraint on the existence of a nondegenerate companion star. The dispersion predicted for Model RGa indeed exceeds the nominal $1 \sigma$ error in the observed relation. There are, however, quite a number of outliers in this relation (e.g., see Figure 6 of Silverman et al. 2012), so the present sample does not strongly reject the existence of an $\mathrm{RG}$ companion for a majority of SNe Ia. In any case, this relation is potentially a strong diagnostic in limiting the fraction of SNe Ia with a nondegenerate companion star.

In the above arguments, we have dealt with the small differences, due to various viewing directions, at the $0.1 \mathrm{mag}$ level. A question is whether our simulations are accurate enough down to this level. In Appendix B, we discuss this numerical accuracy issue in detail with test calculations, and we conclude that the results are not numerical artifacts.

\section{HYDROGEN LINES}

The hydrogen-rich matter stripped off from the companion star, being embedded in the SN ejecta, has been regarded as 

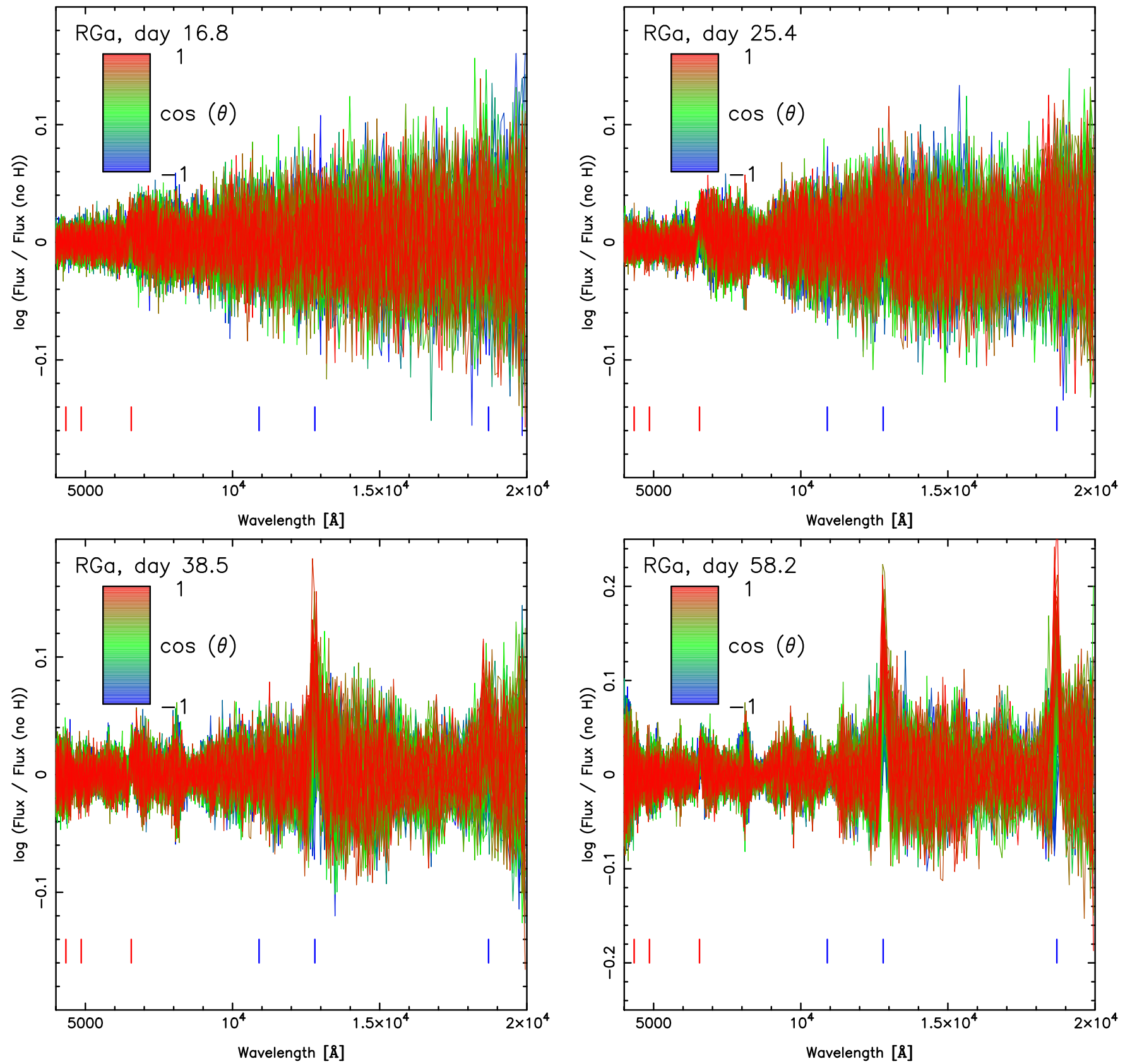

Figure 15. Ratio of the spectral flux with and without hydrogen lines for Model RGa. The color coordinates indicate the models for different viewing directions (red for $\theta=0$ and blue for $\theta=\pi$ ). The lines on the bottom show the rest wavelength positions of the Balmer series $(\alpha, \beta, \gamma)$ and the Paschen series $(\alpha, \beta, \gamma)$. In this plot, the simulated spectra are averaged in three time bins and three wavelength bins to increase the signal-to-noise ratio. In later phases, the variations in $\mathrm{P}_{\alpha}$ and $\mathrm{P}_{\beta}$ arising from different viewing directions clearly exceed the MC noise.

(A color version of this figure is available in the online journal.)

a key to probing (or rejecting) the existence of a nondegenerate companion star at the time of the SN Ia explosion. A large fraction of the stripped-off hydrogen is embedded near the center of the SN ejecta at low velocities and are only visible in the late phases when the SN ejecta become fully transparent. As such, a search for the $\mathrm{H}_{\alpha}$ emission in late-time spectra has been suggested and performed for a few SNe Ia (Mattila et al. 2005; Leonard 2007; Lundqvist et al. 2013; Shappee et al. 2013). A smaller amount of hydrogen is also distributed at high velocities (Marietta et al. 2000) and could be in principle probed by more easily accessible maximum and postmaximum spectra. However, the latter issue has not been quantitatively investigated in the past. Lentz et al. (2002) investigated whether the H-rich materials mixed into the high-velocity part of the SN ejecta ( through 1D radiation transfer simulations. They concluded that the signatures are expected to be stronger for the earlier phases, but the signals are generally weak. Recently, Kutsuna \& Shigeyama (2013) suggested that one may see $\mathrm{H}_{\alpha}$ even just after the maximum light (i.e., $\gtrsim 10$ days after the explosion) for the interaction with a nondegenerate companion, based on hydrodynamic simulation and simplified radiation transfer. Here, based on the same hydrodynamic simulation models with Kutsuna \& Shigeyama (2013) and detailed radiation transfer, 

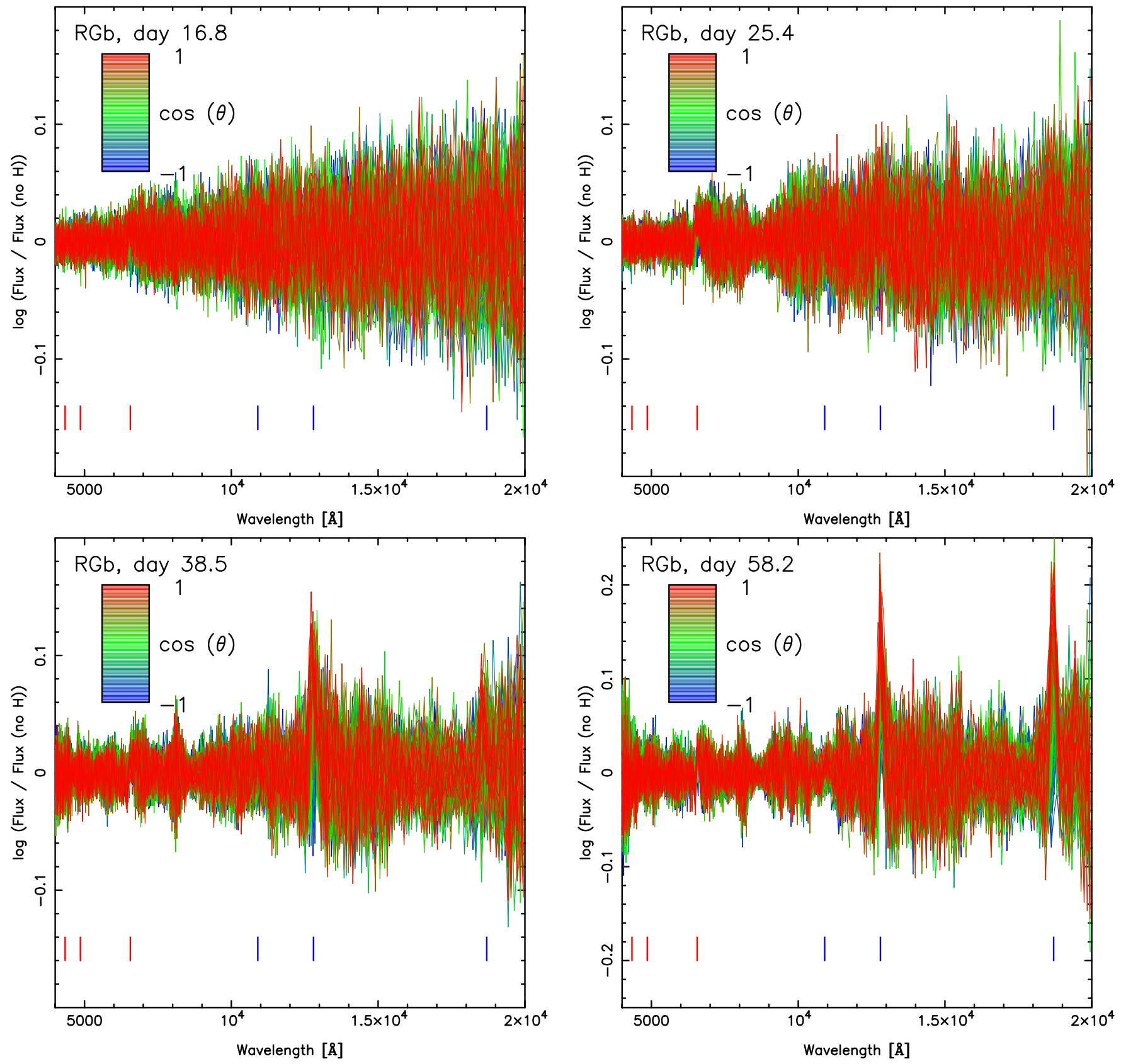

Figure 16. Ratio of the spectral flux with and without hydrogen lines for Model RGb. (A color version of this figure is available in the online journal.)

we investigate whether the existence of hydrogen is visible in the maximum and postmaximum spectra (i.e., up to about a month after the $B$-band maximum).

Figures 15-17 show the ratios of the synthetic spectra for the same model(s) but with and without hydrogen included in the radiation transfer. To create the hydrogen-free reference spectra, we performed the same radiation transfer simulation based on the temperature structure obtained through the original calculations, but setting the bound-bound and bound-free hydrogen opacities to zero by hand. In Figures 15-17, the model results for different directions are shown by different colors (red for $\theta=0$ and blue for $\theta=\pi$ ). Because our spectra are extracted from the MC simulation by binning the emerging photon packets, we suffer from the MC noise. In particular, when the flux is smaller, the noise level becomes larger. For example, this is seen in the larger noise level at the longer wavelength that is especially apparent for the maximum spectra, or at the wavelength corresponding to the Ca II NIR absorption especially in the later-phase spectra. If there is a real feature produced by hydrogen above the level of the $\mathrm{MC}$ noise, the ratio at the corresponding wavelength should show an imbalance with respect to unity (or zero in the logarithmic scale); that is, the emission appears as the increase in the ratio (above unity), and the absorption appears as the decrease in the ratio (below unity). As such, a P-Cygni profile in the flux spectra should also appear in the same way in the ratio spectra shown in Figures 15-17.

The $\mathrm{H}_{\alpha}$ emission is seen in these figures (above the MC noise). Irrespective of the epoch, the ratio at the $\mathrm{H}_{\alpha}$ is at the $10 \%$ level 

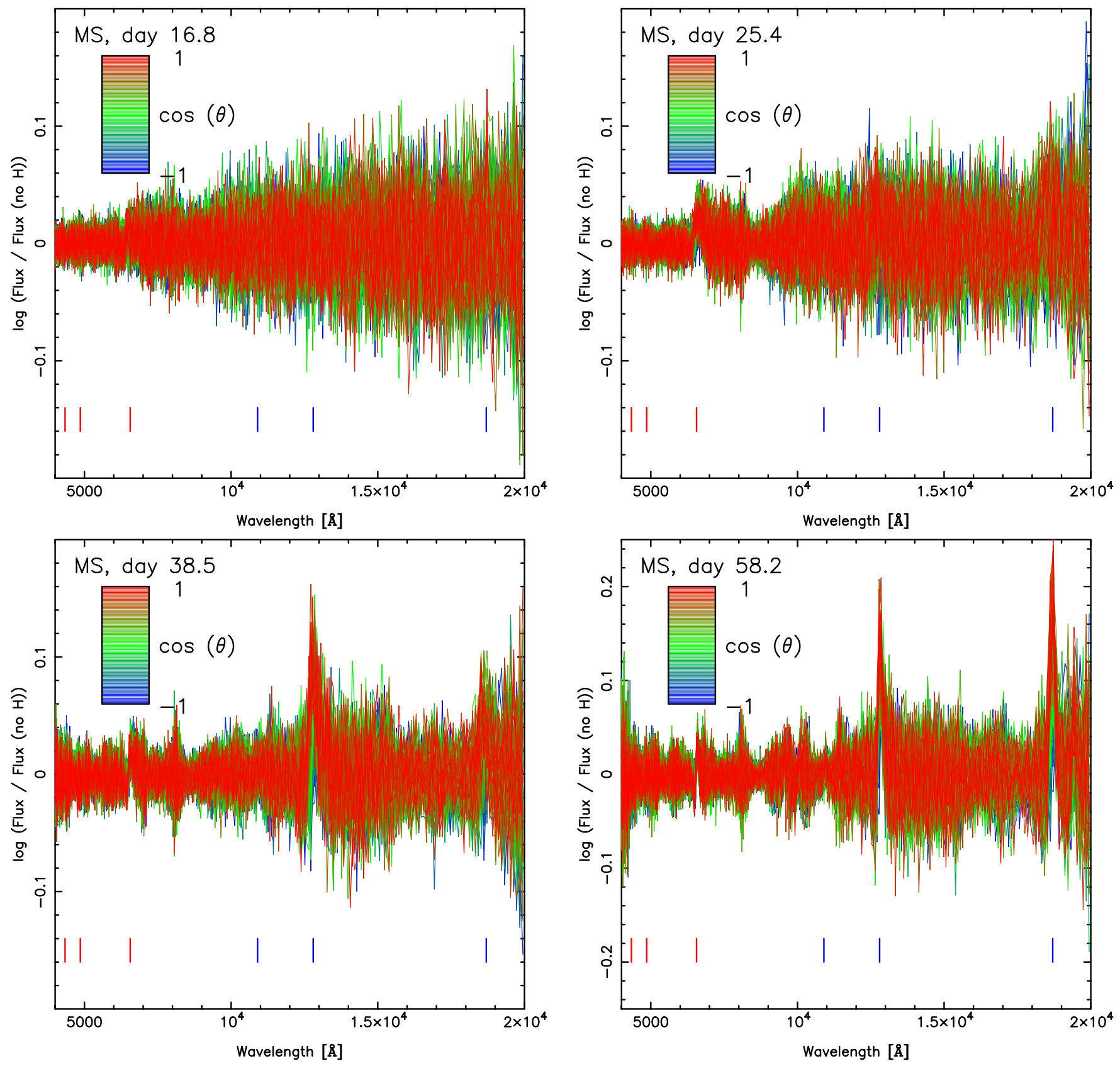

Figure 17. Ratio of the spectral flux with and without hydrogen lines for Model MS.

(A color version of this figure is available in the online journal.)

(but note that this is probably an overestimate for Model MS). That is, if the observed signal-to-noise ratio exceeds this level and if one knows the hydrogen-free reference spectrum a priori, one may detect hydrogen through $\mathrm{H}_{\alpha}$. We thus confirmed the suggestion by Kutsuna \& Shigeyama (2013), although they did not discuss the latter condition. We investigate this issue later in this section.

We find from these figures that the hydrogen lines in the NIR could provide potentially much stronger signals than $\mathrm{H}_{\alpha}$. Around the $B$-band maximum brightness, strengths of $\mathrm{P}_{\alpha}$ and $\mathrm{P}_{\beta}$ are below the noise level of our MC simulation in the corresponding wavelength $(\sim 20 \%$ level $)$. As time goes by, the signal becomes stronger and exceeds the MC noise. At $\sim 25$ days after the explosion (i.e., about a week after the $B$-band maximum), the $\mathrm{P}_{\alpha}$ and $\mathrm{P}_{\beta}$ signals are at about the $25 \%$ level. Later on at $\sim 39$ and 58 days after the explosion (about three and six weeks after the $B$-band maximum), the signals reach the 50\%-60\% level. This behavior is seen in all of our models (although it might be overestimated for the MS model). Note that while the epochs mentioned above are about one month after the $B$-band maximum, these are around (or just a bit later than) the NIR second-maximum date, i.e., much earlier than the previously proposed test for $\mathrm{H}_{\alpha}$ emission in the late phase (about a year after the explosion).

The $\mathrm{H}_{\alpha}$ emission is indeed almost invisible to the eye at this flux level (Figure 10). In this sense, $\mathrm{P}_{\beta}$ is more promising. Figure 18 shows that this feature could be visible even by visual inspection. Note that the flux-axis scale in this figure is reduced: 

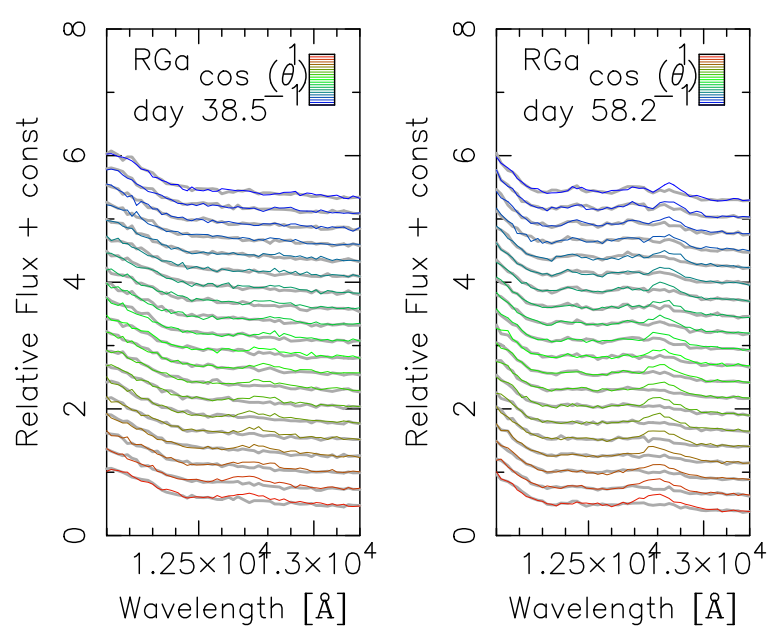

Figure 18. $\mathrm{P}_{\beta}$ in the simulated spectra. In this figure, the spectra for an observer at different directions are added with an additional offset $(\theta=0$ to $\theta=\pi$, from bottom to top). The color curves are the model curves with hydrogen, and the gray curves are without hydrogen. $\mathrm{P}_{\beta}$ is present in these epochs, showing variations in the flux and profile for different viewing directions.

(A color version of this figure is available in the online journal.)

for example, Figure 6 shows $\mathrm{P}_{\beta}$ more clearly. Figure 18 shows that the appearance of this feature is also dependent on the viewing angle. At $\sim 40$ days (around the NIR maximum), the feature is only visible for $\theta=0$. Later on at $\sim 60$ days, the feature is visible for all $\theta$, with the larger flux and broader feature shifted to the blue (i.e., larger velocity) for $\theta=0$.

Figures 19 and 20 show the distribution of the Sobolev optical depths of $\mathrm{H}_{\alpha}$ and $\mathrm{P}_{\beta}$, respectively. Also shown are the underlying photospheres at the $R$ and $J$ bands, respectively. Generally, the underlying optical depth is smaller in NIR than in optical, so the H-rich region appears above the photosphere earlier in the NIR. At day 38.5, hydrogen above the photosphere is mostly recombined (Figure 8), and this explains why $\mathrm{P}_{\beta}$ becomes stronger around this epoch. At this epoch, the photosphere as seen by an observer with the viewing angle of $\theta=\pi$ does not reach this neutral hydrogen region, so at day $38.5, \mathrm{P}_{\beta}$ is visible only for an observer at $\theta=0$. The Sobolev optical depth is higher for $\mathrm{H}_{\alpha}$ than $\mathrm{P}_{\beta}$, and thus the self absorption is more important in $\mathrm{H}_{\alpha}$. This is one reason why $\mathrm{P}_{\beta}$ is stronger than $\mathrm{H}_{\alpha}$.

As we see, in principle one could see hydrogen lines, especially $\mathrm{P}_{\alpha}$ and $\mathrm{P}_{\beta}$, if one knows the hydrogen-free reference spectra a priori. This is observationally a big issue in two respects: (1) one does not know the fraction of SN ejecta with hydrogen (through the interaction) - this is what we aim to investigate; and (2) when a given wavelength region is contaminated by other lines, there could be diversity (by some mechanisms) that is not directly related to the existence of hydrogen. For point (2), even assuming that the observed SNe Ia are all represented by a series of models presented in this paper, the contaminating lines could show diversity arising from different viewing directions; for example, $\mathrm{H}_{\alpha}$ is contaminated by the much stronger $\mathrm{Si}$ line, and this line does show diversity according to the viewing direction within our model (Figures 9 and 10). This happens irrespective of the existence of hydrogen, even though in this model both the asymmetry and hydrogen contamination have the same origin.

If there is a model that would perfectly describe (fit) the SN spectra, one could rely on such model spectra. Unfortunately, this is not the case: non-LTE effects are suggested to become strong in postmaximum spectral formation, especially in the NIR, and so far there are no very good model spectra for it (e.g., Gall et al. 2012). Although the models generally reproduce main features (including our synthetic spectra), the flux ratios of different lines can be strongly dependent on the NLTE treatment. Thus, to confirm the hydrogen lines observationally, one first has to define a reference (or template) spectrum from the observed samples, and then one has to see the diversity of the individual $\mathrm{SN}$ spectra as compared to the reference spectrum. The reference spectrum can be created either from the entire sample or a subset of the sample. For example, if one creates the reference spectrum from $\mathrm{SNe}$ with similar peak luminosity (or light curve width), that would effectively reduce a diversity related to the peak luminosity (i.e., SN Ia spectral features generally correlate with the peak luminosity; Nugent et al. 1995). The remaining diversity may come from a combination of different effects (see, e.g., Maeda et al. 2010, 2011); a strategy to detect hydrogen would be to see whether there is a diversity associated with the wavelengths of the hydrogen lines.

As an experiment, we here follow the same procedure mentioned above to investigate whether the hydrogen lines could be detectable based on Model RGa beyond other sources of diversity. We compare two cases, with and without hydrogen. In each case, we adopt an angle-averaged spectrum as a reference spectrum (at each epoch) and then compute a ratio of a spectrum viewed from a specific direction and the angle-averaged reference spectrum across the wavelength. This corresponds to the ratio of an individual SN spectrum to the reference spectrum, but using the model spectra. Figure 21 shows the results of this experiment for Model RGa with hydrogen (left panels) and without hydrogen (right panels), around the $\mathrm{H}_{\alpha}$ at day 38.5. Figure 22 shows the same but for $\mathrm{P}_{\beta}$.
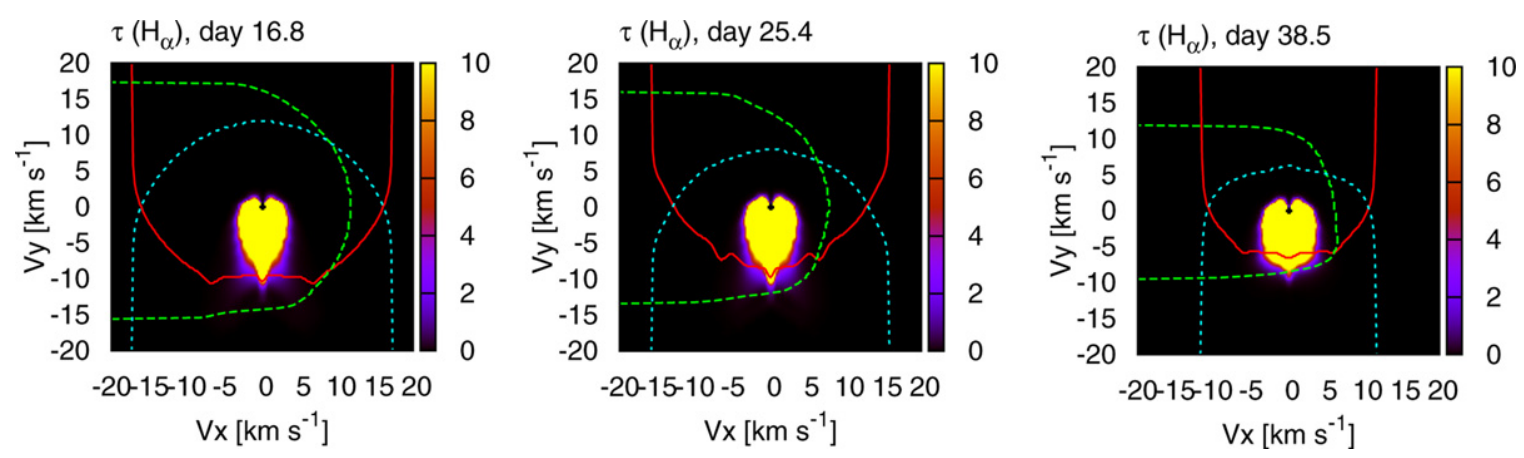

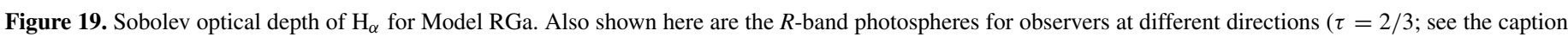
of Figure 7).

(A color version of this figure is available in the online journal.) 

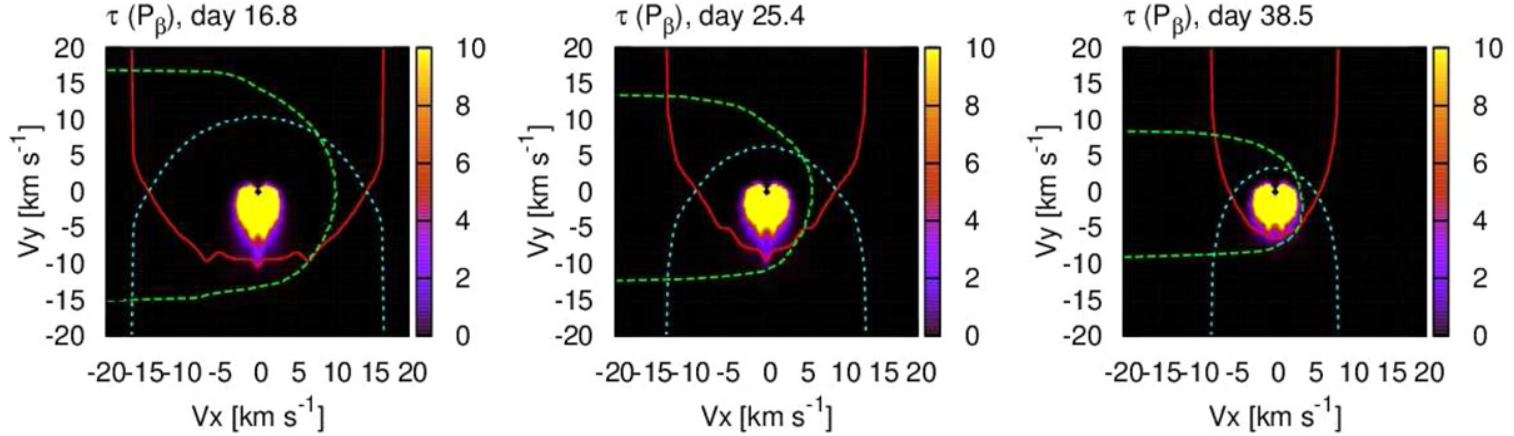

Figure 20. Sobolev optical depth of $\mathrm{P}_{\beta}$ for Model RGa. Also shown here are the $J$-band photospheres for observers at different directions $(\tau=2 / 3$; see the caption of Figure 7).

(A color version of this figure is available in the online journal.)
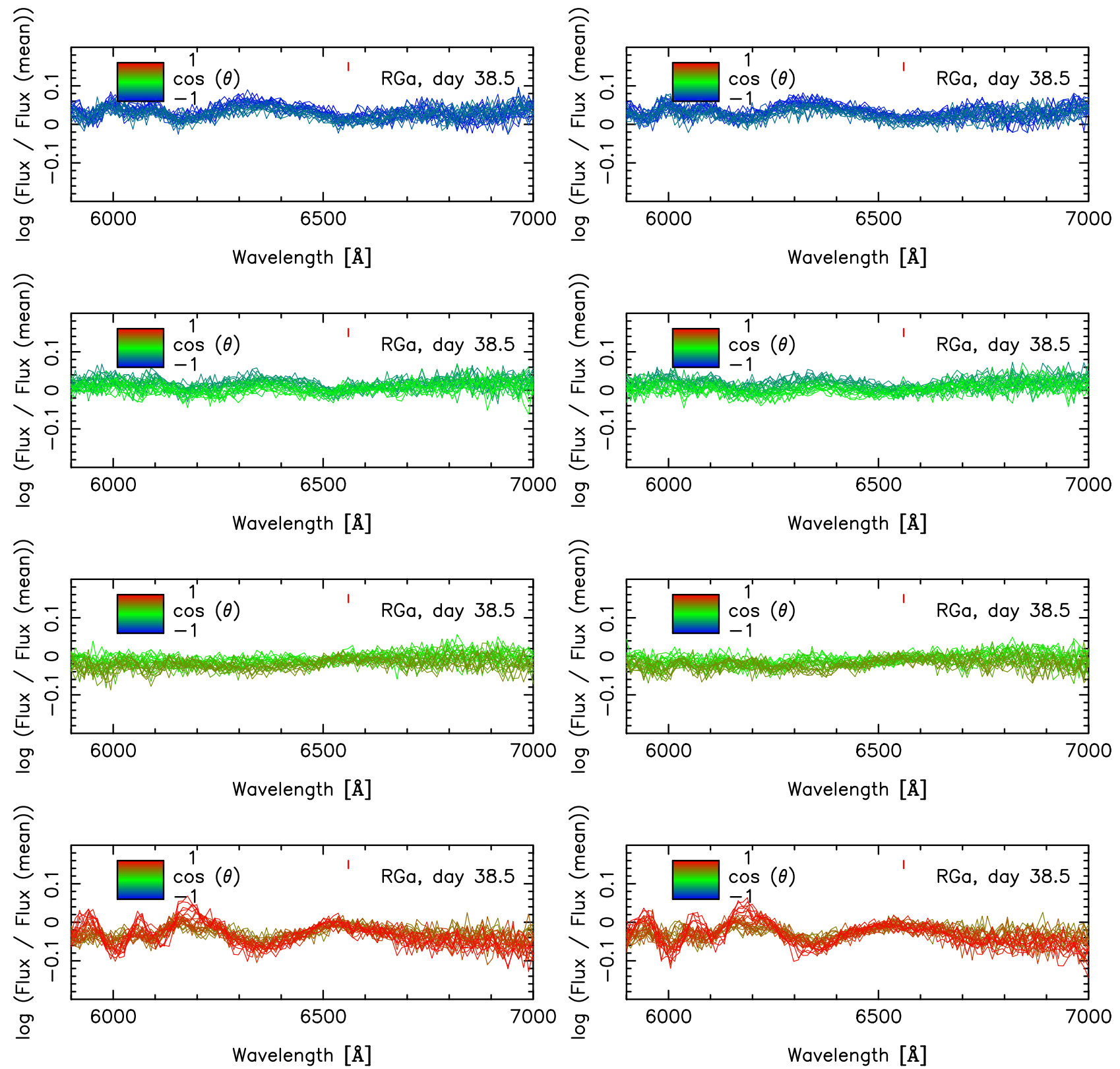

Figure 21. Residuals of the synthetic spectra for Model RGa after being divided by the mean spectrum, shown for the optical range covering Si II 6355 and $\mathrm{H}_{\alpha}$. The left panels are for the original model, and the right panels are for spectra artificially removing the hydrogen transitions. The panels are divided into four parts according to the viewing direction $(\theta=0$ to $\theta=\pi$, from bottom to top). The synthetic spectra are binned within three time bins, but no additional binning is performed in the wavelength and viewing angle directions. The left and right panels are almost identical, showing that it is not observationally feasible to detect $\mathrm{H}_{\alpha}$ at this epoch. Alternatively, the signature of overall ejecta asymmetry could be probed.

(A color version of this figure is available in the online journal.) 

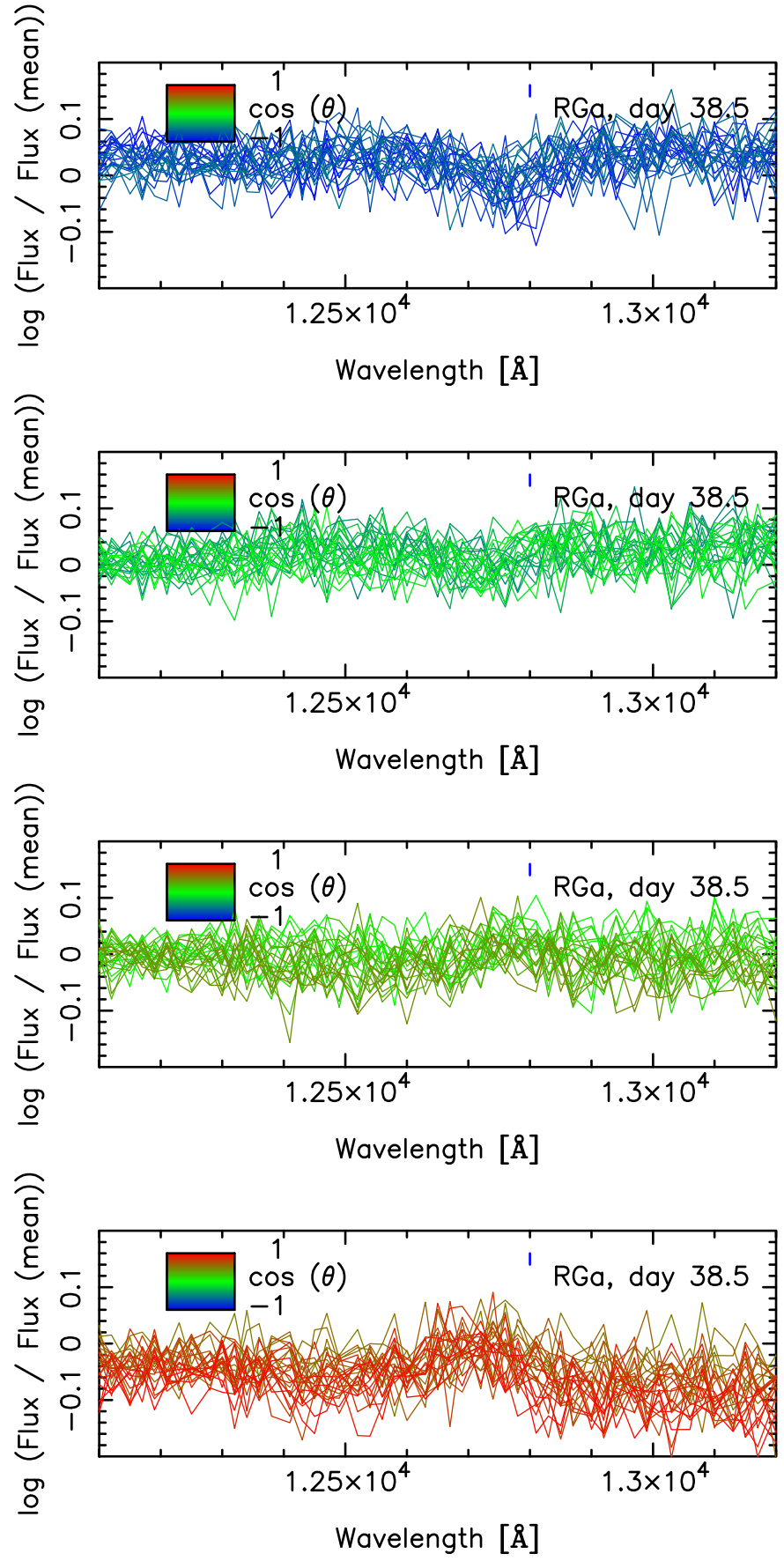
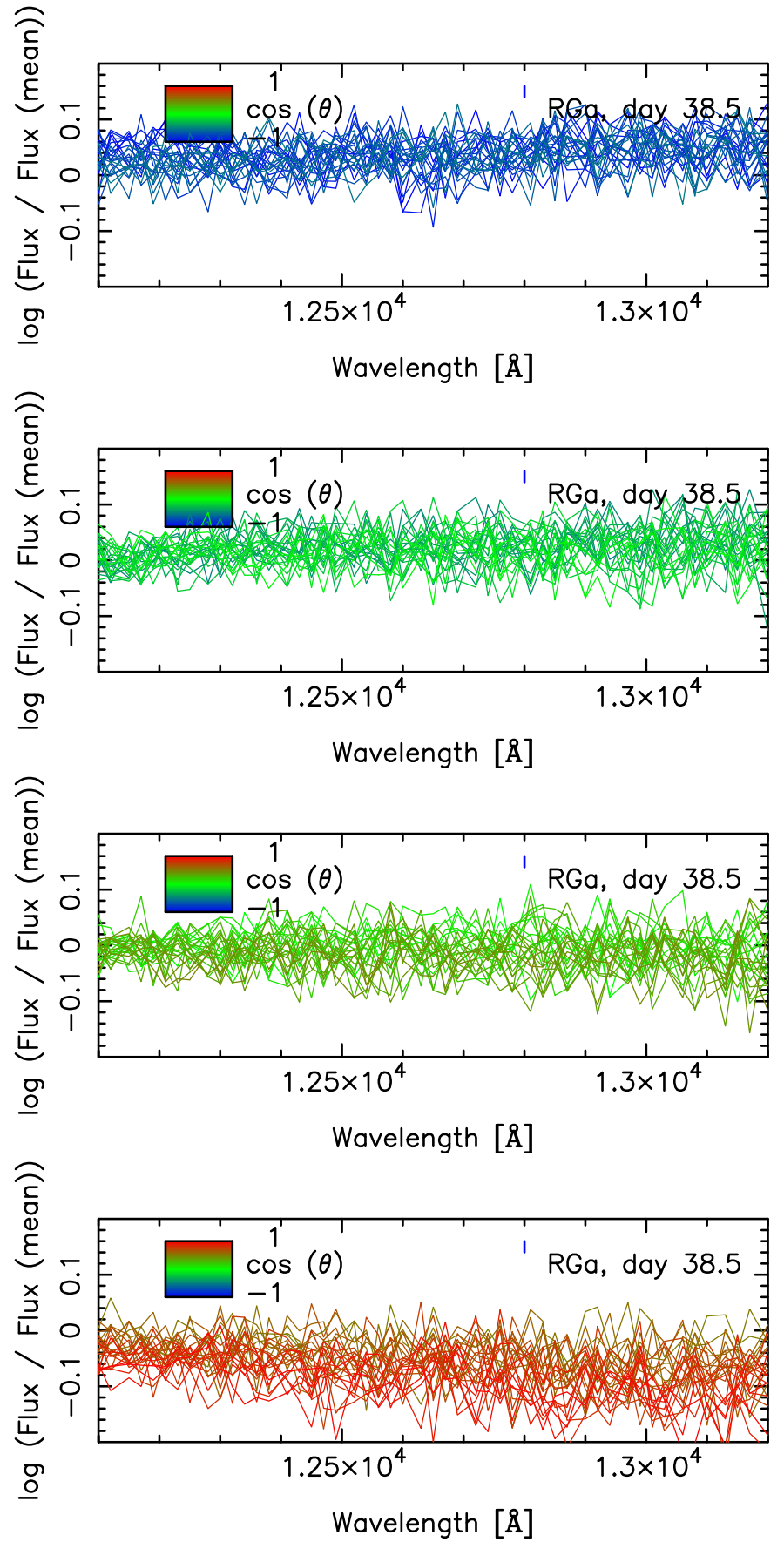

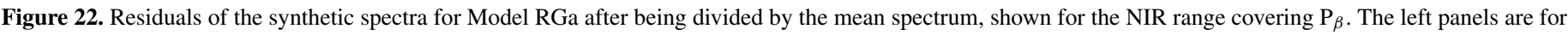

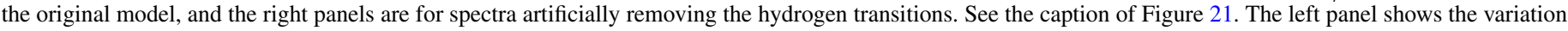

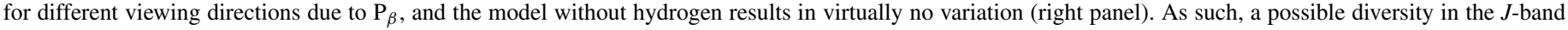
spectra in many SN samples could be used to investigate the presence of hydrogen and a companion.

(A color version of this figure is available in the online journal.)

In general, the optical range shows a larger diversity than NIR due to the viewing direction difference. This highlights more complicated spectrum formation in optical (due to many overlapping lines) than in NIR, at least at this epoch. This makes the identification of $\mathrm{H}_{\alpha}$ quite difficult: the diversity patterns with and without hydrogen are virtually identical, so one cannot practically identify $\mathrm{H}_{\alpha}$. In contrast, $\mathrm{P}_{\beta}$ shows a clear feature at its characteristic wavelength for the hydrogen-contaminated model spectra; the model predicts that either absorption or emission could be seen at the wavelength of $\mathrm{P}_{\beta}$, depending on the viewing direction. This feature is not seen in the case of the hydrogenfree model spectra. Thus, our model predicts that the diversity at the wavelength of $\mathrm{P}_{\beta}$ should arise for Model RGa, and for individual $\mathrm{SNe}$ it can either appear as emission or absorption, depending on the viewing direction, when compared with a mean template spectrum.

Although NIR spectra at this epoch are still rare, the quick development of NIR detectors and increasing opportunities in NIR observations (Marion et al. 2006, 2009) make it an appealing and potentially powerful diagnostic. For example, 
the magnitude of SN Ia 2003du was about $R \sim 20$ at 200 days after the explosion and $R \sim 22$ at one year after the explosion. It was $J \sim 16$ at about two weeks after the $B$-band maximum (Stanishev et al. 2007), and it must have been even brighter in $J$ at about one month after the $B$-band maximum if we apply the Hsiao template light curve (Hsiao et al. 2007; see also Appendix A). Thus, the observational requirements for the postmaximum $\mathrm{P}_{\beta}$ diagnostics may well be less tight than that for the late-time $\mathrm{H}_{\alpha}$ diagnostics in terms of the baseline sensitivity in different band passes.

\section{CONCLUSIONS AND DISCUSSION}

In this paper, we have investigated possible observational signatures of a nondegenerate companion star in the progenitor systems of SNe Ia. Based on hydrodynamic simulations of the impact between expanding SN ejecta and the companion star, we have performed detailed radiation transfer simulations. We have focused on the maximum and postmaximum phases (covering the first two months). While the best data set of SNe Ia is available for these phases, the issue has been until now investigated mostly for the very early phase and late phase, for which the observations are more challenging.

Compared to a previous study by Kasen et al. (2004), our approach is different in the following aspects: (1) we start with the hydrodynamic simulations rather than assuming a simplified kinetic model, (2) we follow the temporal evolution, and (3) we analyze not only the optical properties but also the NIR, with an extended analysis of various observationally testable features. Because of the differences, our model predictions indeed differ from those by Kasen et al. (2004) even qualitatively.

We have found that the overall properties, especially photometric ones, are not much different between the systems with and without a companion star, even with the RG companion. Interestingly, we find in our simulations that the light curves seen from the companion side are not bluer and brighter as suggested in the previous study (Kasen et al. 2004); we predict the opposite. The difference is however generally at a $0.1 \mathrm{mag}$ level. Therefore, the existence of a nondegenerate companion star is not ruled out for individual SNe by the currently available maximum and postmaximum data of SNe Ia. The model predicts a diversity arising from different viewing angles (at the level of $0.1 \mathrm{mag}$ ), showing some correlations between different colors and magnitudes. In the optical wavelength band, interestingly the expected relations are similar to those introduced by an external extinction, whose nature is yet to be clarified. This indicates that this effect, if the progenitor with a nondegenerate companion explains a good fraction of observed SNe Ia, can introduce systematic errors at the level of $0.1 \mathrm{mag}$ when using SNe Ia as standard candles. We have found that the NIR properties do not follow the external-extinction properties, highlighting the importance of NIR observations in developing the SN Ia luminosity/distance calibration better than the 0.1 mag level.

The difference between models with and without a companion is bigger in spectroscopic features than in photometric features. We predict that the Si II 6355 velocity (and other lines) depends on the viewing direction. At the maximum brightness, the Si II 6355 velocity is smaller for an observer viewing from the companion side $(\theta=0)$, as is consistent with the result by Kasen et al. (2004). The temporal evolution of the feature shows an even more interesting behavior. Before the maximum, the Si II feature does not show a strong viewing angle dependence.
Later on, from just before the maximum date, the Si II velocity starts decreasing quickly for an observer viewing from the companion side. The predicted relations between the velocity and the optical $(B-V)$ color, as well as the velocity and the velocity gradient, are found to be different from those inferred from the observations. Thus, the angle variation on the companion-induced asymmetry cannot be a source of these relations. Indeed, we do not try to explain the relations but alternatively suggest using these relations to constrain the existence of a nondegenerate companion star. There should be other mechanisms (e.g., the mass of ${ }^{56} \mathrm{Ni}$ and other factors) that introduce the observed diversity/relation, so the variation due to the viewing angle based on the present model should be regarded as the minimum variation. Therefore, the predicted variations should not be larger than the observational variations. If this is violated, it means that such a model does not account for the bulk of the observed SNe Ia. Comparing the predicted variations with the observed scatters in the velocity-color and velocity-velocity gradient relations, we have found that the model is marginally consistent with the current observations. In the future, observations with better calibrations (especially in photometry) are expected to place a strong constraint on the existence of a nondegenerate companion star from this aspect.

We have also investigated whether there is a chance to probe the nondegenerate companion through the hydrogen features in maximum and postmaximum spectra. We confirmed the expectation (not quantitatively shown before) that $\mathrm{H}_{\alpha}$ is difficult (or practically impossible) to detect in these phases. Alternatively, we suggest that $\mathrm{P}_{\beta}$ can potentially be used as a diagnostic around the NIR maximum phase (or slightly later). We have shown that detecting this feature is observationally feasible and can be even easier than the search for the $\mathrm{H}_{\alpha}$ emission in the later phases.

As a demonstration of the observational feasibility, in Figure 23 we show a comparison between NIR spectra of two SNe Ia, 1999ee (Hamuy et al. 2002) and 2005cf (Gall et al. 2012), which have published NIR data at similar epochs. The data were obtained through the Weizmann Interactive Supernova Data Repository (Yaron \& Gal-Yam 2012). The comparison shows that these two SNe are extremely similar in the NIR, and this similarity in the NIR provides an ideal situation to investigate particular features (in this case $\mathrm{P}_{\beta}$ ) because defining the continuum (or template) is relatively straightforward. There is indeed a hint of the developing difference around $\mathrm{P}_{\beta}$ between the two $\mathrm{SNe}$ in the later phase ( $\sim 40$ days after the $B$-band maximum), although one has to carefully check the data reduction process to confirm that it is not an artifact. This is beyond the scope of this paper, and we will examine a sample of NIR data in a separate paper (K. Maeda, in preparation).

Here as a demonstration we use the spectra of SN 1999ee as templates and investigate the constraint on the amount of hydrogen contaminated in the ejecta of SN 2005cf. Figure 23 also shows the $\mathrm{P}_{\beta}$ at the corresponding epochs, shown in the bottom of both panels, extracted from Model RGa for $\theta=0$ (red) and $\pi$ (blue). This flux is then added to the original (observed) spectrum of SN 1999ee. This way, we can check whether the contamination of the H-rich materials in SN $2005 \mathrm{cf}$ is consistent with the model, assuming that there is no contaminated H-rich material in SN 1999ee. Further, by varying the model flux, we can place a constraint on the amount of $\mathrm{H}$ allowed for SN $2005 \mathrm{cf}$.

At $\sim 30$ days after the $B$-band maximum, Model RGa predicts that $\mathrm{P}_{\beta}$ is visible if viewed from the companion side $(\theta \sim 0)$ 

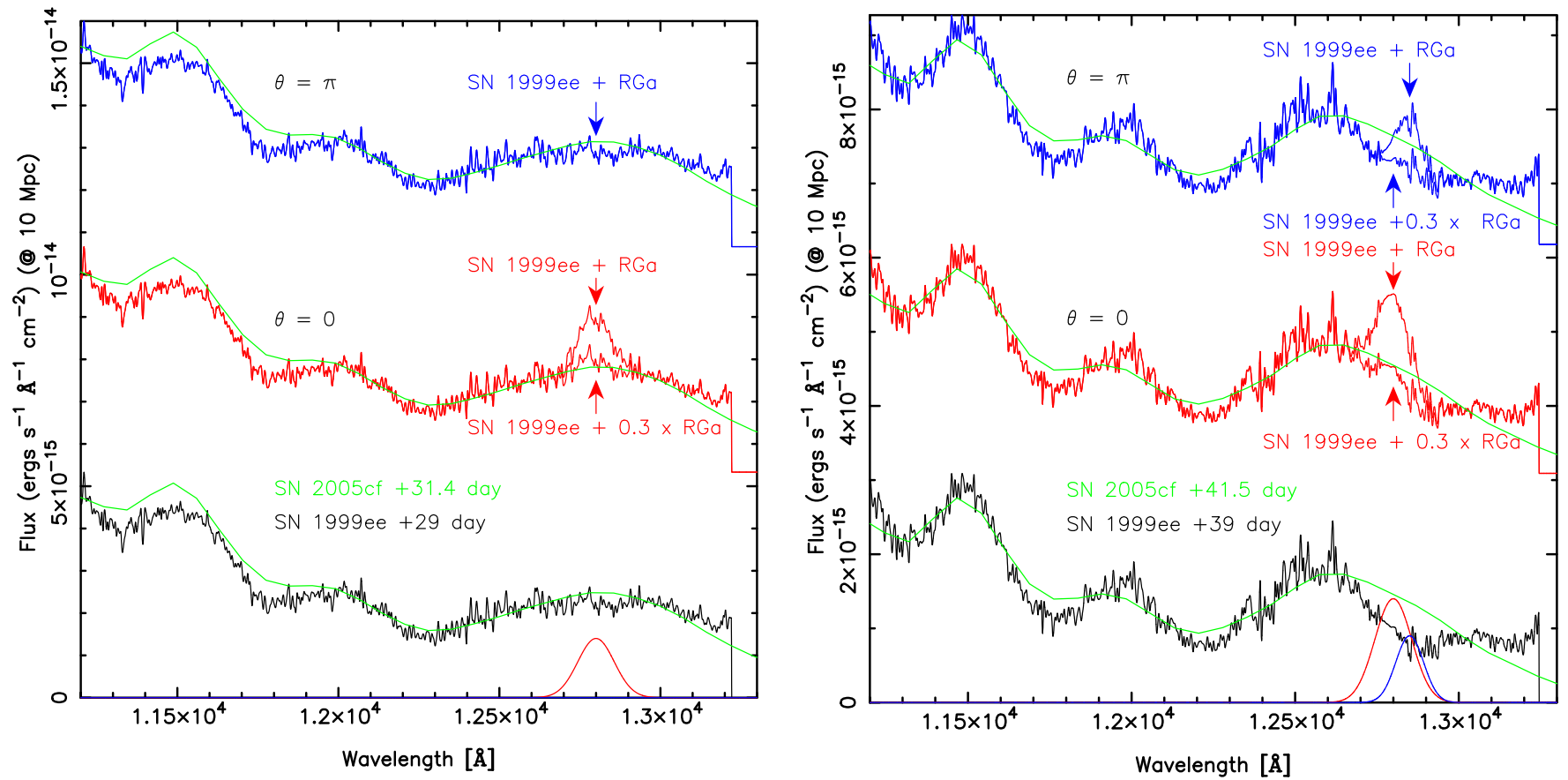

Figure 23. Observed $J$-band spectra (bottom) of SNe 1999ee (black) and $2005 \mathrm{cf}$ (green) at $\sim 30$ days after the $B$-band maximum (left) and $\sim 40$ days (right). The flux of SN $2005 \mathrm{cf}$ is brought to the hypothesized distance of $10 \mathrm{Mpc}$, assuming the original distance of $28 \mathrm{Mpc}$. The flux of SN 1999ee is scaled to roughly fit the flux of $2005 \mathrm{cf}$ at a similar epoch in the $J$ band. The $\mathrm{P}_{\beta}$ line in the synthetic spectra is approximated by a Gaussian profile, extracted from Model RGa (bottom, red for $\theta=0$ and blue for $\theta=\pi$ ). This is added to the spectrum of SN 1999ee for an observer at $\theta=0$ (middle) and at $\theta=\pi$ (top). In doing this, two cases are shown (see the labels in the figure): one with the original prediction and one where the synthetic $\mathrm{P}_{\beta}$ flux is multiplied by 0.3 (roughly corresponding to the $\mathrm{H}$-rich envelope mass scaled down to $0.1 M_{\odot}$ ). This kind of analysis could be used to constrain the amount of stripped-off hydrogen and thus the existence of a companion star (see the text for details).

(A color version of this figure is available in the online journal.)

but not so from the opposite side $(\theta \sim \pi)$. Adding the $\mathrm{P}_{\beta}$ flux predicted by Model RGa for an observer at $\theta=0$ to the spectrum of SN 1999ee, this exceeds the observed flux of SN $2005 \mathrm{cf}$ at that wavelength. Thus, a situation in which SN 2005cf had an RG companion in the close binary system and it was viewed from the companion side is ruled out. If one reduces the predicted $\mathrm{P}_{\beta}$ flux to $30 \%$ of the original (corresponding to $\sim 0.1 M_{\odot}$ of the mixed hydrogen), this would not conflict with the observation. Any companion star is not ruled out for an observer at $\theta=\pi$. Later on at $\sim 40$ days after the $B$-band maximum, a similar but tighter constraint can be obtained: $M(\mathrm{H}) \lesssim 0.1 M_{\odot}$ for an observer at $\theta=0$ and $\lesssim 0.2 M_{\odot}$ for $\theta=\pi$. Note that the SN ejecta contaminated with $0.1 M_{\odot}$ of hydrogen can be a typical feature of the SN ejecta-companion impact (see, e.g., Liu et al. 2012), so the diagnostics we propose here can be quite powerful to identify or rule out a nondegenerate companion star.

Note also that by comparing two $\mathrm{SNe}$, we indeed constrain the difference in the hydrogen content in these two SNe. Thus, it is necessary to construct a hydrogen-free template spectrum from a large sample. In doing this, there are several possibilities in the template construction. Dividing the SN sample into subgroups with different peak luminosities (or decline rate) is an obvious choice. One would also be tempted to divide the sample according to the host types or environment properties, then compare the templates for each group as well as compare individual SNe with the templates. Such a strategy may pick up possible different populations in different environments (e.g., Wang et al. 2013), providing a test of how different populations may be related to the SD and DD scenarios.

There are a few limitations in the present study. We have adopted the expansion opacity formalism and the two-level atom approximation rather than simulating the full details of the fluorescence following the excitations. This is a good approximation for ions with complicated level structures like Fepeaks because the high rate of the radiation-matter interactions should establish a quasi-equilibrium that is represented by thermal redistribution (see, e.g., Section 3.6 of Kasen et al. 2006). The use of this prescription for a simple atom like hydrogen may need further justification and calibration.

Another issue is the NLTE effects. For SNe Ia, the NLTE effect on ionization is especially strong at $\gtrsim 50$ days after the explosion, but it is not so in the earlier phase (Kromer \& Sim 2009). Around the peak date, a strong effect can be seen in the $\mathrm{UV}$, but the effect is not significant in the optical range if an appropriate value for the thermalization parameter is adopted (Baron et al. 1996). For SNe IIp, which could be relevant to our investigation of the hydrogen lines, deviations from LTE have significant effects on line profiles, but the continuum flux is not much affected (Baron et al. 1996; Dessart \& Hiller 2008; Kasen \& Woosley 2009) (see, e.g., Figure 3 of Baron et al. (1996)). Indeed, a bigger effect is expected for time-dependent NLTE effects, which for SNe IIp models could introduce a change in the flux level of $\mathrm{H}_{\alpha}$ by a factor of a few (Dessart \& Hiller 2008). Still, this effect would not remove all of the $\mathrm{H}_{\alpha}$ flux predicted in the LTE calculations (Dessart \& Hiller 2008), and thus we would not expect that our results will be changed qualitatively by the NLTE effects.

Related to the NLTE effects, in our formalism the effect of the fluorescence is taken into account by a single thermalization parameter $(\epsilon)$, and it has been shown that the biggest difference from the present prescription $(\epsilon=0.3)$ is expected for the pure scattering atmosphere $(\epsilon=0)$ (Baron et al. 1996; Kasen et al. 2006). To check whether this particular choice of $\epsilon$ (calibrated for metal lines) would affect the strength of hydrogen features, 
we repeated the same calculations for Model RGa but setting $\epsilon=0$. The result is shown in Appendix C. We conclude that this does not introduce much difference. We caution that the two-level approximation and the thermalization parameter cannot be exactly calibrated to a full NLTE description, and therefore introducing the thermalization parameter is merely an approximation. Ultimately it should be tested by the full NLTE calculations for the same models. Still, as mentioned above, the results from the previous studies on SN Ia and IIp models, both relevant to our study, are promising, suggesting that this approximation would not introduce significant errors in the observables of interest in this paper.

We note that neglecting nonthermal excitations of hydrogen by $\gamma$-rays might indeed lead to underestimates of the hydrogen line fluxes. Also, in our model we omit a metal content in the companion envelope by assuming the purely hydrogen and helium in it. This would not change the overall feature because the main part of the emission is created by the SN ejecta and the companion envelope merely dilutes the emission through Thomson scattering. Inclusion of the metal would increase the heating of the H-rich region and therefore would keep the ionization of hydrogen high for a longer time than in our present model. However, as time goes by, the $\gamma$-ray heating, which is not sensitive to the metal content, becomes progressively important. As a result, our prediction of the appearance of $\mathrm{P}_{\beta}$ in relatively late phases would not be dramatically affected by the metal content in the companion envelope.

Besides the hydrogen issue, the ejecta asymmetry is a characteristic feature of a nondegenerate companion system, and we predict that this configuration leads to a characteristic diversity pattern across the wavelengths (Figures 21 and 22). In Appendix D we show the expected diversity patterns at different epochs for Model RGa. In the same way as we propose for searching for $\mathrm{P}_{\beta}$, the comparison of a spectrum of an individual $\mathrm{SN}$ and a template spectrum can in principle be used to search for such a diversity pattern. This is another way we propose to search for a signature of nondegenerate companion stars in SN Ia systems.

This research is partly supported by the World Premier International Research Center Initiative (WPI Initiative), MEXT, Japan. K.M. acknowledges financial support by Grant-in-Aid for Scientific Research for Young Scientists (23740141, 26800100). Numerical computations presented in this paper were carried out using 512 cores on a Cray XC30 at the Center for Computational Astrophysics, National Astronomical Observatory of Japan. We thank Ken'ichi Nomoto for providing the W7 model and constructive discussion and Elena Sorokina and Sergei Blinnikov for providing their synthetic light curves for the W7 model. We also thank the anonymous referee for the extensive review and many constructive comments. We used the Weizmann Interactive Supernova Data Repository (www.weizmann.ac.il/astrophysics/wiserep) to obtain the archival spectra data of SNe 1999ee and 2005cf.

\section{APPENDIX A}

\section{METHOD OF RADIATION TRANSFER}

Our radiation transfer simulation code adopts the Monte Carlo method, where paths of individual photon packets are computed as a random walk process. This method is broadly adopted in radiation transfer simulations in SN ejecta (Lucy 2005 and references therein). It is suited to treating Doppler shifts of photons due to the velocity gradient in the expanding/moving medium and the resulting enhancement of the bound-bound opacities or expansion opacities (Karp et al. 1977; Eastman \& Pinto 1993). Because of the successive Doppler shifts in a comoving frame a photon experiences as it flies through the moving medium, it can suffer from the discrete transitions (bound-bound) at frequencies different from the original frequency of a photon at its creation.

We largely adopt the prescriptions given by Lucy (2005), Kasen et al. (2006), and Kromer \& Sim (2009). Our simulation is pure radiation transfer, so the kinetic and composition structure $\left[\boldsymbol{v}(\boldsymbol{r}, t), \rho(\boldsymbol{r}, t), X_{i}(\boldsymbol{r}, t)\right]$ should be provided as a background (i.e., no feedback process from the radiation to hydrodynamics is taken into account). The radiation transfer simulation provides iteratively the thermal and ionization conditions and accordingly the distribution of opacities $\left[T(\boldsymbol{r}, t), n_{i}^{j}(\boldsymbol{r}, t), \alpha_{\lambda}(\boldsymbol{r}, t)\right]$ (where $n_{i}^{j}$ is the number density of an ion $j$ at $i$ th level) so as to be consistent with the radiation field $\left[f_{\lambda}(\boldsymbol{r}, t, \boldsymbol{l})\right]$ (here $\boldsymbol{l}$ is the photon direction vector) under the assumptions of LTE and radiative equilibrium.

The code is applicable to the 1D spherical coordinate, the 2D spherical-polar coordinate, and the 3D spherical-polar and Cartesian coordinates, which can be simply specified in an input parameter file. The $1 \mathrm{D}$ and $2 \mathrm{D}$ versions assume spherical symmetry and axisymmetry so that the number of photon packets can be reduced to reach the convergence. We have performed various test calculations to confirm that the imposed asymmetry does not introduce errors in the transfer simulations (an example is described later).

The multidimensional/frequency/epoch radiation transfer code, HEIMDALL, takes the following steps in simulating the radiation transfer. The main part of the code is written in a general way so that the applicability is not restricted to the radiation transfer in the $\mathrm{SN}$ ejecta but below when necessary specific functions for the $\mathrm{SN}$ radiation transfer are described. The code is written in a hybrid-parallelization mode using openMP and MPI, and its parallelization efficiency has been tested on up to 512 cores distributed over 64 CPUs.

\section{A.1. Determining the Distribution of Initial Photon Packets}

Over the course of the main MC routine, the properties of a photon packet are described by a set of variables $[\boldsymbol{r}(t), \boldsymbol{l}(t), \lambda(t), \varepsilon(t)]$, i.e., the position, direction vector, wavelength, and the total energy within the packet. Here, we describe the photon packet (or an MC quanta) as a group of identical photons (or particles), i.e., $\varepsilon=n_{\mathrm{ph}} h v$ where $n_{\mathrm{ph}}$ is the number of photons in a packet and $v$ is the frequency. To compute the change in these variables as a function of time $(t)$ by the main MC routine, we have to determine the initial conditions, $\left[\boldsymbol{r}\left(t_{0}\right), \boldsymbol{l}\left(t_{0}\right), \lambda\left(t_{0}\right), \varepsilon\left(t_{0}\right)\right]$, where $t_{0}$ is the time of the creation of the thermal photon under consideration.

Photon packets created by processes other than interactions of already existing thermal photons and matter are specified at the beginning of simulations (note that photons created by such interactions between already existing thermal photons and matter are treated over the course of the main MC transfer). For simulations performed in this paper, these are photons created as a result of radioactive decay energy input through the decay chain ${ }^{56} \mathrm{Ni} \rightarrow{ }^{56} \mathrm{Co} \rightarrow{ }^{56} \mathrm{Fe}$. These $\gamma$-ray and positron packets are created at the beginning of a simulation based on the distribution of ${ }^{56} \mathrm{Ni}$ and its decay property the photon packets are assigned with a spectral energy (i.e., branching ratios in the 
decays) and time of creation (i.e., decay time) determined by random number generation. If the time at the creation of a $\gamma$-ray packet, as determined by the MC random number generation for every packet, is earlier than the starting time of the whole simulation, the $\gamma$-ray packet is assumed to be absorbed by the simulation start time at the position of the creation in the comoving frame. This deposited energy is converted to optical photons at the starting time of the optical photon transfer, taking into account the adiabatic loss of the thermal energy between the deposition time and the simulation starting time, assuming that the optical photons created here have diffused negligibly to matter in this time interval (Lucy 2005; Kromer \& Sim 2009). This treatment is justified by the short mean free paths of photons in the early phase, but the approximation becomes less robust if the starting time for the transfer simulation is taken to be later. Our simulations are started at 10 days after the explosion (approximately a week before the $B$-band maximum), and we have checked the applicability of this approximation in Appendix B, where we find that our results are not sensitive to this relatively late starting time of the simulations. Transfer of $\gamma$-rays is solved by taking into account Compton scattering, pair creation, and photoelectric absorption based on the scheme identical to optical photons but without temperature iteration because the cross sections of these interactions are insensitive to the thermal condition. Positrons are assumed to deposit their energy in situ. The details of the computational method here are given by Maeda (2006). During the MC transfer simulation, the energy deposition by $\gamma$-rays and positrons, $\Gamma_{\gamma}(\boldsymbol{r}, t)$, is tracked in the same manner as with the heating by UV or optical photons (see below).

Because the energy deposition by $\gamma$-rays and positrons is the only source of the thermal energy (i.e., ultimately the energy of the thermal radiation) in the present situation, the transfer simulation described above provides the initial condition for the thermal photon packets to be followed by the main MC routines. With the energy deposition rate $\Gamma_{\gamma}(\boldsymbol{r}, t)$ we thus determine the total energy content of the thermal photon packets emitted at a given spatial bin and time bin. In the calculations shown in this paper, the energy content of each thermal photon packet $(\varepsilon)$ is set to be equal for all of the packets at its creation. We first integrate the energy deposition rate in space and time, and then the number of thermal photons created at a given spatial bin and a given time bin is determined by the relative contribution of $\Gamma_{\gamma}(\boldsymbol{r}, t)$ (as integrated within a spatial bin and time bin) to the total deposited energy. The position and time at its creation within the spatial and time bins are determined by random number generation (in the comoving frame). The direction vector of the packet is also computed by random number generation assuming the isotropic emission in the comoving frame. Now we have a set of variables to specify the properties of the photon packets in the comoving frame, except for its wavelength, and these are transformed to the $\mathrm{SN}$ rest (or observer) frame. The wavelength, $\lambda\left(t_{0}\right)$, cannot be specified at this step because it requires that the temperature be known. Thus, the creation of the photon packet is coupled with the main MC routine and iteratively solved following the steps described below, to be self-consistent with the ejecta temperature.

\section{A.2. Computing Thermal and Ionization Structures and Opacity Distributions}

At a given time and for a given temperature $T(\boldsymbol{r}, t)$, the ionization and level populations are computed under the LTE assumption, i.e., through the Saha equation and the Boltzmann distribution. Then the opacities are computed as a function of wavelength, including bound-bound, bound-free, free-free, and electron scattering. The electron scattering opacity is computed with the electron number density, $n_{\mathrm{e}}(\boldsymbol{r}, t)$, which is given by the ionization condition:

$$
\alpha_{\mathrm{e}}(\boldsymbol{r}, t)=\sigma_{\mathrm{T}} n_{\mathrm{e}}(\boldsymbol{r}, t),
$$

where $\sigma_{\mathrm{T}}$ is the Thomson cross section. The free-free absorption opacity is computed as follows:

$$
\begin{aligned}
\alpha_{\mathrm{ff}}(\boldsymbol{r}, t, v)= & \frac{4 e^{6}}{3 m_{e} h c}\left(\frac{2 \pi}{3 k m_{e}}\right)^{1 / 2} T(\boldsymbol{r}, t)^{-1 / 2} \sum_{j} Z_{j}^{2} n_{\mathrm{e}} n^{j} v^{-3} \\
& \times\left[1-\exp \left(\frac{h v}{k T}\right)\right] g_{\mathrm{ff}},
\end{aligned}
$$

where $Z_{j}$ is the number of free electrons associated with the ion $j$, and $n^{j}$ is the number density of the ion $j$. The Gaunt factor $\left(g_{\mathrm{ff}}\right)$ is set to be unity. For the bound-free transitions, cross sections $\left[\alpha_{\mathrm{ff}}(\boldsymbol{r}, t, \lambda)\right]$ are taken from Verner \& Yakovlev (1995) and Verner et al. (1996). For a given $\lambda$, the bound-free cross sections are summed over different ions (with the ionization states determined by the Saha equation).

The bound-bound transitions are treated in the Sobolev approximation, where the line optical depth is given as follows:

$$
\tau_{l u}(\boldsymbol{r}, t)=\frac{\pi e^{2}}{m_{e} c} f_{l u} \lambda_{l u} t n_{l}(\boldsymbol{r}, t)\left[1-\frac{g_{l} n_{u}(\boldsymbol{r}, t)}{g_{u} n_{l}(\boldsymbol{r}, t)}\right],
$$

where subscripts $l$ and $u$ denote the lower and upper levels of a transition under consideration (here we omit the superscript $j$ to specify the ion); $f_{l u}$ and $\lambda_{l u}$ are the oscillator strength and the wavelength of the transition; and $g_{l}$ and $g_{u}$ are statistical weights of the lower and upper level, respectively. For the line list, we adopt a standard set of $\sim 5 \times 10^{5}$ bound-bound transitions from Kurucz \& Bell (1995).

With the Sobolev optical depth, the escape probability of the photon out of the resonance region is

$$
\beta_{l u}(\boldsymbol{r}, t)=\frac{1-e^{-\tau_{l u}(\boldsymbol{r}, t)}}{\tau_{l u}(\boldsymbol{r}, t)} .
$$

We treat the bound-bound transitions within the expansion opacity formalism, i.e., we combine the transitions into a discrete frequency grid (Karp et al. 1977; Eastman \& Pinto 1993). Here, the total cross section at wavelength $\lambda$ is given as

$$
\alpha_{\mathrm{bb}}(\boldsymbol{r}, t, \lambda)=\frac{1}{c t} \sum_{l, u} \frac{\lambda_{l u}}{\Delta \lambda}\left(1-e^{-\tau_{l u}}\right)
$$

where the sum runs over the bound-bound transitions whose energy difference is within the wavelength bin under consideration $(\Delta \lambda)$. The purely absorptive component is defined within the two-level atom approximation, i.e.,

$$
S_{\lambda}=\left(1-\epsilon_{l u}\right) J_{\lambda}+\epsilon_{l u} B_{\lambda}(T),
$$

where the source function is divided into the scattering component (i.e., treated as a resonance line) and into the absorptive component (i.e., thermalized after multiple scatterings and fluorescence). Generally, this treatment of the bound-bound transitions is shown to provide a good approximation for the thermal conditions appropriate to $\mathrm{SNe}$ Ia because the large opacities 
and many transitions lead to the thermal redistribution. This has been calibrated with a more detailed transfer where the fluorescence is directly treated (Baron et al. 1996; Kasen et al. 2006). While $\epsilon_{l u}$ is dependent on different transitions, the result of the radiation transfer is indeed insensitive to the exact value of $\epsilon_{l u}$ as long as $\epsilon_{l u} \sim 1$ (see, e.g., Figure 10 of Kasen et al. 2006). For this reason, we adopt the same value for all of the transitions, $\epsilon \equiv \epsilon_{l u}=0.3$ as our standard case, following Kasen et al. (2006). We do caution that the argument is dependent on the focus and topics under investigation. For example, the direct treatment of the fluorescence is essential in the late-time spectral formation (Kromer \& Sim 2009). Now, the explicit form for the purely absorptive component in the bound-bound transitions can be written as follows:

$$
\alpha_{\mathrm{bb}, \mathrm{abs}}(\boldsymbol{r}, t, \lambda)=\frac{1}{c t} \sum_{l, u} \frac{\lambda_{l u}}{\Delta \lambda} \frac{\epsilon_{l u}}{\beta_{l u}+\epsilon_{l u}\left(1-\beta_{l u}\right)}\left(1-e^{-\tau_{l u}}\right) .
$$

The total opacity is given as the sum of the different components described above:

$$
\alpha(\boldsymbol{r}, t, \lambda)=\alpha_{\mathrm{e}}(\boldsymbol{r}, t)+\alpha_{\mathrm{ff}}(\boldsymbol{r}, t, \lambda)+\alpha_{\mathrm{bf}}(\boldsymbol{r}, t, \lambda)+\alpha_{\mathrm{bb}}(\boldsymbol{r}, t, \lambda) .
$$

The purely absorptive component is defined as follows:

$$
\alpha_{\mathrm{abs}}(\boldsymbol{r}, t, \lambda)=\alpha_{\mathrm{ff}}(\boldsymbol{r}, t, \lambda)+\alpha_{\mathrm{bf}}(\boldsymbol{r}, t, \lambda)+\alpha_{\mathrm{bb}, \mathrm{abs}}(\boldsymbol{r}, t, \lambda) .
$$

\section{A.3. Propagation of Photon Packets through the MC Simulation}

With the background condition including opacity distribution (in space and frequency) now specified, the propagation of photon packets is computed from time $t_{n}$ to $t_{n+1}$. When the photon packet already exists at $t_{n}$ from the previous time step, its path until $t_{n+1}$ or until it escapes out of the ejecta before $t_{n+1}$ is followed by the MC simulation for each photon packet, and this procedure is repeated for all of the photon packets. If the photon packet is created at $t_{0}$ between $t_{n}$ and $t_{n+1}$ (due to the $\gamma$-ray and positron deposition), its path is followed from $t_{0}$.

At each step, photon path lengths for several numerical and physical events are computed, and then the event with the minimal length is adopted as the real event. These events include the following cases: (1) a photon reaches a boundary between the current spatial grid and one of the neighboring grids, (2) the physical time the packet experiences reaches the next time step $\left(t_{n+1}\right),(3)$ the photon comoving spectral frequency is redshifted to come into the next frequency bin, and (4) a photon suffers from either scattering or absorption. This procedure is repeated for all of the photon packets.

Item (3) is specific for the radiation transfer in a moving medium like the $\mathrm{SN}$ ejecta. Because we assume the homologous expansion, the Doppler shift is simply computed as $\Delta \lambda=\lambda v / c$, and this inversely gives the distance the packet travels before suffering from the Doppler shift of the amount $\Delta \lambda$. Item (4) is evaluated through the standard MC formula as follows:

$$
\alpha^{\prime}(\boldsymbol{r}, t, \lambda) \rho(\boldsymbol{r}, t) \delta s^{\prime}=-\ln z,
$$

where $z$ is the random number (between 0 and 1) and $\delta s$ is the path length. Here the prime indicates the rest-frame quantities.

When the packet experiences the interaction, its fate after the interaction is again determined through the random number generation, proportional to cross sections to each event. Specifically, we judge whether this is a scattering or absorption, according to the ratio of $\alpha_{\text {abs }}(\boldsymbol{r}, t, \lambda)$ and $\alpha_{\text {scat }} \equiv \alpha(\boldsymbol{r}, t, \lambda)-\alpha_{\text {abs }}(\boldsymbol{r}, t, \lambda)$.
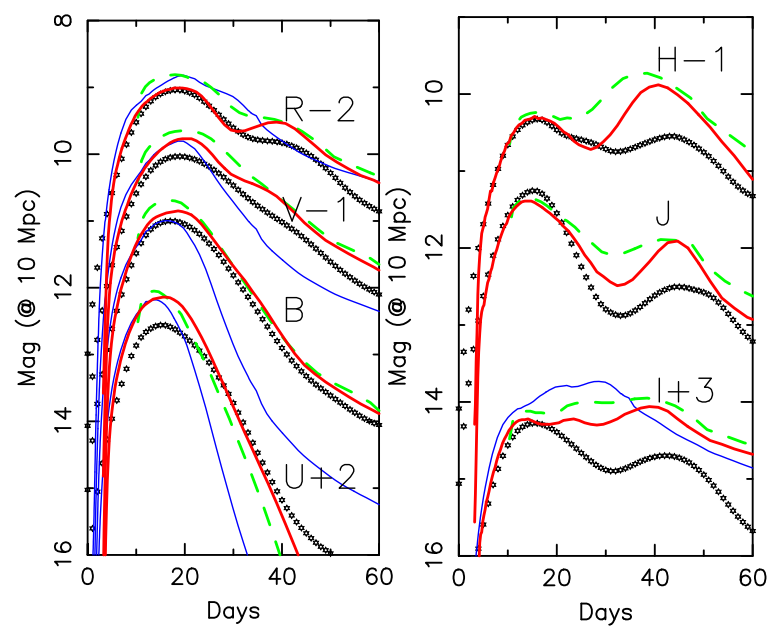

Figure 24. Synthetic multiband light curves as compared with SN Ia template light curves. The SN Ia template light curves (stars) are constructed from the Hsiao spectral template (Hsiao et al. 2007) convolved with standard filter functions. Our synthetic light curves based on the W7 model are shown by red curves (thick solid). For comparison, the synthetic light curves computed by STELLA are shown by blue curves (thin solid), from $U$ to $I$ bands. Our 2D reference W7 model is shown by green curves (thick dashed). For the reference model, we apply an offset of 0.34 mag for all of the bands for a fair comparison because the model has a larger amount of ${ }^{56} \mathrm{Ni}$ (see the text). The amount of the offset here reflects the difference in the mass of ${ }^{56} \mathrm{Ni}\left(0.81 M_{\odot}\right.$ in the reference model and $0.59 M_{\odot}$ in the original W7 model).

(A color version of this figure is available in the online journal.)

A scattering is treated as an isotropic and coherent scattering in the comoving frame, a good approximation for resonance transitions (and Thomson scattering). A new photon direction in the comoving frame is chosen randomly following a standard MC procedure, and it is transferred to the rest frame. It is elastic in the comoving frame, and the transfer from the comoving frame to the rest frame automatically takes into account the adiabatic loss in a microscopic sense.

An absorption and re-emission is treated within the thermalization approximation, and thus a new wavelength at its reemission is determined through the local thermal emissivity:

$$
j_{\lambda}(\boldsymbol{r}, t)=B_{\lambda}(T) \alpha_{\text {abs }}(\boldsymbol{r}, t, \lambda) .
$$

Here the emission is treated as isotropic in the comoving frame.

Note that the propagation is treated in the rest frame, using the cross sections originally computed in the comoving frame but transferred into the rest frame. The physical events (scattering and absorption/re-emission) are treated in the comoving frame, i.e., first the rest-frame quantities are transferred into the comoving frame, computing the outcome in the comoving frame, and then the result is transferred back into the rest frame. The formalisms for the transformation are given by Castor (1972). This way, the Doppler shift in the moving medium is appropriately handled, and for example it results in the P-Cygni profile for bound-bound transitions.

\section{A.4. New Temperature Determination and Iteration for Temperature Convergence}

Over the course of the photon propagation, the heating rate between $t_{n}$ and $t_{n+1}$ at each spatial grid is tracked using the MC estimator:

$$
\Gamma_{\mathrm{opt}}(\boldsymbol{r}, t)=\frac{1}{\Delta t V} \sum_{k} \alpha_{\mathrm{abs}}(\boldsymbol{r}, t, \lambda) \varepsilon_{k} \delta s_{k},
$$




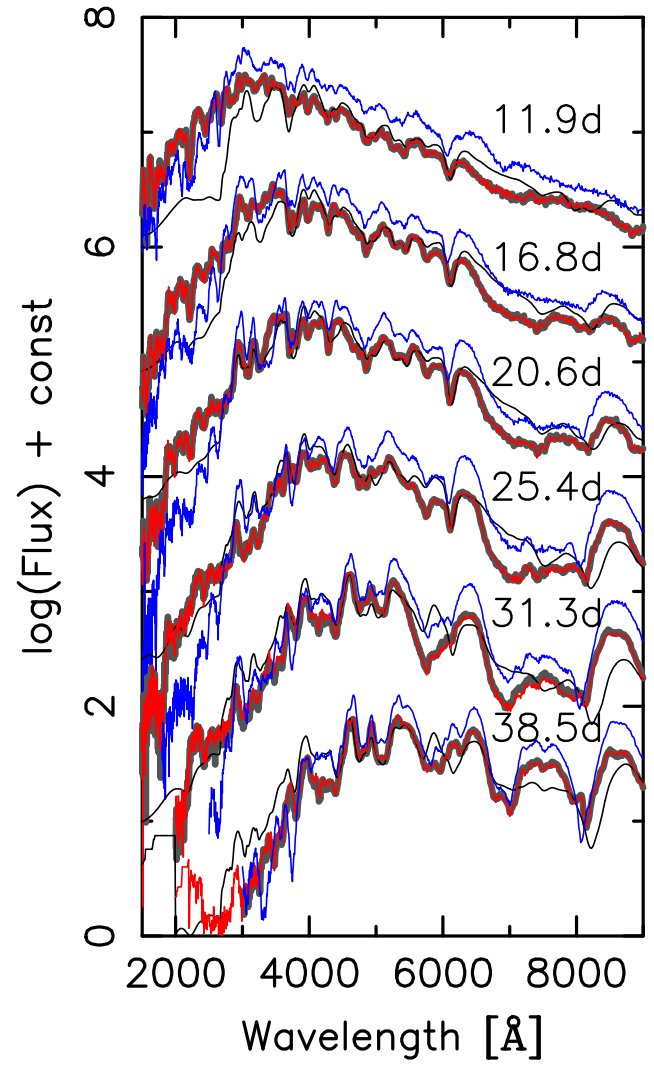

Figure 25. Synthetic spectra as compared with the Hsiao SN Ia template. The zero point in the epoch is the explosion time, and the $B$-maximum date is assumed to be 17 days after the explosion to label the Hsiao template spectra. The W7 model spectra (as computed under the assumption of spherical symmetry) are shown in red, while the 2D reference model spectra are shown in blue. The Hsiao template spectra are shown in black. Synthetic spectra of W7 computed with the radiation transfer scheme in 2D space under the assumption of axisymmetry is shown by (thick) gray lines, to show that our scheme of the radiation transfer in 2D does not introduce any artifacts. The values of the offset applied to each spectrum are the same for all of the models (i.e., no additional offset is applied to provide the best match between the models and the templates). For the spectra at late epochs, a blue portion of the spectra is truncated in the presentation, where the MC noise is large due to the small amount of UV photons in the late phase.

(A color version of this figure is available in the online journal.)

where the quantities are given in the comoving frame, and this estimator runs over all of the packet (as specified by $k$ ), which passes through a given grid between $t_{n}$ and $t_{n+1}\left(\Delta t \equiv t_{n+1}-t_{n}\right)$. Here, $V$ is the volume of the spatial grid. With the heating rate by $\gamma$-rays and positrons obtained in the same manner $\left(\Gamma_{\gamma}\right)$, the heating-cooling balance under the radiative equilibrium provides a constraint on the temperature,

$$
\Lambda(T)=\Gamma_{\mathrm{opt}}(\boldsymbol{r}, t)+\Gamma_{\gamma}(\boldsymbol{r}, t),
$$

where the cooling rate at each spatial grid is given as

$$
\Lambda(T)=4 \pi \int \alpha_{\lambda}(T) B_{\lambda}(T) d \lambda,
$$

where the absorptive opacity $\alpha_{\lambda}(T)$ is approximated by the one estimated with the previoustemperature [i.e., $\left.\alpha_{\text {abs }}(\boldsymbol{r}, t, \lambda)\right]$. These equations give a new temperature estimate.

The steps (2)-(4) are repeated for a given time step (between $t_{n}$ and $\left.t_{n+1}\right)$ until the temperature converges at all of the meshes simultaneously. Once this happens, the converged temperature is used for the initial guess for the temperature in the next time step, and the photon packets' properties are used as the initial
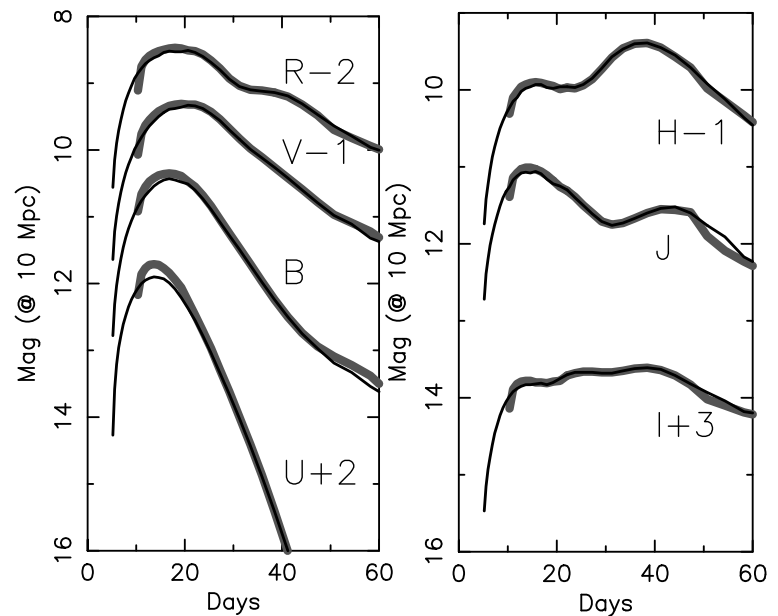

Figure 26. Synthetic multiband light curves of the reference W7 model with different starting times in the simulations. The original simulation (starting at day 10) is shown in gray, and the simulation starting at day 5 is shown in black.
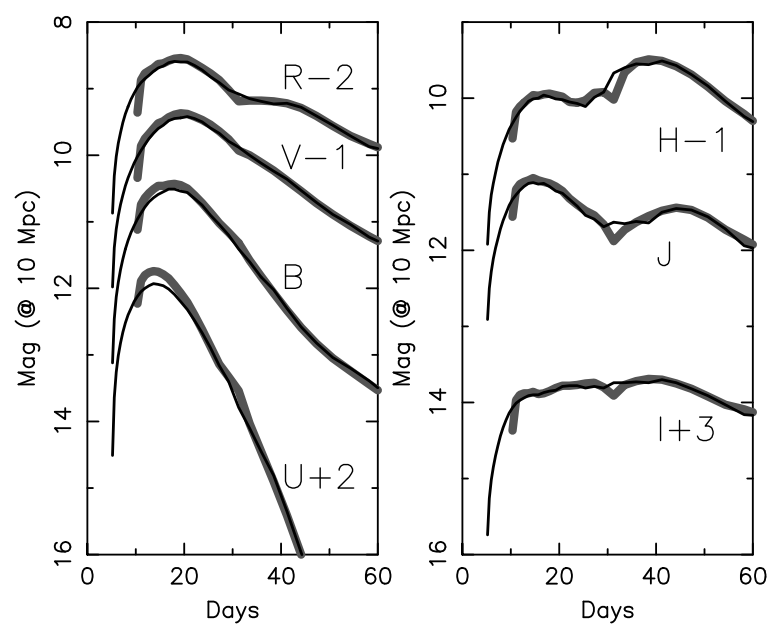

Figure 27. Synthetic multiband light curves of the RGa model with different starting times in the simulations. Shown here are the angle-averaged mean light curves. The original simulation (starting at day 10) is shown in gray, and the simulation starting at day 5 is shown in black.

conditions for the propagation calculations in the next time step $\left(\right.$ at $\left.t_{n+1}\right)$. Then, the procedures (2)-(4) are repeated in the next time step until the temperature convergence. In this way, we proceed with time, following the radiation field and thermal condition in a self-consistent manner.

\section{A.5. Extraction of Synthetic Spectra and Light Curves}

In the MC packet propagation routine, the paths of every photon packet are followed. When the photon packets escape out of the SN ejecta (or the numerical domain), the information is recorded. This provides the escaping radiation flux as a function of the viewing direction, time, and wavelength $\left[f_{\lambda}(\boldsymbol{l}, t)\right]$ (here $l$ is the photon direction vector). From this we extract angledependent spectra as a function of time. The light curves in multiband passes are then extracted by convolving the filter functions to the synthetic spectral sequence. In this paper, we use the Johnson and Kron-Cousins systems for UBVRI and 2MASS systems for the NIR.

Figure 24 shows an example of the synthetic light curves for the W7 model. We find a reasonable agreement between our result and a result obtained by an independent simulation code STELLA (Blinnikov et al. 1998, 2006). While different 

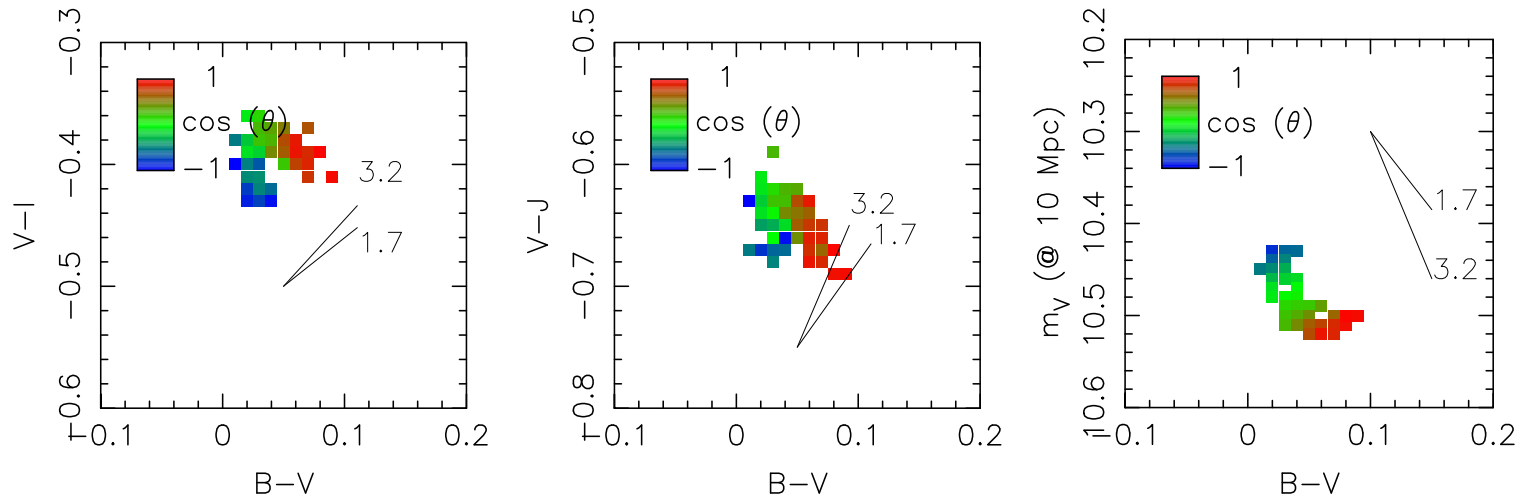

Figure 28. Predicted relations in photometric properties for Model RGa, same as for Figure 12 but with the simulation starting at day 5 .

(A color version of this figure is available in the online journal.)
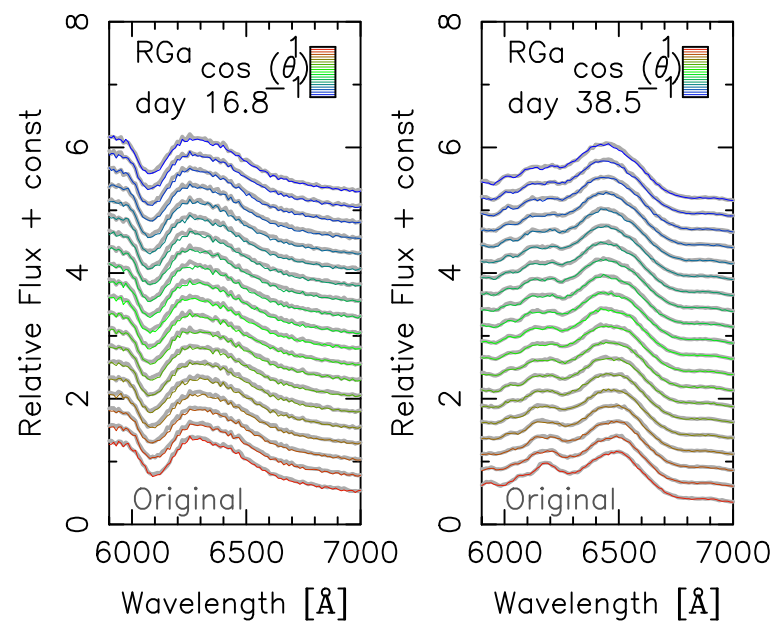

Figure 29. Simulated spectra around Si II 6355 (from the RGa model), the same as for Figure 10 but with the simulation starting at day 5 (color curves). The color coordinates indicate the prediction for different viewing directions (red for $\theta=0$ and blue for $\theta=\pi$ ). In this figure, the spectra for observers at different directions are added with an additional offset ( $\theta=0$ to $\pi$, from bottom to top). The gray curves here are the spectra obtained by the original simulation starting at day 10 .

(A color version of this figure is available in the online journal.)
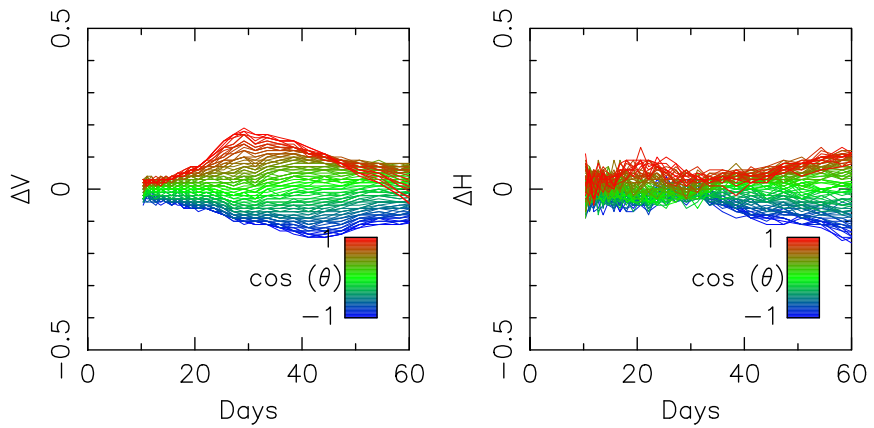

Figure 30. Evolution of the difference between the magnitude for observers at various directions and the angle-averaged magnitude for the RGa model. Shown here are the $V$-band and $H$-band magnitudes.

(A color version of this figure is available in the online journal.)

codes generally agree to reproduce overall behaviors, details are different depending on specific treatments (see, e.g., Kromer $\&$ Sim 2009). Our results are well within these variations and similar to the result of Kasen et al. (2006). We find a reasonable agreement between the W7 model prediction and the Hsiao
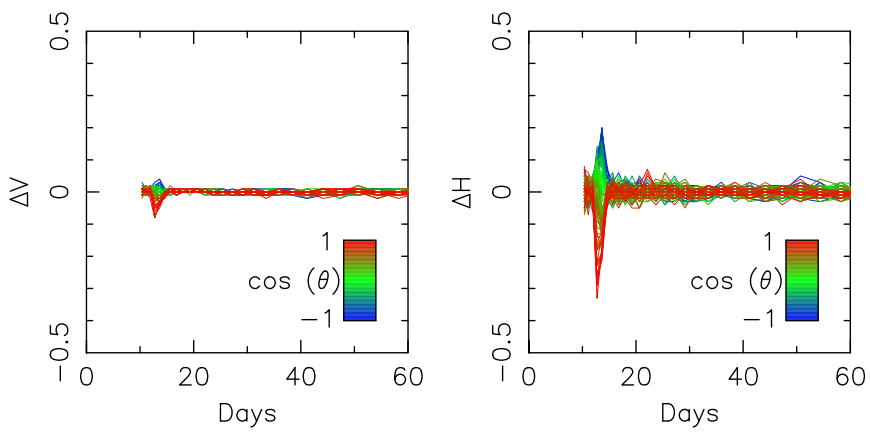

Figure 31. Evolution of the difference between the magnitude for observers at various directions and the angle-averaged magnitude for the reference W7 model but with the instability in the thermal condition artificially introduced at $\theta=165^{\circ}-180^{\circ}$ on day 13.6 (i.e., $\sim 3$ days before the $B$-band maximum). Shown here are the $V$-band and $H$-band magnitudes.

(A color version of this figure is available in the online journal.)

template light curves (Hsiao et al. 2007). The discrepancy is larger in the NIR than in the optical, but this is a general issue in the radiation transfer simulations for $\mathrm{SNe} \mathrm{Ia}$ (see, e.g., Kromer $\& \operatorname{Sim} 2009 ;$ Gall et al. 2012). We note that our reference model (the modified W7) also shows a behavior similar to the original W7, justifying the use of this model as our reference model.

Figure 25 shows a synthetic spectral sequence for the W7 model (red; computed in 1D under the assumption of spherical symmetry), the reference model (blue), and the Hsiao template spectra (black) (Hsiao et al. 2007). We see a reasonable agreement between the W7 model and the Hsiao templates. There are deviations especially in the later phases, while the model does predict spectral features at the correct wavelengths, the strengths of the features can be different from the observed templates. This is suggested to be caused by NLTE effects (e.g., Kromer \& Sim 2009), and this is a generic issue for spectrum synthesis in SNe (see, e.g., Sim et al. 2013, for 3D delayeddetonation models). Our reference model shows a larger flux than the W7 and the Hsiao templates due to the large amount of ${ }^{56} \mathrm{Ni}$, but otherwise the predicted features are very similar to the W7 model. Therefore, using this model as our reference is justified. We also show the same W7 models but mapped onto the 2D grids and computed in 2D, under the assumption of axisymmetry (but no symmetry with respect to the equatorial direction). We see a perfect match between the 1D and 2D calculations, proving that our $2 \mathrm{D}$ radiation transfer scheme does not introduce any errors in the transfer simulation. 

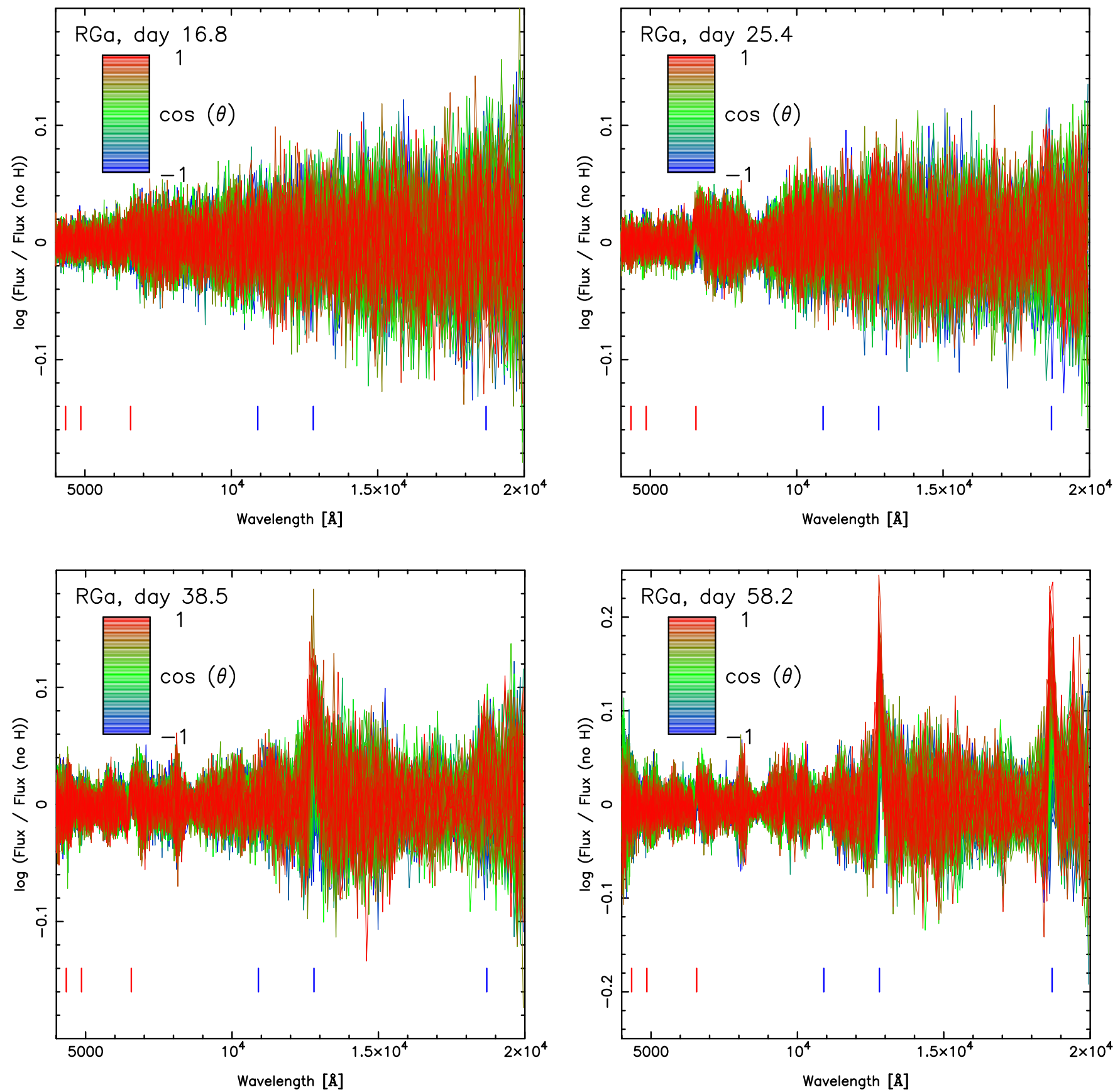

Figure 32. Ratio of the spectral flux with and without hydrogen lines in Model RGa. This is the same as in Figure 15, except that the hydrogen bound-bound transitions are treated as full resonance lines (i.e., $\epsilon=0$ ).

(A color version of this figure is available in the online journal.)

\section{APPENDIX B}

\section{ROBUSTNESS OF THE PREDICTED MAXIMUM-PHASE BEHAVIORS}

In this paper, we deal with the diversity in the magnitudes and colors around the $B$-band maximum at the $0.1 \mathrm{mag}$ level. In this section, we show that our simulations are accurate to this level to claim the diversity arising from the different viewing directions. In particular, we address the following two points: (1) whether the relatively late starting time in our simulations (10 days) affects the claimed behaviors, and (2) whether the predicted diversities and correlations are not affected by possible numerical instabilities.
Figure 26 shows the multiband light curves of the reference W7 model from the simulation starting at day 5 , as compared to our standard run starting at day 10 . The same but for the RGa model is shown in Figure 27. It is seen that the two calculations with different starting times converge quickly toward the $B$-band maximum date in both models. A substantial difference is seen in the $U$-band light curve around its peak date (before the $B$-band peak), but in the other bands the difference is small. This test also shows that (late-phase) kinks seen in the original calculations, especially in the $J$ band (i.e., $\sim 50$ days for the reference model and $\sim 30$ days in the RGa model), are numerical artifacts. The fact that these kinks appear much later than the $B$-band peak where the treatment of the starting time should be unimportant 

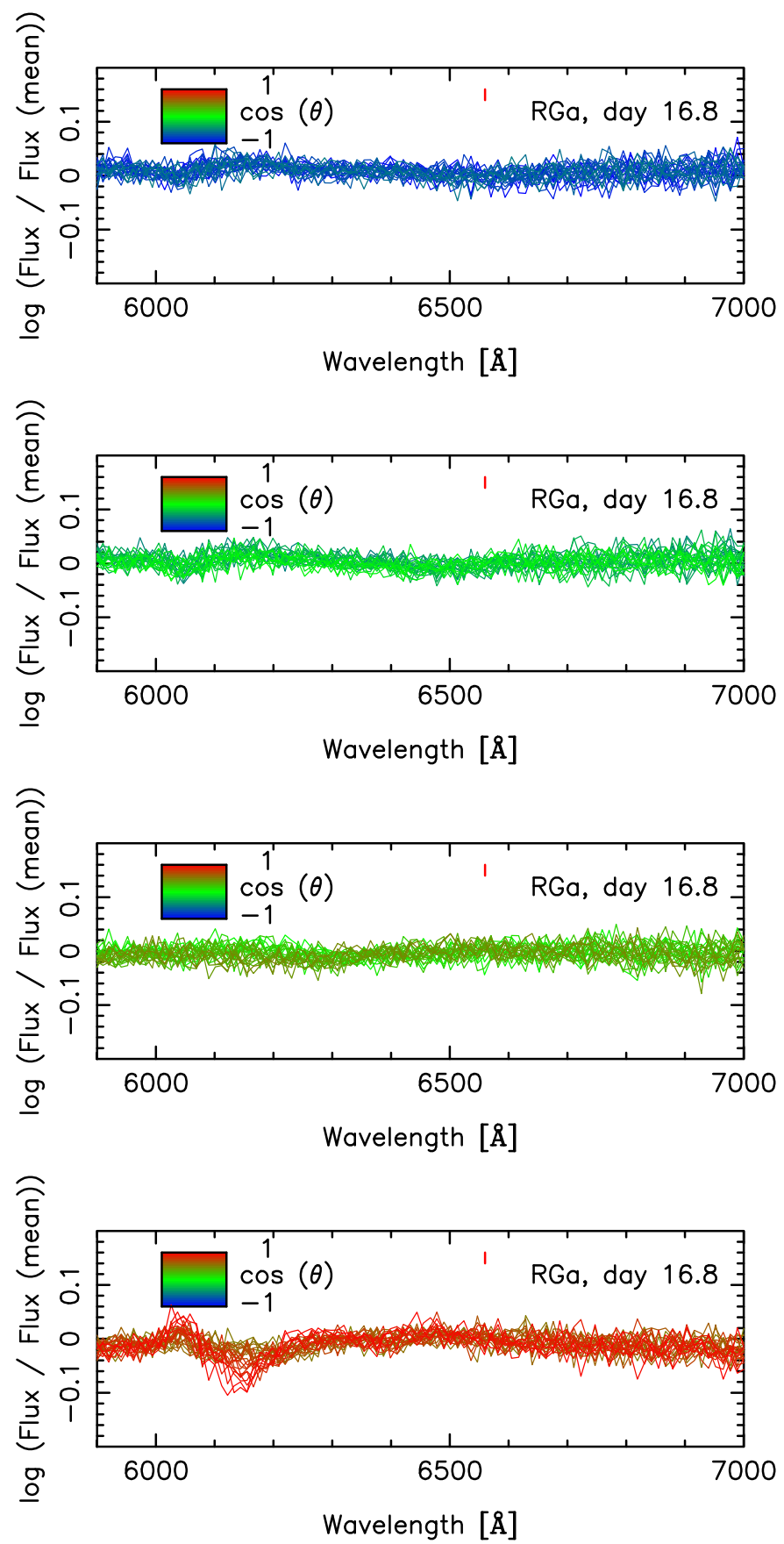

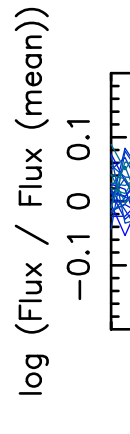

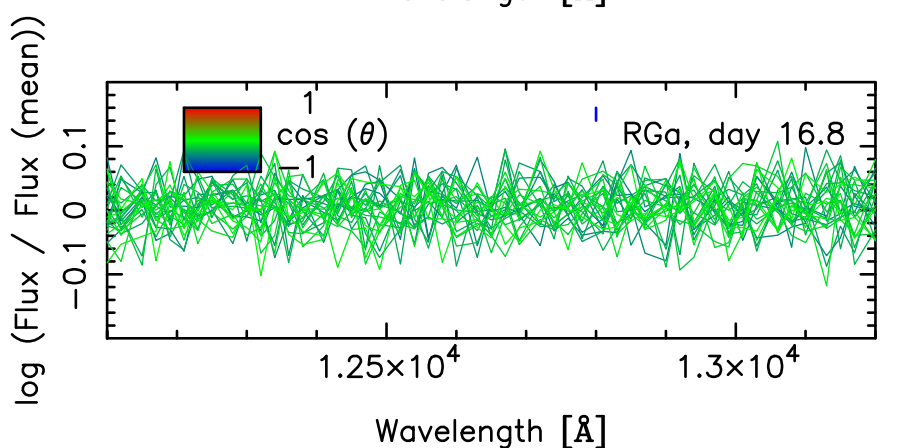
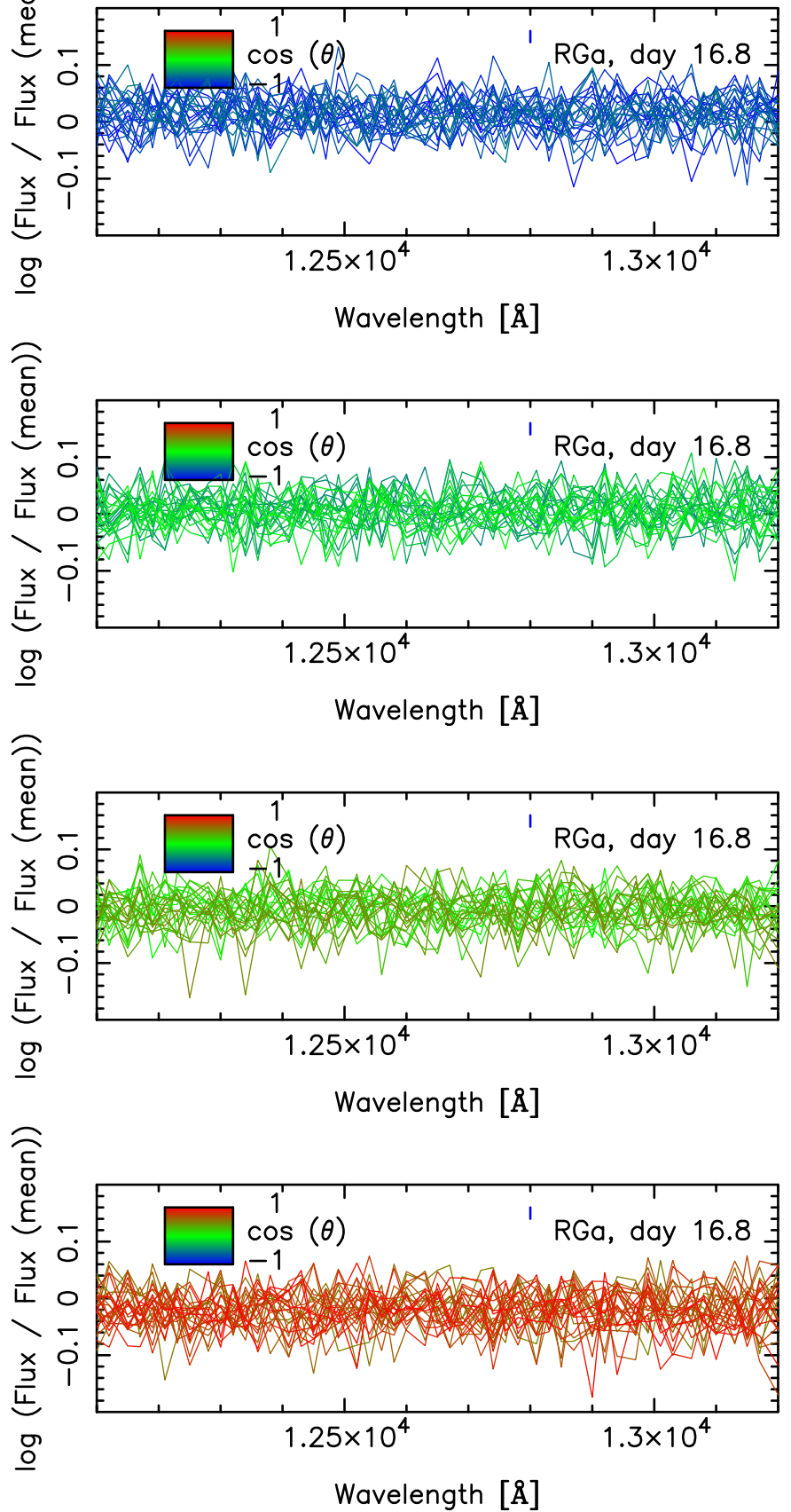

Wavelength $[\AA]$

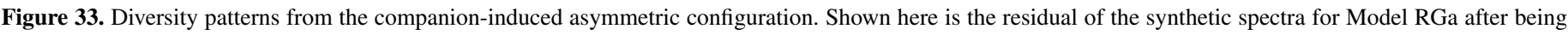

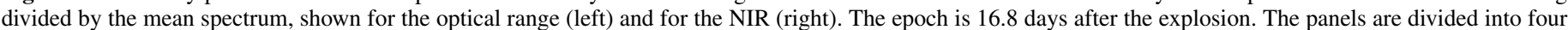

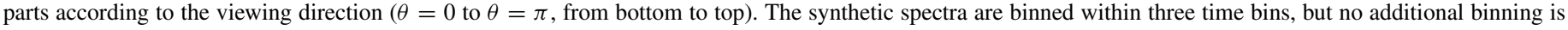
performed in the wavelength and viewing angle directions.

(A color version of this figure is available in the online journal.)

indicates that this late-phase stability can be sensitive to small variations in the thermal conditions, but fortunately it seems that this unstable behavior does not appear around the maximum phase (see also below).

Figure 28 shows the variations in the colors and the $V$-band magnitude and their relations to the $B-V$ color obtained for Model RGa for the simulation starting at day 5 . This should be compared to Figure 12, where the simulation is started at day 10. Although a small offset in the absolute scale is seen for the $B-V$ and $V-I$ colors and the $V$-band magnitude at the level of $0.05 \mathrm{mag}$, the trend as a function of different viewing directions and the amount of the resulting diversity are consistent with the original simulation.

The spectral features are even less sensitive to the starting time of the simulation. Figure 29 compares the spectra around Si II 6355 in both simulations. It is seen that the results are almost identical, and therefore the relations involving the Si velocity should also be unaffected by the treatment of the starting time.

We note that while the absolute magnitude can be affected by the treatment of the starting time (which turns out to be $0.05 \mathrm{mag}$ level), the behavior in the colors and magnitudes arising from different viewing directions should be much less 

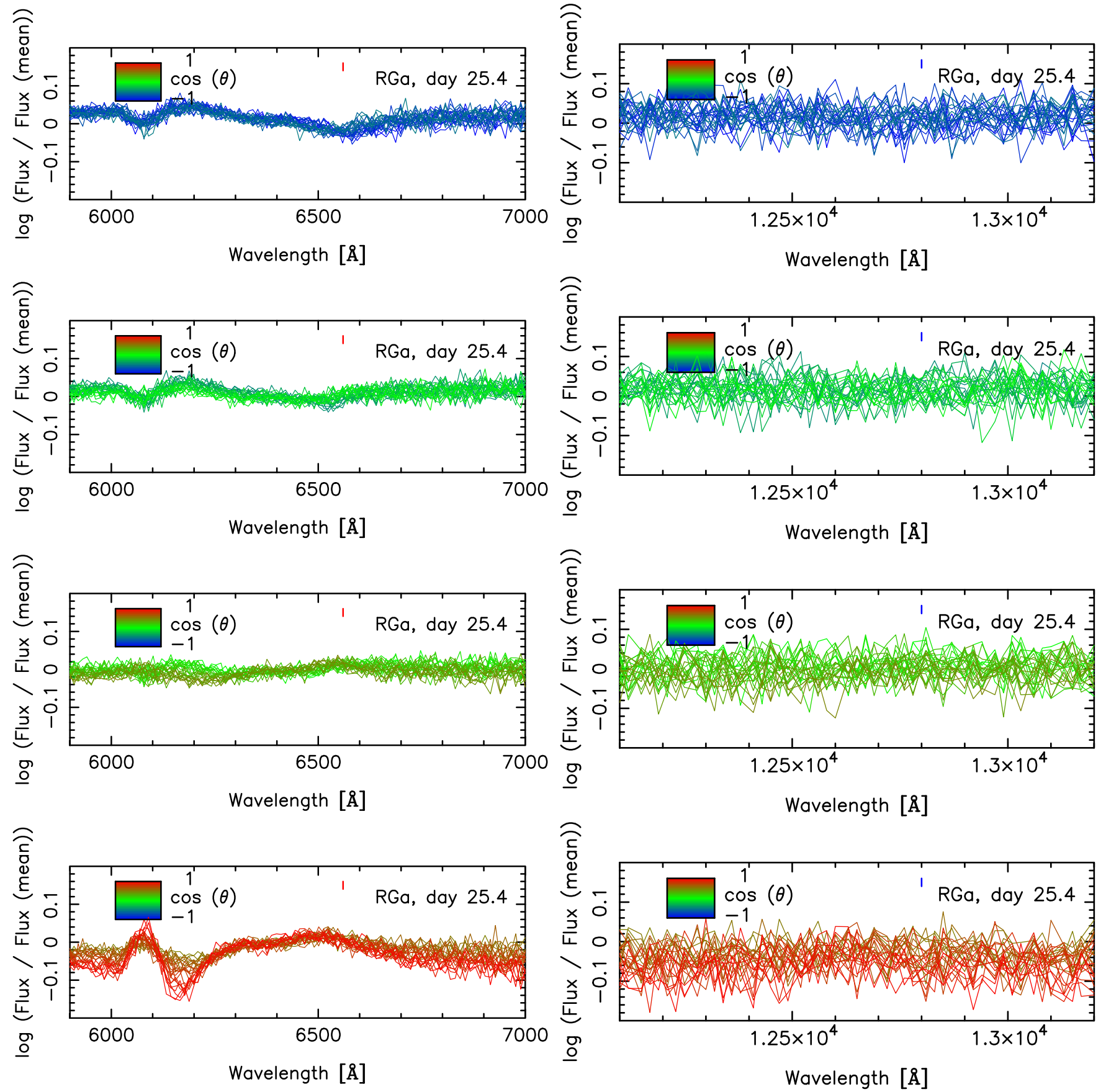

Figure 34. Diversity patterns from the companion-induced asymmetric configuration, the same as in Figure 33 . The epoch is 25.4 days after the explosion.

(A color version of this figure is available in the online journal.)

sensitive here. The spectral features are expected to be even less sensitive. These are confirmed by the test simulation, justifying our claims regarding the correlation and diversities arising from different viewing directions.

Another issue is whether the trend and diversity arising from the different viewing directions around the $B$-band maximum are affected by numerical instability similar to that seen in the $J$-band light curves in the later phase. First, the investigation of the sensitivity to the starting time suggests that such an instability is less likely to take place in the earlier phase than in the later phase. The different thermal conditions due to the different starting times may mimic numerical instability in the thermal condition, but we see that the thermal condition quickly converges before the $B$-band maximum. This is expected because the radiation-matter coupling is quite strong in the early phase, which should suppress the numerical instability quickly (note that the iteration in the thermal condition is performed in every time step under the assumption of radiative equilibrium). Next, for such an instability to affect the viewingangle dependence, the instability itself should create a strong angle-dependent effect, which should be seen as an angledependent sudden rise and fall in the multiband light curves. Even for the possible instability found in the later phases (in the $J$ band), this effect does not show strong angle dependence. Therefore, even if a similar numerical instability would take place in the earlier phase, it is unlikely that such a putative effect should affect the angle-dependent effects that we claim in the present paper. 

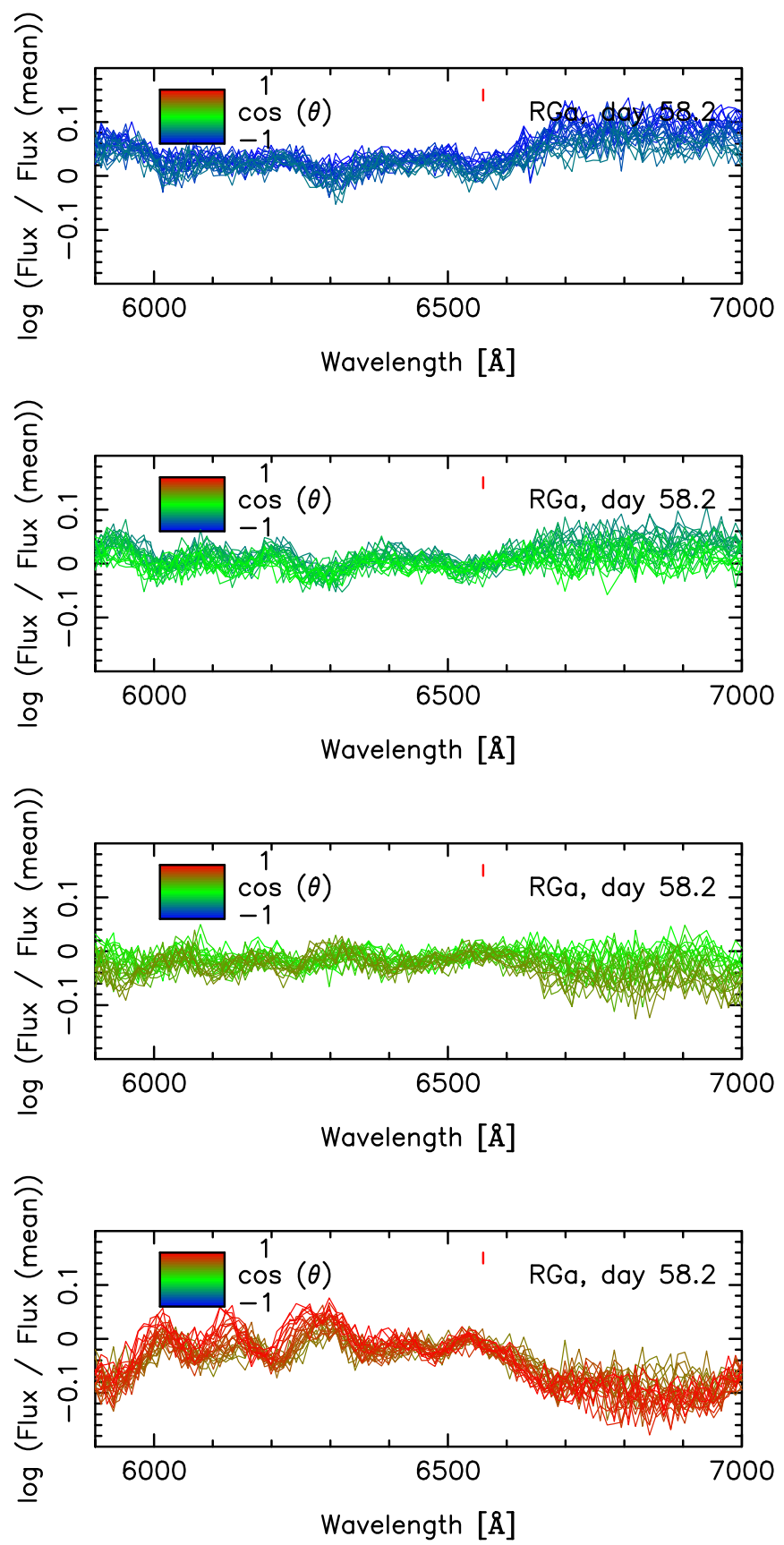

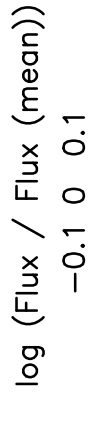

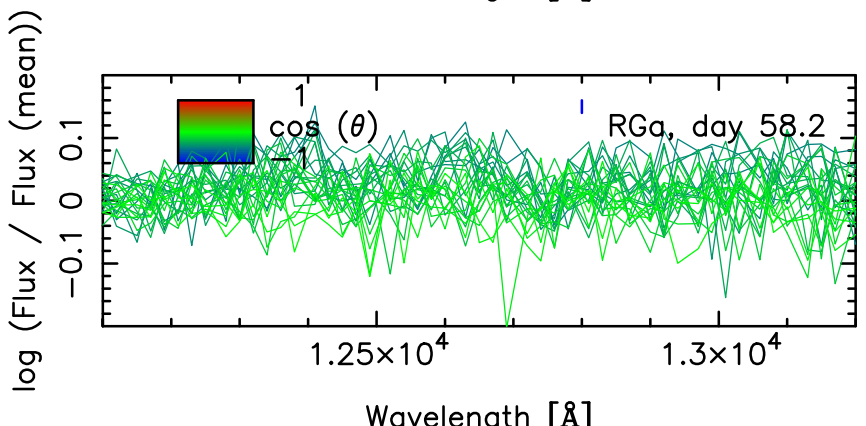
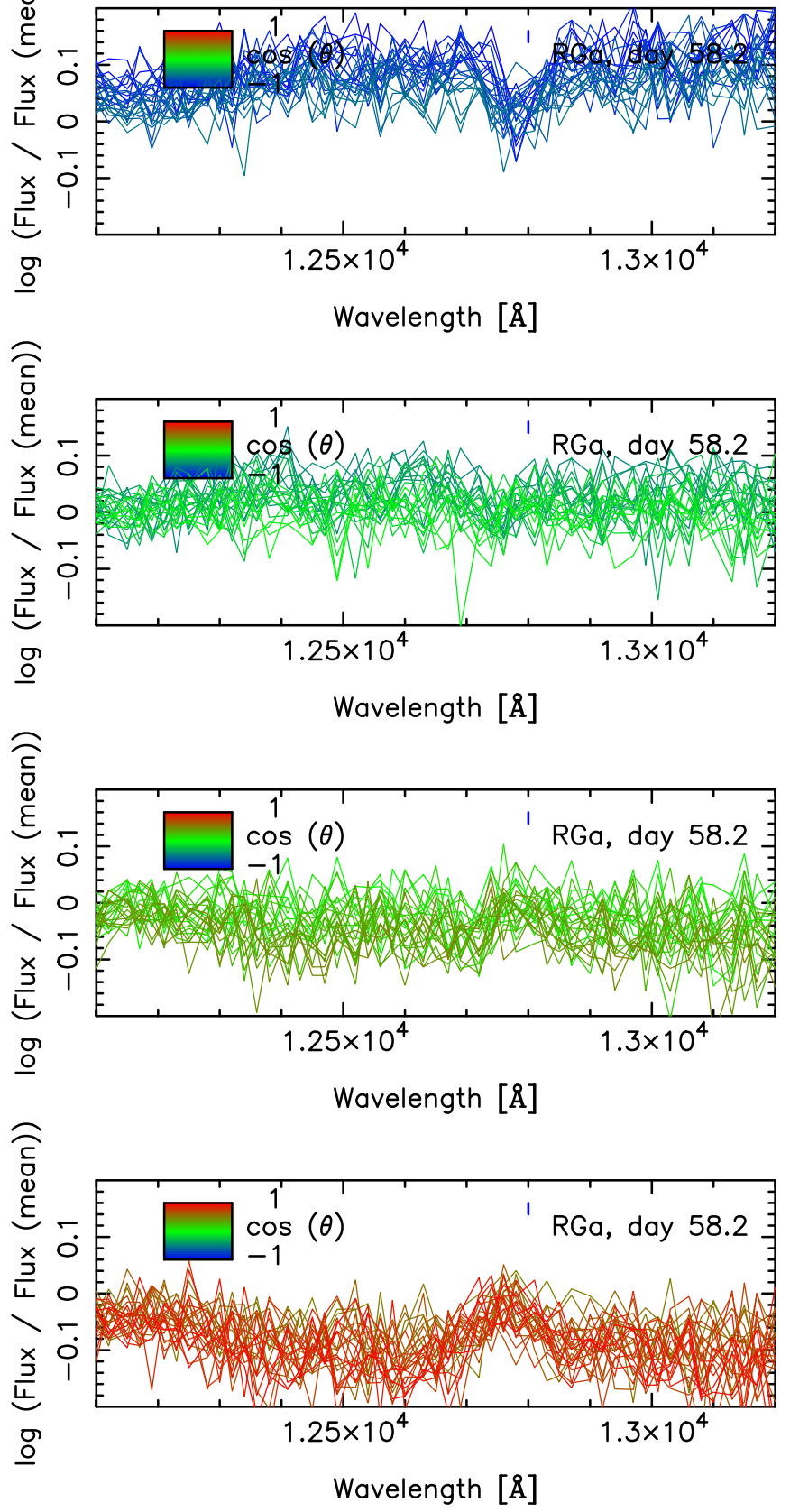

Wavelength $[\AA]$

Wavelength $[\AA]$

Figure 35. Diversity patterns from the companion-induced asymmetric configuration, the same as in Figure 33 . The epoch is 58.2 days after the explosion.

(A color version of this figure is available in the online journal.)

To support these arguments, we have performed a test calculation based on the reference W7 model. Here, on day 13.6 (i.e., $\sim 3$ days before the $B$-band maximum), we artificially reduce the temperature in the ejecta only within $\theta=165-180$ degrees to $40 \%$ of the real converged temperature. As such, if the instability does not fade away, this should create artificially introduced viewing-angle dependence for the expected observables. The setup here is chosen, following trial and error, so that the inserted artificial effect on the viewing angle variation exceeds the variation seen in the RGa model.

Figure 30 shows the variation of the $V$-band and $H$-band magnitudes in the RGa model due to different viewing directions, as compared to the angle-averaged mean magnitude in each band. The evolution for a given $\theta$ is quite continuous, and we do not see any sudden change in the viewing angle dependence, which would announce any possible numerical instabilities. This should be compared with Figure 31, which shows the same quantities for the reference W7 model where the numerical instability is artificially inserted in the cone (in the direction of $\theta=165-180$ degrees). We see here a sudden broadening of the angle dependence, especially in the $H$ band. Such a behavior is not seen in the RGa model, suggesting that any instability that creates numerically introduced viewing dependence larger than the real physical behavior is not present in the simulation for the RGa model.

Furthermore, the artificially introduced variation for different viewing directions quickly fades away, and the remaining variation (due to the MC noise) becomes much smaller than 
the variations seen in the RGa model. This further supports the idea that the viewing angle dependence around the $B$-band maximum is not affected by any instabilities and the MC noise in our simulations is sufficiently small. In sum, we conclude that our claim on the viewing-angle dependence on the $B$-band maximum observables (e.g., Figures 12 and 14) is not due to numerical artifacts.

\section{APPENDIX C}

\section{TREATMENT OF HYDROGEN LINES}

Because we have adopted the expansion opacity formalism and the two-level atom approximation, a question is whether the predicted fluxes of the hydrogen features can be significantly affected by these assumptions (Section 6). Previous studies suggest that it would not introduce a large difference (Baron et al. 1996; Dessart \& Hiller 2008; Kasen \& Woosley 2009). Here we evaluate a possible effect of this for the situation we investigate.

We are interested to see whether the hydrogen line fluxes are much affected by the treatment of the bound-bound transitions for a given thermal condition. Thus, we have computed the model spectra for Model RGa under an extreme assumption on the hydrogen line formation, namely pure scattering (full resonance) line transitions within our formalism $(\epsilon=0)$. To do this, we have taken the thermal condition computed from the original calculations $(\epsilon=0.3)$ so that we can purely pick up the effect of $\epsilon$ on the line formation. Then the model calculations with and without hydrogen (for the latter we artificially set the cross sections of hydrogen to zero) are performed, and the ratio is investigated as in Section 5. Figure 32 shows the result. We thereby confirmed that this does not affect the detectability of the hydrogen lines. Because we expect that the scatteringdominated atmosphere is an extreme condition, we believe that the hydrogen line formation we find in this paper would not be much affected by the treatment of hydrogen line transitions.

\section{APPENDIX D \\ DIVERSITY PATTERNS FROM THE COMPANION-INDUCED ASYMMETRY}

Irrespective of the hydrogen content in the expanding SN ejecta, the companion-induced asymmetry can create characteristic diversity patterns as a function of the wavelength. This can in principle be tested by observations, by studying a diversity seen in spectra of individual $\mathrm{SNe}$ as compared to a reference/ template spectrum. The reference spectrum should be created as a mean of the spectra of $\mathrm{SNe}$ in a specific subgroup (or all $\mathrm{SNe}$ Ia), and the comparison can be performed between an individual and the reference spectra. It will allow us to see a possible diversity of individual SNe from the average behavior of the group. The diversity pattern predicted from the companion-induced asymmetry is different for different viewing directions, and it evolves with time. A specific example of the expected diversity pattern is shown in Figures 21 and 22 for an epoch of 38.5 days after the explosion. In Figures 33-35, we provide the model predictions for different epochs.

\section{REFERENCES}

Aldering, G., Antilogus, P., Bailey, S., et al. 2006, ApJ, 650, 510 Baron, E., Höflich, P. H., Nugent, P., \& Branch, D. 1996, MNRAS, 283, 297 Bedin, L. R., Ruiz-Lapuente, P., González Hernández, J. I., et al. 2014, MNRAS, 439,354
Benetti, S., Cappellaro, E., Mazzali, P. A., et al. 2005, ApJ, 623, 1011

Benetti, S., Meikle, P., Stehle, M., et al. 2004, MNRAS, 348, 261

Blinnikov, S. I., Eastman, R., Bartunov, O. S., Popolitov, V. A., \& Woosley, S. E. 1998, ApJ, 496, 454

Blinnikov, S. I., Röpke, F. K., Sorokina, E. I., et al. 2006, A\&A, 453, 229

Blondin, S., Matheson, T., Kirshner, R. P., et al. 2012, AJ, 143, 126

Branch, D., Dang, L. C., Hall, N., et al. 2006, PASP, 118, 560

Castor, J. I. 1972, ApJ, 178, 779

Dessart, L., \& Hiller, D. J. 2008, MNRAS, 383, 57

Dilday, B., Howell, D. A., Cenko, S. B., et al. 2012, Sci, 337, 942

Eastman, R. G., \& Pinto, P. A. 1993, ApJ, 412, 731

Folatelli, G., Phillips, M. M., Burns, C. R., et al. 2010, AJ, 139, 120

Foley, R. J., \& Kasen, D. 2011, ApJ, 729, 55

Gall, E. E. E., Taubenberger, S., Kromer, M., et al. 2012, MNRAS, 427, 994

González Hernánedz, J. I., Ruiz-Lapuente, P., Tabernero, H. M., et al. 2012, Natur, 489, 533

Hachisu, I., Kato, M., \& Nomoto, K. 1999, ApJ, 522, 487

Hachisu, I., Kato, M., \& Nomoto, K. 2012, ApJL, 756, L4

Hamuy, M., Maza, J., Pinto, P. A., et al. 2002, ApJ, 124, 417

Hamuy, M., Phillips, M. M., Suntzeff, N. B., et al. 2003, Natur, 424, 651

Hsiao, E. Y., Conley, A., Howell, D. A., et al. 2007, ApJ, 663, 1187

Hyden, B. T., Garnavich, P. M., Kasen, D., et al. 2010, ApJ, 722, 1691

Iben, I., Jr., \& Tutukov, A. V. 1984, ApJ, 284, 719

Ihara, Y., Ozaki, J., Doi, M., et al. 2007, PASJ, 59, 811

Karp, A. H., Lasher, G., Chan, K. L., \& Salpeter, E. E. 1977, ApJ, 214, 161

Kasen, D. 2010, ApJ, 708, 1025

Kasen, D., Nugent, P., Thomas, R. C., \& Wang, L. 2004, ApJ, 610, 876

Kasen, D., Thomas, R. C., \& Nugent, P. 2006, ApJ, 651, 366

Kasen, D., \& Woosley, S. E. 2009, ApJ, 703, 2205

Kerzendorf, W., Schmidt, B. P., Asplund, M., et al. 2009, ApJ, 701, 1665

Kromer, M., \& Sim, S. A. 2009, MNRAS, 398, 1809

Kurucz, R., \& Bell, B. 1995, Atomic Line Data, Kurucz CD-ROM 23 (Cambridge: $\mathrm{SAO})$

Kutsuna, M. 2013, PhD thesis, Univ. Tokyo

Kutsuna, M., \& Shigeyama, T. 2013, ApJ, submitted

Lentz, E. J., Baron, E., Hauschuldt, P. H., \& Branch, D. 2002, ApJ, 580, 374

Leonard, D. C. 2007, ApJ, 670, 1275

Li, W., Bloom, J. S., Podsiadlowski, P., et al. 2011, Natur, 480, 348

Liu, Z.-W., Pakmor, R., Röpke, F. K., et al. 2012, A\&A, 548, 2

Liu, Z.-W., Pakmor, R., Seitenzahl, I. R., et al. 2013, ApJ, 774, 37

Lucy, L. B. 2005, A\&A, 429, 19

Lundqvist, P., Mattila, S., Sollerman, J., et al. 2013, MNRAS, 435, 329

Maeda, K. 2006, ApJ, 644, 385

Maeda, K., Benetti, S., Stritzinger, M., et al. 2010, Natur, 466, 82

Maeda, K., Leloudas, G., Taubenberger, S., et al. 2011, MNRAS, 413, 3075

Maeda, K., Mazzali, P. A., Deng, J., et al. 2003, ApJ, 593, 931

Marietta, E., Burrows, A., \& Fryxell, B. 2000, ApJS, 128, 615

Marion, G. H., Höflich, P., Gerardy, C. L., et al. 2009, AJ, 138, 727

Marion, G. H., Höflich, P., Wheeler, J. C., et al. 2006, ApJ, 645, 1392

Mattila, S., Lundqvist, P., Sollerman, J., et al. 2005, A\&A, 443, 649

Nomoto, K. 1982, ApJ, 253, 798

Nomoto, K., Thielemann, F.-K., \& Yokoi, K. 1984, ApJ, 286, 644

Nugent, P., Phillips, M. M., Baron, E., Branch, D., \& Hauschildt, P. 1995, ApJL, 455, L147

Pakmor, R., Kromer, M., Röpke, F. K., et al. 2010, Natur, 463, 61

Pakmor, R., Röpke, F. K., Weiss, A., \& Hillebrandt, W. 2008, A\&A, 489, 943

Permutter, S., Aldering, G., Goldhaber, G., et al. 1999, ApJ, 517, 565

Phillips, M. M., Lira, P., Suntzeff, N. B., et al. 1999, AJ, 118, 1766

Riess, A. G., Filippenko, A. V., Challis, P., et al. 1998, AJ, 116, 1009

Ruiz-Lapuente, P., Comeron, F., Méndez, J., et al. 2004, Natur, 431, 1069

Saio, H., \& Nomoto, K. 1985, A\&A, 150, L21

Schaefer, B. E., \& Pagnotta, A. 2012, Natur, 481, 164

Shappee, B. J., Stanek, K. Z., Pogge, R. Q., \& Garnavich, P. M. 2013, ApJL, 762, L5

Silverman, J. M., Kong, S. S., \& Filippemko, A. V. 2012, MNRAS, 425, 1819

Sim, S. A., Seitenzahl, I. R., Kromer, M., et al. 2013, MNRAS, 436, 333

Stanishev, V., Goobar, A., Benetti, S., et al. 2007, A\&A, 469, 645

Verner, D. A., Ferland, G. J., \& Korista, K. T. 1996, AJ, 465, 487

Verner, D. A., \& Yakovlev, D. G. 1995, A\&AS, 109, 125

Wang, B., Chen, X., Meng, X., \& Han, Z. 2009, ApJ, 701, 1540

Wang, X., Wang, L., Filippenko, A. V., Zhang, T., \& Zhao, X. 2013, Sci, 340,170

Webbink, R. F. 1984, ApJ, 277, 355

Wheeler, J. C. 2012, ApJ, 758, 123

Whelan, J., \& Iben, I., Jr. 1973, ApJ, 186, 1007

Yaron, O, \& Gal-Yan, A. 2012, PASP, 124, 668

Yoon, S.-C., Podsiadlowski, Ph., \& Rosswog, S. 2007, MNRAS, 380, 933 